\title{
Surface electrical stimulation of the neck for oropharyngeal dysphagia in Parkinson's disease : therapeutic aspects and reliability of measurement
}

Citation for published version (APA):

Baijens, L. W. J. (2014). Surface electrical stimulation of the neck for oropharyngeal dysphagia in Parkinson's disease : therapeutic aspects and reliability of measurement. [Doctoral Thesis, Maastricht University]. Datawyse / Universitaire Pers Maastricht. https://doi.org/10.26481/dis.20140214lb

Document status and date:

Published: 01/01/2014

DOI:

10.26481/dis.20140214lb

Document Version:

Publisher's PDF, also known as Version of record

\section{Please check the document version of this publication:}

- A submitted manuscript is the version of the article upon submission and before peer-review. There can be important differences between the submitted version and the official published version of record. People interested in the research are advised to contact the author for the final version of the publication, or visit the DOI to the publisher's website.

- The final author version and the galley proof are versions of the publication after peer review.

- The final published version features the final layout of the paper including the volume, issue and page numbers.

Link to publication

\footnotetext{
General rights rights.

- You may freely distribute the URL identifying the publication in the public portal. please follow below link for the End User Agreement:

www.umlib.nl/taverne-license

Take down policy

If you believe that this document breaches copyright please contact us at:

repository@maastrichtuniversity.nl

providing details and we will investigate your claim.
}

Copyright and moral rights for the publications made accessible in the public portal are retained by the authors and/or other copyright owners and it is a condition of accessing publications that users recognise and abide by the legal requirements associated with these

- Users may download and print one copy of any publication from the public portal for the purpose of private study or research.

- You may not further distribute the material or use it for any profit-making activity or commercial gain

If the publication is distributed under the terms of Article $25 \mathrm{fa}$ of the Dutch Copyright Act, indicated by the "Taverne" license above, 
Surface electrical stimulation of the neck for oropharyngeal dysphagia in Parkinson's disease: therapeutic aspects and reliability of measurement 
(C) Laura Baijens, Maastricht 2014

Layout: Tiny Wouters

Production: Datawyse | Universitaire Pers Maastricht

ISBN: 9789461593023

Financial support for the publication of this thesis was kindly provided by:

Atos Medical BV, NestléHealthScience, Nutricia Advanced Medical Nutrition, Pentax Medical. 


\title{
Surface electrical stimulation of the neck for oropharyngeal dysphagia in Parkinson's disease: therapeutic aspects and reliability of measurement
}

\author{
PROEFSCHRIFT \\ ter verkrijging van de graad van doctor aan de Universiteit Maastricht, \\ op gezag van de Rector Magnificus, Prof. Dr. L.L.G. Soete \\ volgens het besluit van het College van Decanen, \\ in het openbaar te verdedigen \\ op vrijdag 14 februari 2014 om 12.00 uur \\ door \\ Laura Wilhelmine Jeanette Baijens \\ Geboren op 21 juni 1972 te Helmond
}

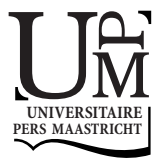




\section{Promotor}

Prof. dr. B. Kremer

\section{Copromotor}

Dr. R. Speyer

\section{Beoordelingscommissie}

Prof. dr. R.J. van Oostenbrugge (voorzitter)

Dr. A.F.G. Leentjens

Prof. dr. A.A.M. Masclee

Prof. dr. M.W.M. van den Brekel (Universiteit van Amsterdam, Antoni van Leeuwenhoek Nederlands Kanker Instituut) 


\section{Contents}

$\begin{array}{lll}\text { Chapter } 1 & \text { General introduction and outline of the thesis. } & 7\end{array}$

Chapter 2 Effects of therapy for dysphagia in Parkinson's disease: 17

systematic review.

Dysphagia. 2009;24:91-102.

Chapter 3 Swallowing in Parkinson patients versus healthy controls:

reliability of measurements in videofluoroscopy.

Gastroenterol Res Pract. Epub 2011 Oct 3.

Chapter 4 The effect of surface electrical stimulation on swallowing in dysphagic Parkinson patients.

Dysphagia. 2012;27:528-537.

Chapter 5 Surface electrical stimulation in dysphagic Parkinson patients:

a randomized clinical trial.

The laryngoscope. 2013;123:E38-44.

Chapter 6 FEES protocol derived estimates of sensitivity to aspiration in dysphagic patients: the number of swallow trials versus aspiration risk.

Submitted. Under revision.

Chapter 7 Summary, general discussion, and future perspectives.

Samenvatting

Dankwoord

Curriculum vitae

List of publication 



\section{Chapter 1}

General introduction
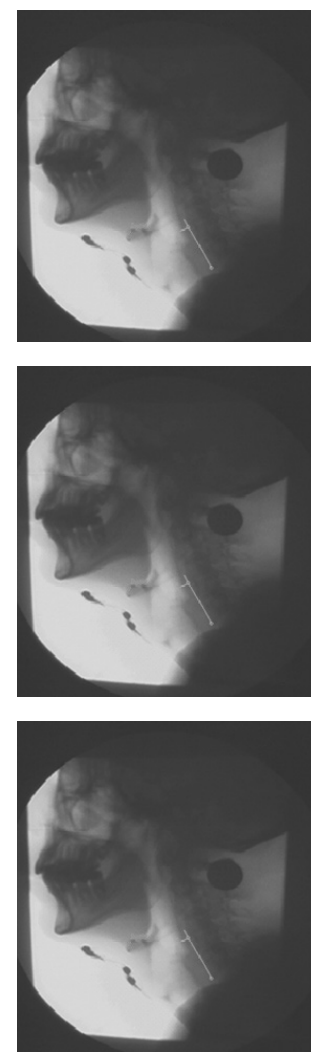
Chapter 1 


\section{Idiopathic Parkinson's disease}

"An essay on the shaking palsy" was published in 1817 by a British medical doctor named James Parkinson, the first to describe the muscle weakness and tremor associated with idiopathic Parkinson's disease (IPD) (Figure 1.1) $)^{1}$. Nowadays its prevalence ranges from 102 to 190 per 100,000 population in western countries ${ }^{2}$ and its annual incidence from 4.9 to 26 per $100,000^{2}$. IPD is characterized by the adult-onset degenerative loss of neuronal cells, specifically the dopaminergic neurons of the midbrain in the substantia nigra (Figure 1.2). While the exact etiology remains unknown, IPD may result from a complex interaction between environmental toxic factors, genetic predisposition, and aging. Neuroimaging techniques can assist in the diagnosis, which is made on the basis of clinical symptoms ${ }^{3}$. Motor as well as nonmotor symptoms become more prominent as the disease progresses. Motor abnormalities such as tremor at rest, rigidity, slowness of voluntary movement, and postural instability can appear.

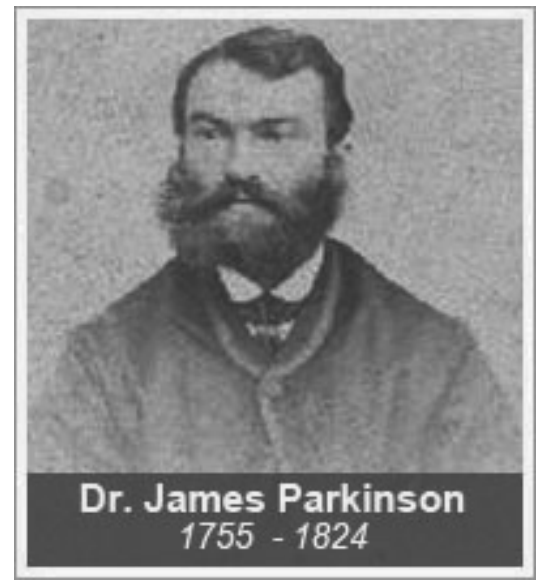

Figure 1.1

Several nondopaminergic neurotransmitter systems are involved in IPD too. Because depression, behavioral and sleep disturbances, dysarthria, freezing while in the "on" motor phase, postural instability, cognitive impairment, and autonomic dysfunction may not find their pathophysiology in the damage to the dopaminergic system, these symptoms usually fail to improve with Levodopa (L-dopa) therapy. Dysfunction of nondopaminergic systems often has more impact on the quality of life of these patients than primary parkinsonian motor dysfunction ${ }^{3}$.

The international gold standard of clinical rating scales for IPD is the Unified Parkinson's Disease Rating Scale (UPDRS) to assess impairment and disability. Another clinical rating scale, the Hoehn and Yahr scale, is commonly used to describe how the symptoms 
progress. The latter scale divides the severity of IPD into five stages ${ }^{4}$ : stage 1 , only one side of the body is affected, usually with minimal or no functional impairment; stage 2, both sides are affected, but posture and balance remain normal; stage 3 , both sides are affected, with mild imbalance when standing or walking; stage 4, both sides are affected, with disabling instability while standing or walking, i.e., the person requires substantial help and cannot live alone; and stage 5, severe, fully developed disease, i.e., the person is often cachectic and restricted to bed or a wheelchair unless aided ${ }^{4}$. In the early stages, the patient - or rather, the brain - can often compensate for the swallowing disorder to some extent, as described in the following section. But in an advanced stage, almost all patients will suffer from severe oropharyngeal dysphagia ${ }^{3}$. Considerable progress has been made since the introduction of the first dopamine-replacing drug, L-dopa, in treating parkinsonian motor features. Although a wide range of dopaminergic treatments are available, they often lead to problems at advanced disease stages.

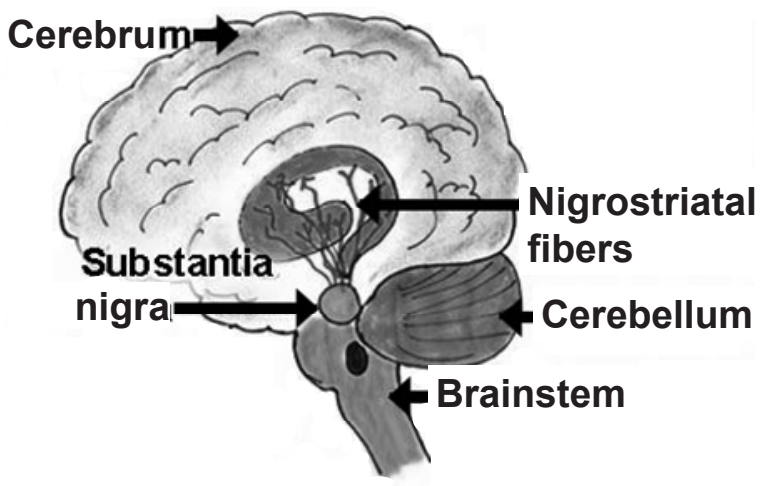

Figure 1.2 Schematic anatomy of the human brain.

\section{Oropharyngeal dysphagia and IPD}

Attention to the diagnosis and treatment of oropharyngeal dysphagia has grown over the past decades because of the rising number of patients suffering from IPD ${ }^{2,3}$. Up to $80 \%$ of them will have oropharyngeal dysphagia, albeit in a very mild form, during the early stages; in the advanced stages, almost $95 \%$ will have it ${ }^{5,6,7}$. Quality of life depends on, among other things, the ability to eat varied and tasty meals $\mathrm{s}^{8,9}$. However, oropharyngeal dysphagia often reduces one's capacity for oral intake of various foods. As it progresses, it may call for bolus modification and could result in an unbalanced diet, malnutrition, and loss of appetite ${ }^{10,11,12,13,14}$. In the early stages, the swallowing function is relatively well preserved $^{5,15}$, though this finding is refuted by a few authors who find no correlation between the presence or severity of dysphagia and overall disease severity ${ }^{16}$. As patients 
often use compensatory mechanisms in the early stages of IPD, the swallowing disorder may be benign ${ }^{5}$, though complications like aspiration may occur, especially in an advanced stage $^{6,16}$. Mortality due to aspiration pneumonia has been studied in this patient population ${ }^{17,18}$, but the pathophysiology of the underlying oropharyngeal dysphagia is poorly understood. Dysfunction of the swallowing central pattern generator (brainstem) and degeneration of the substantia nigra seem to be important causes, and disturbance of nondopaminergic neural networks may be a major contributing factor. Unlike other motor-related symptoms, oropharyngeal dysphagia responds to dopaminergic treatment only in a small proportion of patients ${ }^{3,19,20,21}$. Its persistence in the "on" motor phase is likely to result from disturbances in the medullary swallowing central pattern generator ${ }^{22}$. Moreover, cortical areas seem to modulate swallowing function in IPD. Adaptive cerebral changes in swallowing apparently compensate for deficient motor pathways ${ }^{23}$. Recruitment of better-preserved parallel motor loops driven by sensory afferent input is said to maintain swallowing function until progressing neurodegeneration exceeds the means of this adaptive strategy. At that point, oropharyngeal dysphagia manifests itself ${ }^{23}$. Various motor disorders of IPD have considerable influence on swallowing. For example, disturbed motility in the oral phase of swallowing is characteristic for IPD ${ }^{6,24}$. According to Nilsson et al., the prolongation of the oral-pharyngeal transit time is likely to reflect dysfunction caused by rigidity, bradykinesia (slowness of movement), and hypokinesia ${ }^{25}$. The lack of adaptive mechanisms to create space in the oral cavity, as seen in IPD patients, was interpreted as a form of hypokinesia by Wintzen et al. ${ }^{7}$. Both bradykinesia and rigidity affect the motor function of the tongue directly and adversely ${ }^{26,27}$. Therefore, its motor dysfunction -- such as repetitive upward and backward movements of the anterior tongue (lingual pumping) and prolonged ramping of the tongue in an attempt to initiate a swallow, with the posterior tongue remaining elevated against the palate -- may interfere with the entrance of the bolus into the pharynx ${ }^{24,28}$. Videofluoroscopy (VFS) is able to visualize manifestations such as residue on the tongue or residue in the anterior and lateral sulci, repeated pumping tongue motion, uncontrolled bolus or premature loss of liquid, and piecemeal deglutition. Pharyngeal phase disturbances have been reported too: reduced and delayed pharyngeal constrictor contraction; slow and reduced laryngeal excursion; slow and impaired true vocal fold closure; reduced epiglottic range of movement; and reduced and delayed opening of the upper esophageal sphincter $(\text { UES) })^{6,16,29,30}$.

It is preferable to conduct diagnostic examinations and logopedic dysphagia treatment during the "on" motor phase, which starts roughly 90 to 120 minutes after the intake of antiparkinsonian medication ${ }^{31}$, while L-dopa levels are high in the brain. Long-term use of L-dopa preparations may provoke motor complications, notably the involuntary movements called dyskinesia, and cause fluctuations in the response to medication. Parkinson patients may experience phase fluctuations ranging from a good response to medication and few symptoms ("on" motor phase) to no response and significant symptoms ("off" motor phase). 


\section{Treatment of oropharyngeal dysphagia in IPD}

Few reports have been published on the effect of therapies for oropharyngeal dysphagia in IPD. Physicians usually start with an antiparkinsonian medication like L-dopa. According to several studies, as noted above, oropharyngeal dysphagia responds to dopaminergic treatment only in a small proportion of patients, whereas other studies showed improved swallowing function after the intake of L-dopa ${ }^{19,32,33,34,35}$. In case of persistent dysphagic symptoms despite pharmacological treatment, alternative approaches such as logopedic or surgical treatment can be considered ${ }^{36}$. Several - including bolus modification, cueing (e.g., visual, auditory, tactile), exercise such as Lee Silverman Voice Treatment (LSVT) or expiratory muscle strength training (EMST), and video-assisted swallowing therapy (VAST) -- have been successful $28,37,38,39,40,41$. According to some studies, subjects with advanced IPD who underwent deep brain stimulation (DBS) have made significant improvement in swallowing ability ${ }^{42}$. New treatment techniques are being studied in patients with IPD: for instance, direct manipulation of cortical brain activity by repetitive transcranial magnetic stimulation (rTMS) has been serving as an efficacious complimentary rehabilitatory treatment for speech, language, and swallowing disorders ${ }^{43}$. These techniques warrant further investigation and optimalization.

Quality of life in IPD could be increased by the effective management of drooling, which is likely to come from an impaired swallowing function ${ }^{44}$. Patients with IPD often suffer from several other physical and psychological dysfunctions, prompting many visits to medical specialists or allied health professionals. In that light, a dysphagia rehabilitation program should take the other IPD-related treatments into account. In fact, multidisciplinary teams have been established in the outpatient clinic for oropharyngeal dysphagia over the past decade $^{45}$. This initiative has reduced the risk of complications from untreated oropharyngeal dysphagia -- such as aspiration pneumonia, which requires hospitalization and intravenous antibiotic treatment -- in a vulnerable patient population. The role of the team is emphasized in the literature, and screening tools and guidelines are being developed to help the professionals provide dysphagic patients with the best swallowing care available ${ }^{46}$. However, the protocols used in various clinical and instrumental examinations for swallowing disorders still need to be refined and standardized. Even if well-defined diagnostic protocols could be established, these would then have to be applied universally in daily clinical practice.

\section{Observer reliability for measurements in swallowing examinations}

A sufficient degree of observer reliability (intra- and interobserver) for measurements in swallowing examinations is crucial to clinical evaluation and research. However, there are few reports on intra- and interobserver reliability in VFS or for fiberoptic endoscopic 
evaluation of swallowing (FEES) measures derived from frame-by-frame analysis by expert raters. The observer reliability of measurements used to evaluate swallowing function in VFS or during FEES (each the 'gold standard' in its realm) remains a concern for future studies. Without good intra- and interobserver reliability, the measurements in VFS or FEES would be untrustworthy. In each new swallowing study, the measurements of temporal, spatial, or ordinal visuoperceptual variables must be deemed sufficiently reliable for subsequent statistical analysis. So, protocols for repeated measurements to evaluate the observer reliability should be described or referenced in the methodology section of each new study. Observer reliability might be insufficient when the raters are not given prior training in the definitions of temporal, spatial, or ordinal visuoperceptual variables ${ }^{47}$. Providing well-defined criteria for each level of a visuoperceptual VFS or FEES variable, in contrast, may lead to overall improvement in observer reliability ${ }^{15,48,49}$. Worldwide, numerous published papers on oropharyngeal dysphagia do not mention intra- and interobserver reliability. In many instances, the measurements in VFS or FEES had been conducted by a single rater. Unfortunately, the great effort put into these studies may be less valuable because of these methodological issues.

This thesis used FEES, among other evaluation techniques, as an instrumental assessment tool to evaluate the therapy effects of surface electrical stimulation of the neck in dysphagic IPD patients. Various standardized FEES protocols exist side by side. Yet there is no consensus on which one is the most preferable. Nor has it been agreed that a protocol should include the number of swallows and applied bolus consistencies or volumes to predict aspiration risk. The final prospective study included in this thesis was designed to estimate the probability of aspiration as a function of the number of swallow trials. It used a standardized FEES protocol of ten consecutive swallow trials of $10 \mathrm{cc}$ each for two different consistencies (thin and thick liquid). Those trials were conducted in a group of oncological and neurological patients suffering from oropharyngeal dysphagia.

\section{Objectives and outline of this thesis}

As reported in the systematic review in chapter 2, there is little evidence that any of the treatments for oropharyngeal dysphagia in IPD are effective. In that light, this thesis set out to evaluate the effects of a new treatment: surface electrical stimulation of the neck. An objective of this thesis was to determine the observer reliability (intra- and interobserver) of the outcome variables of the 'gold standard' in diagnostic tools, namely VFS and FEES, which are used to evaluate swallowing in IPD. Prior to the study of therapy effects in a quasi-randomized controlled trail, the pathophysiology of swallowing in IPD was studied. This was done by comparing IPD patients with ten age- and gender-matched healthy control subjects using VFS, as described in chapter 3 . In the experiment described in chapter 4, a single session of motor-level surface electrical stimulation of the neck used different electrode positions and electrical current conditions (current on/off) during VFS. The aim was to evaluate the effects of electrode position on the swallowing physiology in 
IPD patients. An elaborated experiment in a quasi-randomized controlled trial with 109 IPD patients is described in chapter 5. This trial evaluated the effect of different stimulation thresholds of surface electrical stimulation of the neck as adjunct to traditional logopedic dysphagia treatment in dysphagic IPD patients. The main instrumental assessment tools that were used to evaluate therapy effects are VFS and FEES.

Chapter 6 reports a prospective study estimating the probability of aspiration as a function of the number of swallow trials using a standardized FEES protocol of ten consecutive trials of $10 \mathrm{cc}$ each for two different consistencies (thin and thick liquid) in a group of oncological and neurological patients suffering from oropharyngeal dysphagia. Chapter 7 summarizes the main findings of this thesis and wraps it up with a discussion, conclusion, and some perspectives on the future. 


\section{References}

1. Parkinson J. An essay on the shaking palsy. London: Whittingham and Rowland; 1817.

2. de Rijk MC, Launer LJ, Berger K, Breteler MM, Dartigues JF, Baldereschi M, Fratiglioni L, Lobo A, MartinezLage J, Trenkwalder C, Hofman A. Prevalence of Parkinson's disease in Europe: a collaborative study of population-based cohorts-Neurologic Diseases in the Elderly Research Group. Neurology. 2000;54: S21-S23.

3. Jankovic J, Tolosa E. Parkinson's disease \& movement disorders, fifth edition. Philadelphia: Lippincott Williams \& Wilkins, Wolters Kluwer Business; 2007.

4. Hoehn NM, Yahr MD. Parkinsonism: onset, progression and mortality. Neurology. 1967;17:427-442.

5. Ertekin C, Tarlaci S, Aydogdu I, Kiylioglu N, Yuceyar N, Turman AB, Secil Y, Esmeli F. Electrophysiological evaluation of pharyngeal phase of swallowing in patients with Parkinson's disease. Movement disorders. 2002;17:942-949.

6. Nagaya M, Teruhiko K, Yamada T, Igata A. Videofluorographic study of swallowing in Parkinson's disease. Dysphagia. 1998;13:95-100.

7. Wintzen AR, Badrising UA, Roos RA, Vielvoye J, Liauw L. Influence of bolus volume on hyoid movements in normal individuals and patients with Parkinson's disease. Can J Neurol Sci. 1994;21:57-59.

8. Heijnen BJ, R Speyer, Baijens LWJ, Bogaardt HCA. Neuromuscular electrical stimulation versus traditional therapy in patients with Parkinson's disease and oropharyngeal dysphagia: Effects on quality of life. Dysphagia. 2012;27:336-345.

9. Leow LP, Huckabee ML, Anderson T, Beckert L. The impact of dysphagia on quality of life in ageing and Parkinson's disease as measured by the swallowing quality of life (SWAL-QOL) questionnaire. Dysphagia. 2010;25:216-220.

10. Westergren A, Unosson M, Ohlsson O, Lorefält B, Hallberg IR. Eating difficulties, assisted eating and nutritional status in elderly ( $>$ or $=65$ years) patients in hospital rehabilitation. Int J Nurs Stud. 2002;39:341-351.

11. Markus HS, Tomkins AM, Stern GM. Increased prevalence of undernutrition in Parkinson's disease and its relationship to clinical disease parameters. J Neural Transm Park Dis Dement Sect. 1993;5:117-125.

12. Lieu PK, Chong MS, Seshadri R. The impact of swallowing disorders in the elderly. Ann Acad Med Singapore. 2001;30:148-154.

13. Steele CM, Greenwood C, Ens I, Robertson C, Seidman-Carlson R. Mealtime difficulties in a home for the aged: not just dysphagia. Dysphagia. 1997;12:43-50.

14. Veldee MS, Peth LD. Can protein-calorie malnutrition cause dysphagia? Dysphagia. 1992;7:86-101.

15. Baijens L, Speyer R, Lima Passos V, Pilz W, Roodenburg N, Clavé P. Swallowing in Parkinson patients versus healthy controls: Reliability of measurements in videofluoroscopy. Gastroenterol Res Pract. 2011;2011: 380682.

16. Ali GN, Wallace KL, Schwartz R, Decarle DJ, Zagami AS, Cook IJ. Mechanisms of oral-pharyngeal dysphagia in patients with Parkinson's disease. Gastroenterology. 1996;110:383-392.

17. Fernandez HH, Lapane KL. Predictors of mortality among nursing home residents with a diagnosis of Parkinson's disease. Med Sci Monit. 2002;8:CR241-CR246.

18. Gorell JM, Johnson CC, Rybicki BA. Parkinson's disease and its comorbid disorders: an analysis of Michigan mortality data, 1970 to 1990. Neurology. 1994;44:1865-1868.

19. Hunter PC, Crameri J, Austin S, Woodward MC, Hughes J. Response of parkinsonian swallowing dysfunction to dopaminergic stimulation. J Neurol Neurosurg Psychiatry. 1997;63:579-583.

20. Melo A, Monteiro L. Swallowing improvement after levodopa treatment in idiopathic Parkinson's disease: Lack of evidence. Parkinsonism Relat Disord. 2013;19:279-281.

21. Menezes C, Melo A. Does levodopa improve swallowing dysfunction in Parkinson's disease patients? J Clin Pharm Ther. 2009;34:673-676.

22. Braak H, Ghebremedhin E, Rub U, Bratzke H, Del Tredici K. Stages in the development of Parkinson's disease-related pathology. Cell Tissue Res. 2004;318:121-134.

23. Suntrup S, Teismann I, Bejer J, Suttrup I, Winkels M, Mehler D, Pantev C, Dziewas R, Warnecke T. Evidence for adaptive cortical changes in swallowing in Parkinson's disease. Brain. 2013;136:726-738.

24. Bird MR, Woodward MC, Gibson EM, Phyland DJ, Fonda D. Asymptomatic swallowing disorders in elderly patients with Parkinson's disease: A description of findings on clinical examination and videofluoroscopy in sixteen patients. Age Ageing. 1994;23:251-254. 
25. Nilsson H, Ekberg O, Olsson R, Hindfelt B. Quantitative assessment of oral and pharyngeal function in Parkinson's disease. Dysphagia. 1996;11:144-150.

26. Bushmann M, Dobmeyer SM, Leeker L, Perlmutter JS. Swallowing abnormalities and their response to treatment in Parkinson's disease. Neurology. 1989;39:1309-1314.

27. Edwards LL, Quigley EM, Pfeiffer RF. Gastrointestinal dysfunction in Parkinson's disease: frequency and pathophysiology. Neurology. 1992;42:726-732.

28. Logemann JA, Gensler G, Robbins JA, Lindblad AS, Brandt D, Hind JA, Kosek S, Dikeman K, Kazandjian M, Gramigna GD, Lundy D, McGarvey-Toler S, Miller Gardner PJ. A randomized study of three interventions for aspiration of thin liquids in patients with dementia or Parkinson's disease. J Speech Lang Hear Res. 2008;51:173-183.

29. Leopold NA, Kagel MC. Prepharyngeal dysphagia in Parkinson's disease. Dysphagia. 1996;11:14-22.

30. Leopold NA, Kagel MC. Laryngeal deglutition movement in Parkinson's disease. Neurology. 1997;48: 373-376.

31. Wajsbort J. The "off-on" phenomenon during treatment of Parkinson's disease with levodopa. J Neurol. 1977;15:59-66.

32. Calne DB, Shaw DG, Spiers AS, Stern GM. Swallowing in Parkinsonism. Br. J. Radiol. 1970;43:456-457.

33. Fuh JL, Lee RC, Wang SJ, Lin CH, Wang PN, Chiang JH, Liu HC. Swallowing difficulty in Parkinson's disease. Clin Neurol Neurosurg. 1997;99:106-112.

34. Sutton JP. Dysphagia in Parkinson's disease is responsive to levodopa. Parkinsonism Relat Disord. 2013; 19:282-284.

35. Tawadros PB, Cordato D, Cathers I, Burne JA. An electromyographic study of parkinsonian swallowing and its response to levodopa. Mov Disord. 2012;27:1811-1815.

36. Nagaya M, Kachi T, Yamada T. Effect of swallowing training on swallowing disorders in Parkinson's disease. Scand J Rehab Med. 2000;32:11-15.

37. Pinnington LL, Muhiddin KA, Ellis RE, Playford ED: Non-invasive assessment of swallowing and respiration in Parkinson's disease. J Neurol. 2000;247:773-777.

38. El Sharkawi A, Ramig L, Logemann JA, Pauloski BR, Rademaker AW, Smith CH, Pawlas A, Baum S, Werner C. Swallowing and voice effects of Lee Silverman Voice Treatment (LSVT): a pilot study. J Neurol Neurosurg Psychiatry. 2002;72:31-36.

39. Troche MS, Okun MS, Rosenbek JC, Musson N, Fernandez HH, Rodriguez R, Romrell J, Pitts T, WheelerHegland KM, Sapienza CM. Aspiration and swallowing in Parkinson disease and rehabilitation with EMST: a randomized trial. Neurology. 2010;75:1912-1919.

40. Pitts T, Bolser D, Rosenbek J, Troche M, Okun MS, Sapienza C. Impact of expiratory muscle strength training on voluntary cough and swallow function in Parkinson disease. Chest. 2009;135:1301-1308.

41. Manor Y, Mootanah R, Freud D, Giladi N, Cohen JT. Video-assisted swallowing therapy for patients with Parkinson's disease. Parkinsonism Relat Disord. 2013;19:207-211.

42. Silbergleit AK, LeWitt P, Junn F, Schultz LR, Collins D, Beardsley T, Hubert M, Trosch R, Schwalb JM. Comparison of dysphagia before and after deep brain stimulation in Parkinson's disease. Mov Disord. 2012;27:1763-1768.

43. Barwood $\mathrm{CH}$, Murdoch BE. rTMS as a treatment for neurogenic communication and swallowing disorders. Acta Neurol Scand. 2013;127:77-91.

44. Kalf JG, Smit AM, Bloem BR, Zwarts MJ, Munneke M. Impact of drooling in Parkinson's disease. J Neurol. 2007;1227-1232.

45. Baijens LWJ. Multidisciplinaire polikliniek voor dysfagie. Nederlands Tijdschrift voor Keel-NeusOorheelkunde. $18^{\mathrm{e}}$ jaargang nummer 1 januari 2012 p5-6.

46. Kaspar K, Ekberg O. Identifying vulnerable patients: role of the EAT-10 and the multidisciplinary team for early intervention and comprehensive dysphagia care. Nestle Nutr Inst Workshop Ser. 2012;72:19-31.

47. McCullough GH, Wertz RT, Rosenbek JC, Mills RH, Webb WG, Ross KB. Inter- and intrajudge reliability for videofluoroscopic swallowing evaluation measures. Dysphagia. 2001;16:110-118.

48. Tohara h, Nakane A, Murata S, Mikushi S, Ouchi Y, Wakasugi Y, Takashima M, Chiba Y, Uematsu H. Interand intra-rater reliability in fiberoptic endoscopic evaluation of swallowing. Journal of Oral Rehabilitation. 2010;37:884-891.

49. Baijens L, Barikroo A, Pilz Walmari. Intrarater and interrater reliability for measurements in videofluoroscopy of swallowing. Eur J Radiol. 2013;82:1683-1695. 
Chapter 2

Effects of therapy for dysphagia in Parkinson's disease: systematic review

Laura W.J. Baijens

Renée Speyer

Dysphagia 2009;24:91-102
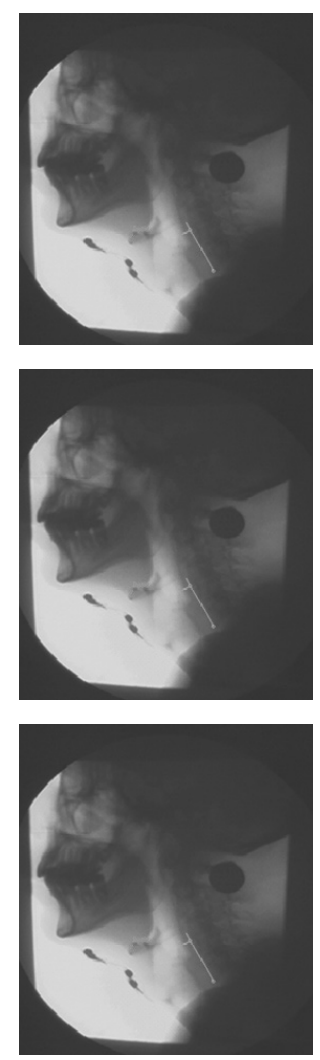


\section{Abstract}

This systematic review explores the effects of dysphagia treatment for Parkinson's disease. The review includes rehabilitative, surgical, pharmacologic, and other treatments. Only oropharyngeal dysphagia is selected for this literature search, excluding dysphagia due to esophageal or gastric disorders. The effects of deep brain stimulation on dysphagia are not included. In general, the literature concerning dysphagia treatment in Parkinson's disease is rather limited. Most effect studies show diverse methodologic problems. Multiple case studies and trials are identified by searching biomedical literature databases PubMed and Embase, and by hand-searching reference lists. The conclusions of most studies cannot be compared with one another because of heterogeneous therapy methods and outcome measures. Further research based on randomized controlled trials to determine the effectiveness of different therapies for dysphagia in Parkinson's disease is required. 


\section{Introduction}

Dysphagia is a frequent symptom of Parkinson's disease ${ }^{1-3}$ and is often associated with considerable morbidity and mortality due to nutritional and pulmonary complications ${ }^{1,2}$. Dysphagia is observed more often in patients with an advanced stage of Parkinson's disease $^{4}$. In the literature, the prevalence of dysphagia varies from 18.5 to $100 \%{ }^{2,3}$. However, as many as $15 \%$ of the patients with Parkinson's disease show silent aspiration and do not complain of dysphagia ${ }^{1}$. The pathologic mechanisms of oropharyngeal dysphagia in Parkinson's disease are not quite clear. Oropharyngeal bradykinesia and rigidity, incomplete cricopharyngeal relaxation, reduced cricopharyngeal opening, and delayed initiation of the swallowing reflex have been suggested as possible mechanisms of dysphagia in this patient population ${ }^{1,4}$. Among the different therapies for dysphagia in Parkinson's disease, rehabilitative treatment (swallowing training), cricopharyngeal sphincterotomy, and pharmacologic therapies have been reported.

The number of reports on the effects of therapies for dysphagia in Parkinson's disease is still rather small. This article is a systematic review of the literature on the effects of treatment for dysphagia in Parkinson's disease. Dean et al. ${ }^{5}$ published a systematic review on nonpharmacologic therapies for dysphagia in Parkinson's disease; however, the present systematic review incorporates all therapies available for oropharyngeal dysphagia in Parkinson's disease, including an update on rehabilitative therapies. The different types of therapy are grouped into four main therapy groups: rehabilitative, surgical, pharmacologic, and other.

\section{Methods}

The two authors independently carried out the literature search using the electronic literature databases Embase and PubMed. Differences in their search were settled by discussion. The search was limited to English, German, French, Spanish, and Dutch language publications. In Embase the Mesh terms swallowing or dysphagia were combined with Parkinsonism or Parkinson-disease. In PubMed the Mesh terms Parkinson disease or Parkinson disease, secondary or Parkinsonian disorders were combined with deglutition disorders. To identify the most recent publications, the search was supplemented by using free-text words (truncation or wildcard) in Embase and PubMed (for the period after January 2005 up to May 2008): deglut* or swallow* or dysphag* were combined with hypokinetic syndrome or Parkinson* or Paralysis agitans. A total of 602 articles were found in Embase and 258 articles in PubMed. Only articles on the effects of therapy for oropharyngeal dysphagia in Parkinson's disease were included. Studies describing heterogeneous subject populations that included patients with Parkinson's disease and patients with other neurologic diseases were excluded ${ }^{6}$, except those studies that presented both pre- and posttreatment data per subgroup ${ }^{7}$. Patients receiving deep brain stimulation were excluded. It was decided to exclude deep brain stimulation 
because dysphagia has been described as a side effect of this therapy ${ }^{8}$. Studies using a subject population of fewer than three were excluded ${ }^{9-11}$. The relevance and level of evidence of studies based on populations of fewer than three subjects is doubtful. Percutaneous endoscopic gastrostomy (PEG) tube feeding was excluded as treatment for dysphagia because it is a feeding substitute. There were no limitations on disease duration or disease severity. Articles with subjects of all ages and any duration of treatment were selected. After a first selection based on abstracts, a definitive inclusion was made using the original articles. The reference lists of all the included articles were searched for additional literature. This search did not provide addition studies. In conclusion, 16 articles were included in this review.

\section{Results}

Table 2.1 presents a summary of the 16 articles that describe rehabilitative, surgical, pharmacologic, or other therapies for dysphagia in Parkinson's disease. The first column of the table represents the level of evidence. To rate the study quality, the $A B C$ rating scale according to Siwek et al. ${ }^{12}$ was used. Level A refers to high-quality randomized controlled trials; level $B$ refers to well-designed, nonrandomized clinical trials, and level $C$ refers to consensus or expert opinions. Categories A and B were subdivided into two groups according to the way data were handled. The first group used statistical analyses for comparing pre- versus posttherapy data. The second group used descriptive statistics to evaluate the therapy outcome. Level $\mathrm{C}$ reflects an expert's opinion without presentation of any statistical data. Authors were listed in alphabetical order. The following data were summarized (if present) for each article: the number of patients, the disease severity based on the Hoehn and Yahr scale $(\mathrm{H}-\mathrm{Y} \text { scale })^{13}$, the kind of therapy used, the evaluation techniques, outcome parameters, blinded rater(s), "on/off" motor phase, and the authors' key findings. The fluctuation in daily performance of Parkinson patients on long-term Ldopa therapy is known as the "on/off" phenomenon ${ }^{14}$. The number of subjects refers to the group of subjects on which the study results were based, i.e., dropouts were excluded. All studies were described briefly.

\section{Rehabilitative treatment (swallowing training)}

Studies to determine the effects of swallowing training in Parkinson's disease are extremely rare. El Sharkawi et al. ${ }^{15}$ studied the effects of 1 month of Lee Silverman Voice Treatment (LSVT) on swallowing and voice in eight patients with idiopathic Parkinson's disease and dysphagia. The treatment was conducted four times a week at 50-60 minutes per session. The effectiveness of the LSVT was evaluated by comparing pre- and posttherapy measurements, including a lateral videofluoroscopy of swallowing (VFS) conducted at the same time of day and medicine cycle for each patient. One rater, who was blinded to the therapy used, performed the swallow analysis. 


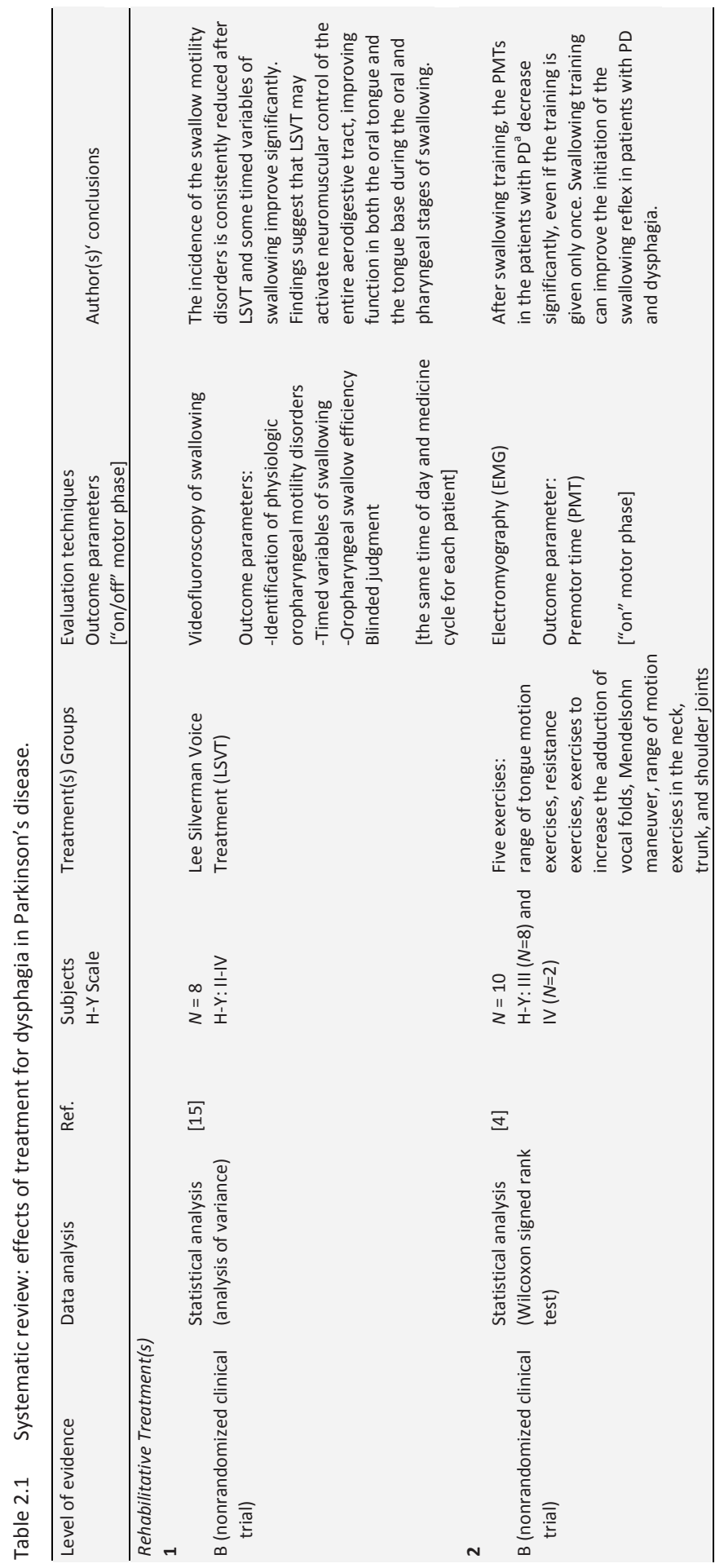




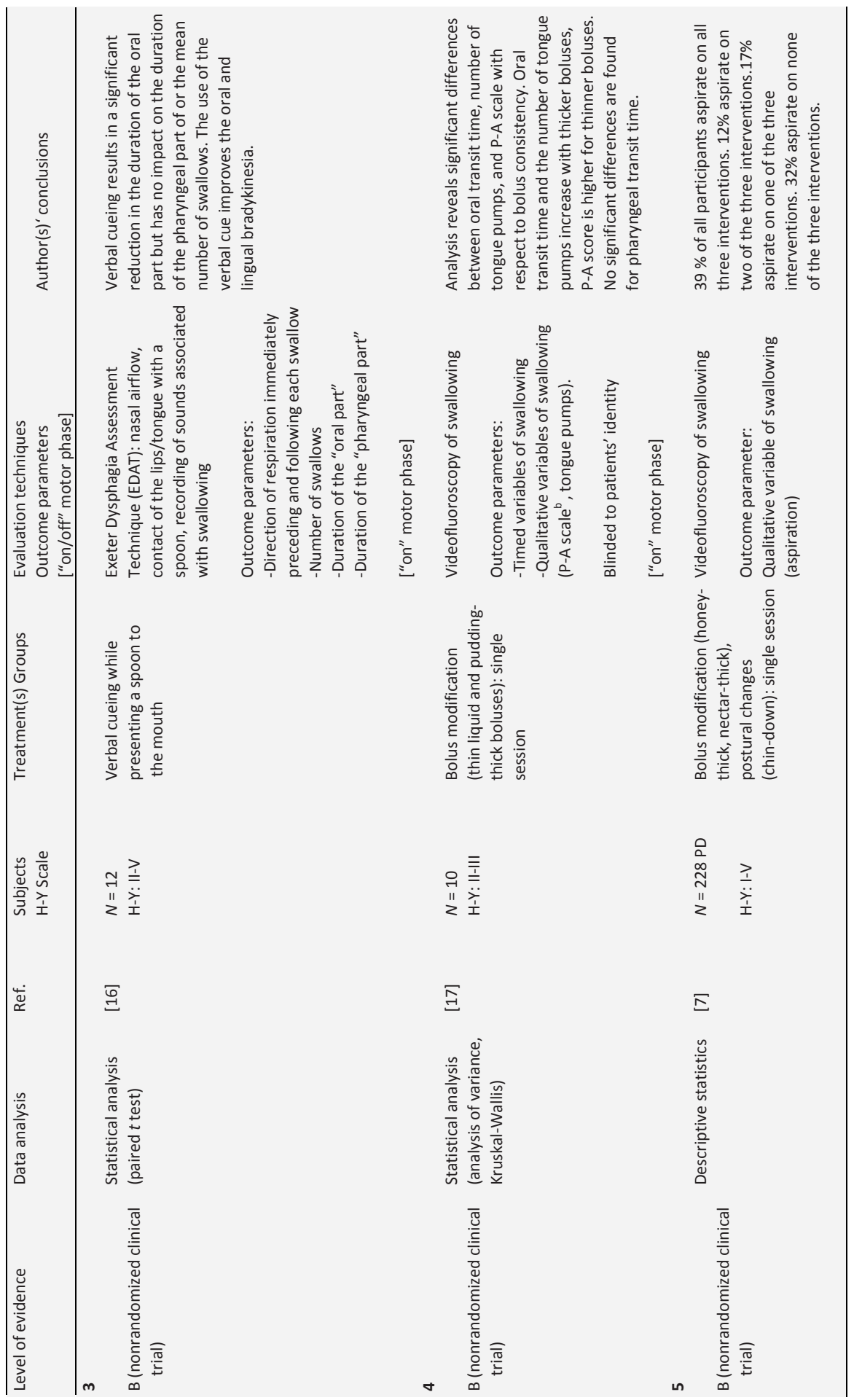




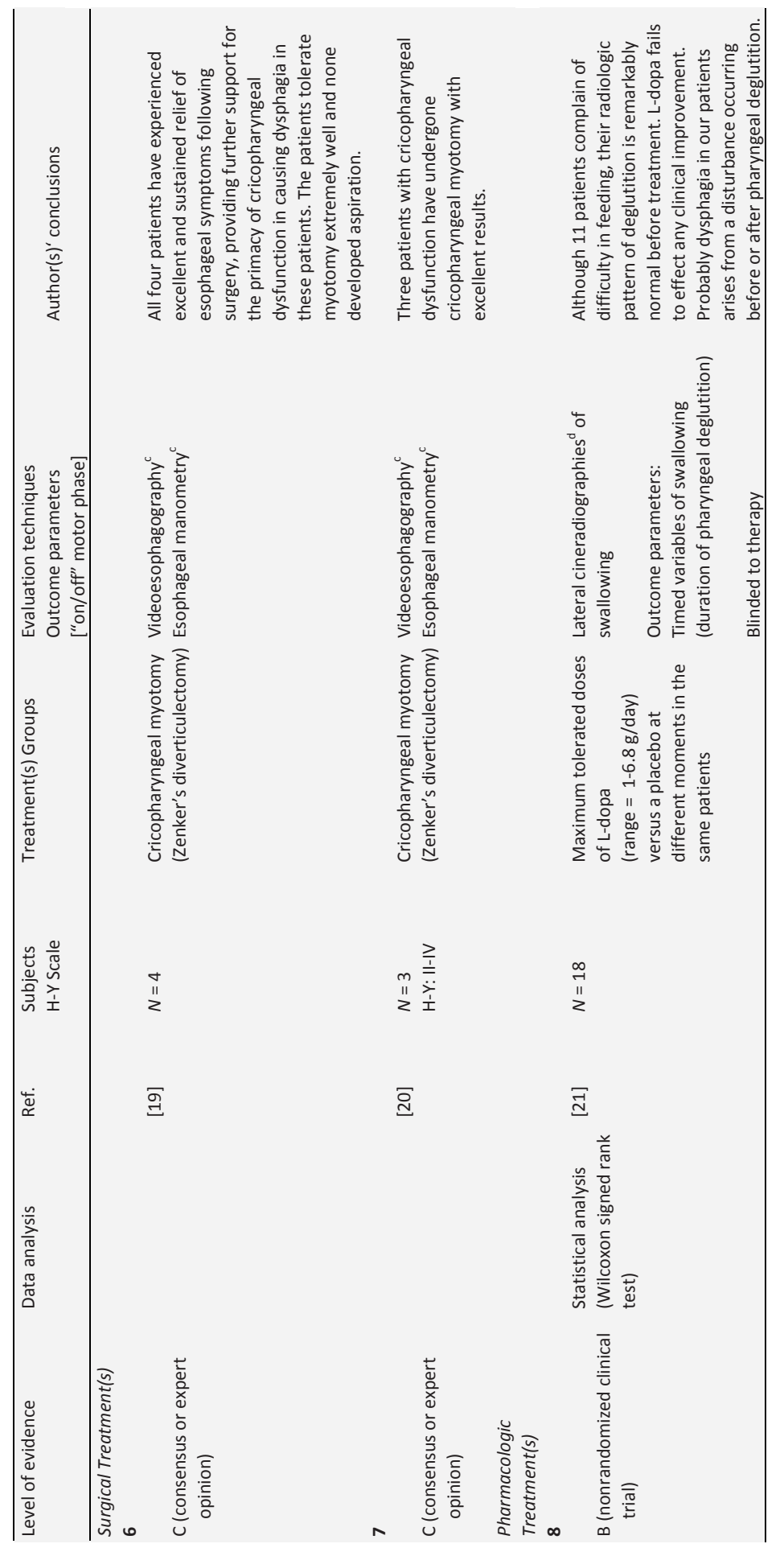




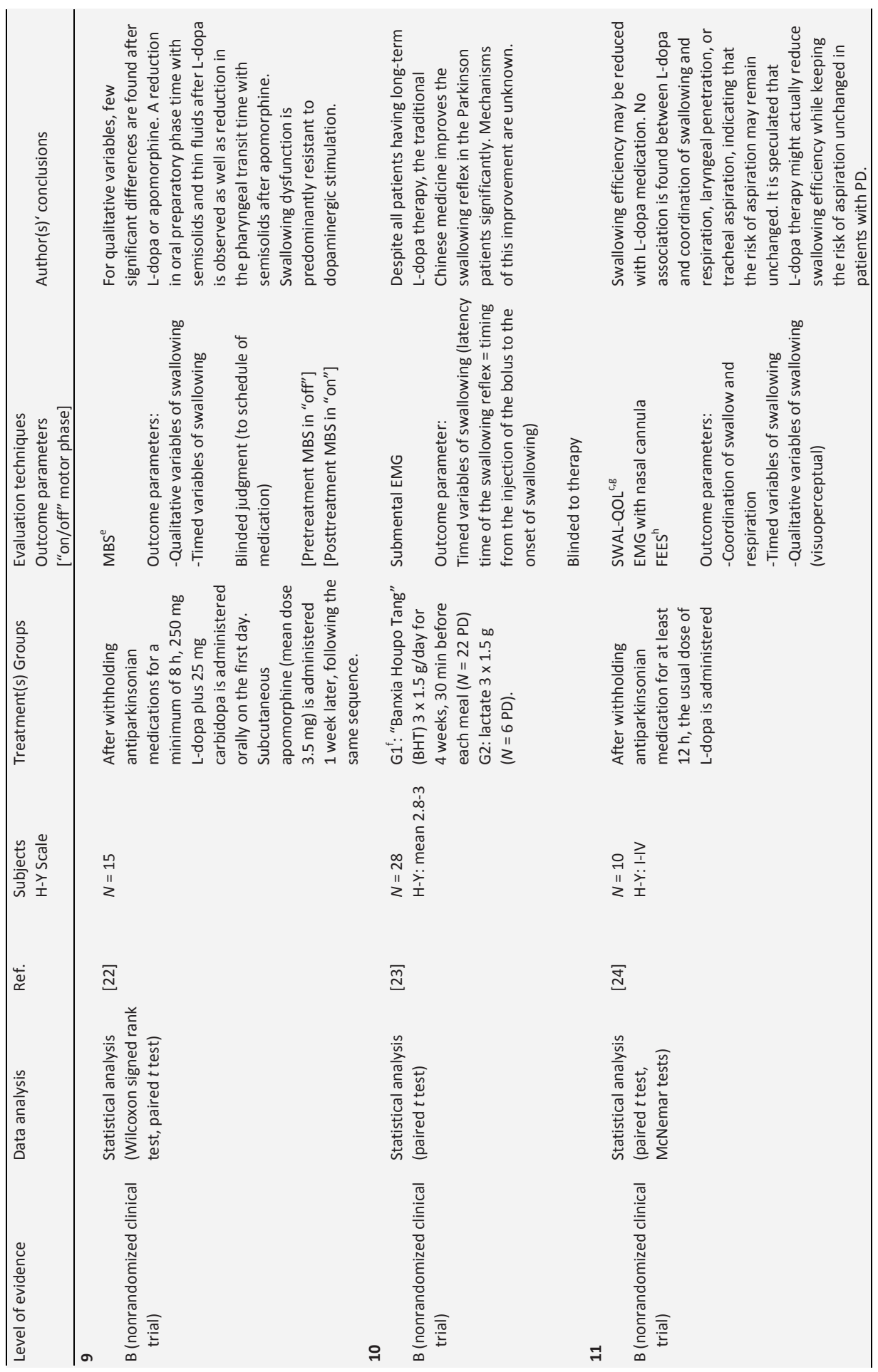




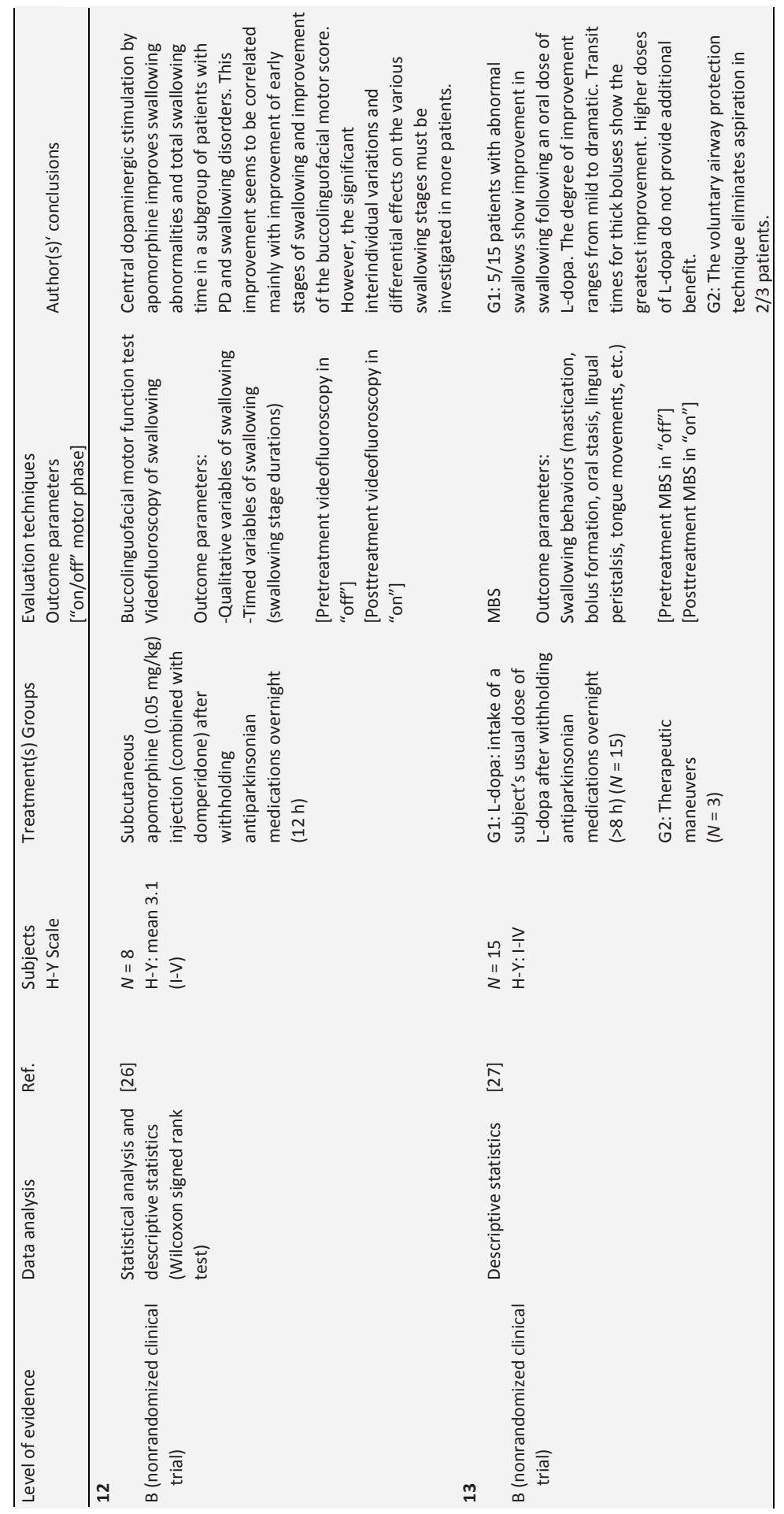




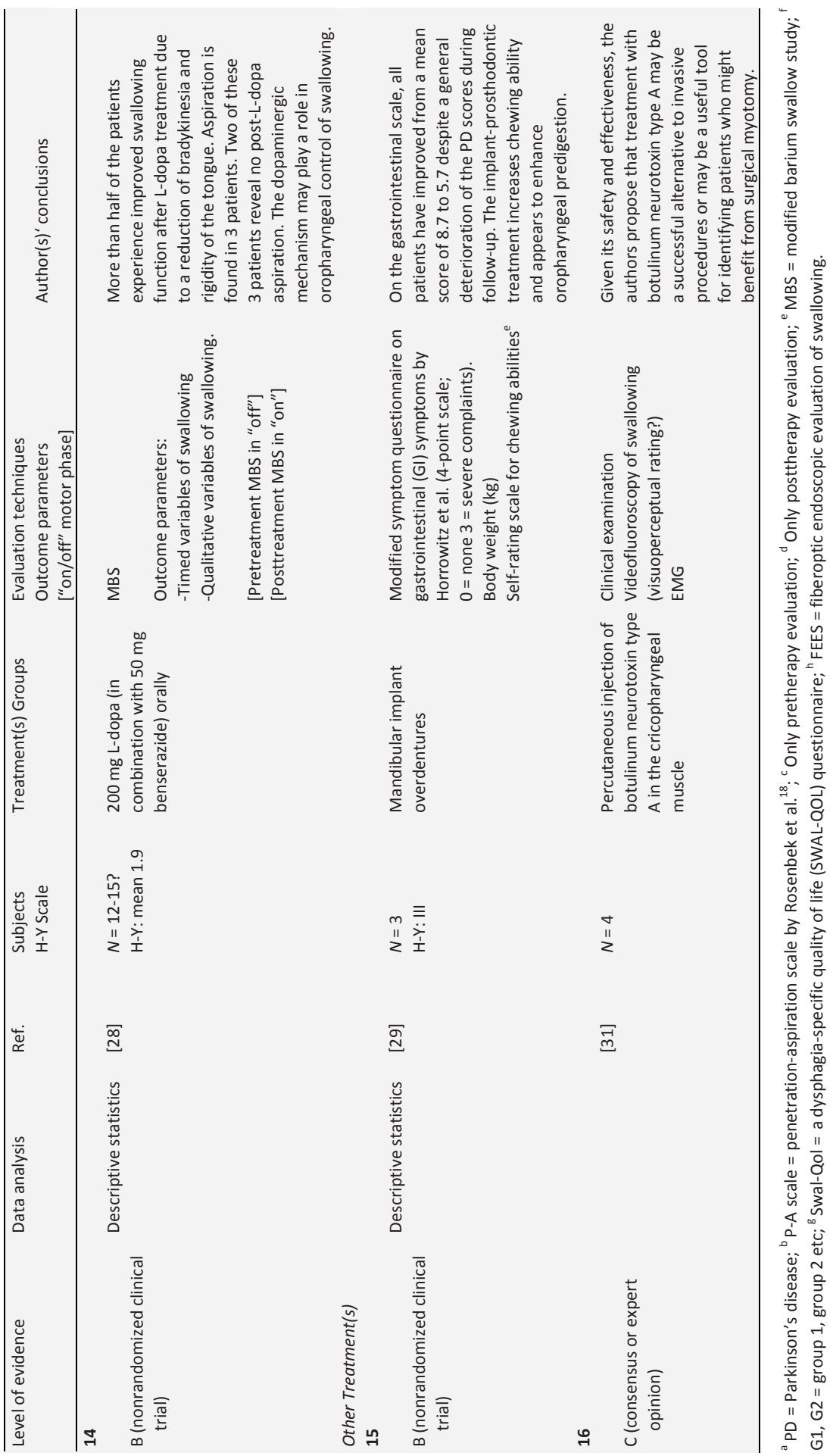


Outcome parameters consisted of temporal and qualitative measures of swallowing. Disease severity according to the $\mathrm{H}-\mathrm{Y}$ scale and statistical analysis were described. After 1 month of LSVT, the authors concluded that LSVT seemed to be effective in improving neuromuscular control of the entire upper aerodigestive tract, improving oral tongue and tongue base function during the oral and pharyngeal phases of swallowing.

In a study by Nagaya et al. ${ }^{4}$, the authors tried to determine whether swallowing training, consisting of five motor function/behavioral exercises, improved swallowing function in ten patients with Parkinson's disease and dysphagia. The swallowing training took about 20 minutes. There was only one session of exercises. The premotor time (PMT) was evaluated pre- and posttherapy during the "on" motor phase using electromyography (EMG). The PMT was defined as the latency of EMG activity when a specific movement was requested in response to an imperative stimulus, reflecting the time for information processing in the sensorimotor pathways. The modified barium swallow study (MBS) was performed only before treatment to assess the oral and pharyngeal functions in patients with Parkinson's disease. All examinations were performed during the "on" motor phase. Disease severity was mentioned ( $\mathrm{H}-\mathrm{Y}$ scale) and a control population of 12 healthy volunteers was used. It was not mentioned whether the pre- versus posttreatment EMG results were scored blinded to the schedule of treatment. A statistically significant decrease in PMT in patients with Parkinson's disease was observed after swallowing training, even though the training was done only once. The initiation of the swallowing reflex in patients with Parkinson's disease and dysphagia could be improved by swallowing training.

In the report by Pinnington et al. ${ }^{16}$, the effects of verbal cueing on the swallowing function while presenting a spoon of liquid to the mouth ("on" motor phase) were evaluated by means of the Exeter Dysphagia Assessment Technique (EDAT) in 12 patients with Parkinson's disease compared to 14 control patients on the orthogeriatric ward. The EDAT recorded nasal airflow, contact of the lips or tongue with a spoon, and sounds associated with swallowing. Disease severity was scored according to the $\mathrm{H}-\mathrm{Y}$ disability scale and the Unified Parkinson's Disease Rating Scale. It was not mentioned whether the rating during the EDAT was performed blinded to diagnosis and therapy. After statistical analysis the authors concluded that the use of verbal cueing resulted in a significant reduction in the duration of the oral part of swallowing, but had no impact on the duration of the pharyngeal part of swallowing or the mean number of swallows.

Troche et al. $^{17}$ evaluated the effect of bolus consistency on timing and safety of swallowing in patients with Parkinson's disease. The severity of the disease was scored using the H-Y scale. During VFS, ten Parkinson patients completed a dry swallow, six $5 \mathrm{cc}$ trials of pudding-thick liquid, and six $5 \mathrm{cc}$ trials of thin liquid. The trials were presented in random order. Swallowing was analyzed using qualitative (penetration-aspiration scale [P-A] by Rosenbek et al. ${ }^{18}$ and number of tongue pumps) and quantitative (timed variables) swallowing parameters. Patients were tested 1 hour after medication during the "on" motor phase. The examiner was blinded to the patients' identity. Analysis revealed significant differences between oral transit time, number of tongue pumps, and P-A scale 
with respect to bolus consistency. Oral transit time and the number of tongue pumps increased with thicker boluses and the P-A score was higher for thinner boluses. No significant differences were found for pharyngeal transit time.

Logemann et al. ${ }^{7}$ performed a study to identify which of three treatments for aspiration on thin liquids (chin-down posture, nectar-thickened liquids, or honey-thickened liquids) resulted in the most successful immediate elimination of aspiration during a VFS study in patients with dementia and/or Parkinson's disease. Two hundred twenty-eight Parkinson patients underwent all three interventions in randomly assigned order. The H-Y scale was used. The outcome parameter during VFS was a qualitative variable (aspiration) of swallowing. No information about the "on/off" motor phase during measurements was given. Thirty-nine percent of participants with Parkinson's disease without dementia aspirated on all three interventions. Twelve percent aspirated on two of the three interventions. Seventeen percent aspirated on one of the three interventions. Thirty-two percent aspirated on none of the three interventions. Based on these descriptive results the authors concluded that the most frequently successful intervention to eliminate thinliquid aspiration immediately was the honey-thickened liquid, followed closely by the nectar-thickened liquid and then the chin-down posture.

\section{Surgical treatment}

Two articles provided anecdotal data about surgical treatment for dysphagia in Parkinson's disease. In the articles by Born et al. ${ }^{19}$ and Byrne et al. ${ }^{20}$, cricopharyngeal sphincterotomy was performed in very small groups of patients. Born et al. ${ }^{19}$ described four patients who experienced excellent and sustained relief of esophageal symptoms following surgery, providing further support for the primacy of cricopharyngeal dysfunction in causing dysphagia in these patients. The patients tolerated myotomy extremely well and none developed aspiration. Byrne et al. ${ }^{20}$ studied three patients with cricopharyngeal dysfunction who had undergone cricopharyngeal myotomy with excellent results. Only pretreatment measurements consisting of manometry and videoesophagography were performed in both studies. No posttreatment measurements were described, although the authors' own conclusions were included. In contrast to Born et al. ${ }^{19}$, Byrne et al. ${ }^{20}$ mentioned disease severity ( $\mathrm{H}-\mathrm{Y}$ scale).

\section{Pharmacologic treatment}

Seven articles provided information about the effects of antiparkinsonian drugs on the swallowing function.

Calne et al. ${ }^{21}$ studied the effect of maximum tolerated doses of L-dopa on swallowing (range $=1-6.8 \mathrm{~g} /$ day) compared to a placebo in 18 patients with idiopathic Parkinsonism by means of lateral cineradiography. Data were gathered for both L-dopa and placebo use in the same patients. The outcome parameter was duration of pharyngeal deglutition. Patients and the radiologist were blinded to therapy. Disease severity and "on/off" motor phase were not mentioned. Statistical analysis showed no significant difference between 
the pharyngeal transit times during the intake of L-dopa and of placebo. However, all patients showed remarkably normal radiologic patterns of deglutition before the onset of treatment; only 11 of 18 patients suffered from subjective dysphagia.

Hunter et al. ${ }^{22}$ studied the degree of dopaminergic response of swallowing dysfunction in 15 patients with Parkinson's disease. After a minimum of 8 hours of withdrawal from antiparkinsonian medication, the patients received a single oral dose of $250 \mathrm{mg}$ of L-dopa plus $25 \mathrm{mg}$ of carbidopa on the first study day. The pretreatment MBS was performed during the "off" motor phase and the posttreatment MBS during the "on" motor phase. One week later the sequence of medication was repeated using subcutaneous apomorphine (mean dose=3.5 mg). Outcome parameters consisted of qualitative measures of swallowing function and timed components of swallowing. Two blinded judges performed the rating of the MBS. No information was given about the initial severity of the disease of the included patients. For qualitative variables, few significant differences were found after L-dopa or apomorphine. A significant reduction in oral preparatory phase time with semisolids and thin fluids after L-dopa was observed, as well as a reduced pharyngeal transit time with semisolids after apomorphine. Using statistical analysis, the authors concluded that swallowing dysfunction was predominantly resistant to dopaminergic stimulation.

The study by Iwasaki et al. ${ }^{23}$ described the positive therapy effects of traditional Chinese medicine "Banxia Houpo Tang" (BHT) in improving the swallowing reflex of patients with Parkinson's disease. They studied 28 patients with a defined $\mathrm{H}-\mathrm{Y}$ score and compared them to five healthy controls of comparative age. They divided the patients with Parkinson's disease into two groups. Twenty-two patients and the healthy controls were given BHT $1.5 \mathrm{~g}$ three times a day for 4 weeks at 30 minutes before each meal and six patients were given the same amount of lactate. The evaluation tool used was submental EMG. Latency time of the swallowing reflex (timing from the injection of the bolus to the onset of swallowing) was used as the outcome parameter. The examiners did not know which patients took BHT or lactate. Whether patients were examined during an "on/off" motor phase was not mentioned. The EMG examinations before and after treatment were performed at 11 a.m. After therapy a statistically significant decrease of swallowing reflex time was demonstrated in the patient group receiving BHT. It remains unclear which components in the extract of dried plants were responsible for the improvement of the swallowing function.

Lim et al. ${ }^{24}$ studied the effect of the usual dose of L-dopa on the coordination of swallowing and respiration in ten patients with Parkinson's disease. The patients were randomly assigned to group 1 or group 2 . Group 1 had the first assessment session during the "on" motor phase and the second assessment session during the "off" motor phase. Group 2 had the first session during the "off" motor phase and the second during the "on" motor phase. The two sessions during "on" and "off" were performed in the morning at least 1 week apart. The assessment consisted of EMG with a nasal cannula and an endoscopic evaluation of swallowing (FEES). A dysphagia quality-of-life questionnaire (SWAL-QOL) was filled out only before treatment ${ }^{25}$. Disease severity according to the $\mathrm{H}-\mathrm{Y}$ 
scale and statistical analysis were described. No information was given whether the results of the EMG and FEES were scored blinded to therapy. After statistical analysis the authors concluded that swallowing efficiency might be reduced with L-dopa medication. No association was found between L-dopa and coordination of swallowing and respiration, laryngeal penetration, or tracheal aspiration, indicating that the risk of aspiration may remain unchanged. According to the authors, further studies comprising larger numbers of patients are needed to determine whether the trend in swallowing efficiency is a true effect and to confirm if there really is no difference in risk of aspiration between "on" and "off" states of L-dopa.

Tison et al. ${ }^{26}$ studied the effects of apomorphine (in combination with domperidone) on buccolinguofacial motoricity and on various swallowing stages by using VFS in eight patients with Parkinson's disease and dysphagia. The severity of the disease was scored by the $\mathrm{H}-\mathrm{Y}$ scale. The pre- and posttherapy assessments included a buccolinguofacial motor function test and VFS analyzed using qualitative and quantitative swallowing parameters. Only a subjective self-rating of the dysphagia severity on a 10-point scale was carried out pretreatment. Pretreatment measurements were performed during the "off" motor phase after overnight withdrawal of medication. Posttreatment measurements were performed during the "on" motor phase. It was not mentioned whether rating of the VFS examinations was performed blinded to therapy or by how many judges. After statistical analysis the authors found statistically significant results indicating that central dopaminergic stimulation by apomorphine improves swallowing abnormalities and restricts total swallowing time in a subgroup of patients with Parkinson's disease and swallowing disorders. This improvement was correlated mainly with improvement of the early stages of swallowing and improvement of the buccolinguofacial motor score. However, the interindividual variations and differential effects on the various swallowing stages must be investigated in a larger patient population in order to detect statistically significant differences.

In contrast to the previous four articles that used statistical analysis, the following two studies provided descriptive data about the effects of L-dopa on the swallowing function. In the study by Bushmann et al. ${ }^{27}$, the effects of the intake of the usual dose of L-dopa were evaluated by means of pre- and posttreatment MBS in 20 patients with Parkinson's disease. The severity of the disease was scored by the H-Y scale. The number of subjects with Parkinson's disease who were included in the data analysis was not clear. Only 15 of 20 patients had abnormal swallows on MBS before therapy. A second therapy consisting of a voluntary airway protection technique for the mechanical modification of swallowing was applied to three patients. It was not clear whether these three patients also participated in the L-dopa trial. An oral motor screening exam and Logemann's bedside dysphagia evaluation were performed only pretherapy. A control group of 13 subjects with no clinical evidence of neurologic or swallowing abnormality was recruited. The pretreatment MBS was performed during the "off" motor phase and the posttreatment MBS during the "on" motor phase. A variety of swallowing behaviors were assessed during the MBS by two speech pathologists, of which one was blinded to diagnosis. A clear 
improvement in swallowing in 5 of the 15 patients following an oral dose of L-dopa was observed. The voluntary airway protection technique eliminated aspiration in two of three patients. According to the authors, higher doses of L-dopa did not provide additional benefit.

Fuh et al. ${ }^{28}$ examined the oropharyngeal swallowing ability in 19 Parkinson's disease patients using MBS before and after administering oral L-dopa (200 mg) in combination with benserazide $(50 \mathrm{mg})$. Salivation and subjective swallowing difficulty were graded on a 5-point scale pretreatment. Salivation was graded as follows: $0=$ normal, $1=$ slight but definite excessive saliva in mouth with possible nighttime drooling, 2=moderately excessive saliva with minimal drooling, 3=marked excessive saliva with some drooling, $4=$ marked drooling requiring constant use of tissue or handkerchief. Subjective swallowing difficulty was scored on a 5-point scale as well: 0=normal, 1=rare choking, $2=$ occasional choking, $3=$ requiring soft food, 4=requiring nasogastric or percutaneous endoscopic gastrostomy (PEG) tube feeding. The pretreatment MBS was performed during the "off" motor phase and the posttreatment MBS during the "on" motor phase. The severity of Parkinson's disease was determined using the $\mathrm{H}-\mathrm{Y}$ scale and the Schwab and England scores. The judge who rated the radiologic recordings was blinded to the severity of the disease but was not blinded to the time the drugs were taken. Twelve of 19 patients had abnormal swallows on MBS. Six of these 12 patients showed improvement after taking L-dopa. Three of 19 patients showed aspiration on MBS. Two of these three revealed no post-L-dopa aspiration. It was not clear whether these three patients overlapped with the 12 patients with abnormal swallows. Based on descriptive statistics, the authors concluded that more than half of the patients experienced improved swallowing function after L-dopa treatment due to a reduction of bradykinesia and rigidity of the tongue.

\section{Other treatments}

Heckmann et al. $^{29}$ studied the benefit of using dental implants combined with overdentures to improve chewing and preingestion capacity in three severely handicapped Parkinson patients. Disease severity was scored according to the H-Y scale. Gastrointestinal (GI) symptoms were assessed pre- and posttreatment using a modified symptom questionnaire described by Horrowitz et al. ${ }^{30}$. Five GI symptoms, i.e., abdominal bloating, heartburn, dysphagia, regurgitation, and constipation, were evaluated on a 4-point severity scale. Body weight was measured before and after therapy. A self-rating scale for chewing abilities was filled out after therapy. It was not described whether the GI symptom score and the self-rating scale for chewing abilities were performed during the "on" motor phase. The authors interpreted improved chewing capacity, a moderate gain in body weight, and an improved GI score as signs of improved predigestion.

In the study by Restivo et al. ${ }^{31}$, the effect of percutaneous injected botulinum toxin for cricopharyngeal dysfunction was evaluated in four patients with Parkinson's disease by means of VFS and $\mathrm{EMG}^{30}$. No information was given about the severity of the disease, the 
"on/off" motor phase during measurements, statistics, or whether the rater(s) were blinded to therapy. The method of rating the VFS was not described precisely (visuoperceptual?). The authors concluded that given its safety and effectiveness, the treatment with botulinum neurotoxin type $A$ may be a successful alternative to invasive procedures or may be a useful tool for identifying patients who might benefit from surgical myotomy.

\section{Conclusion}

\section{Methodological problems}

According to the $A B C$ rating scale of Siwek et al. ${ }^{12}$, none of the included articles were level A (randomized controlled trial). Thirteen of the included articles represented level B (nonrandomized clinical trial); however, some of these studies included very small subject populations (e.g., Heckmann et al. ${ }^{29}$ ). Three articles represented level C (expert's opinion). Of the $A B C$ rating scale, level $C$ (expert's opinion/consensus) provides the least evidence for key clinical recommendations. The included studies on the effects of dysphagia treatment in patients with Parkinson's disease varied in quality of design and most of the included studies had at least one methodologic shortcoming that may have introduced bias. In two articles the therapy effects were evaluated positively by the authors despite the fact that only pretreatment examinations were performed ${ }^{19,20}$. These articles provided no evidence of efficacy because of the missing posttherapy measurements.

Some studies did not have an adequate method of randomization for referral to different intervention groups, e.g., Iwasaki et al. ${ }^{23}$ and Bushman et al. ${ }^{27}$ did not score pre- versus posttreatment blinded for the schedule of treatment ${ }^{4,24,26-28}$, nor did they have an adequate placebo or control population ${ }^{7,15,17,22,26-29}$. Very often a control group receiving no therapy for dysphagia when in fact an indication for treatment is present is regarded as unethical and therefore usually not approved by any medical ethical committee. Repeated baseline measurements during the waiting period between the referral for therapy and the actual therapy onset could compensate this shortcoming more or less.

Selection bias might be a methodologic shortcoming in some studies. Despite the fact that the control group had neither a history of dysphagia nor evidence of neurologic or respiratory disease, the use of a rather aged subject population (such as the mean subject's age of 78 years in Pinnington's study ${ }^{16}$ ) makes the presence of dysphagia without subjective complaints more likely.

Calne et al. ${ }^{21}$ described the effect of maximum tolerated doses of L-dopa on the duration of the pharyngeal phase as outcome. However, pretherapy the pharyngeal phase showed normal radiologic patterns. Now it seems as if L-dopa failed to have an effect in an already normal radiologic pattern of deglutition, whereas the information of the total swallowing act was incomplete. In some articles no information was given about the Parkinson's 
disease severity scale $e^{19,21,22,31}$. Some authors did not describe the "on" or "off" motor phase during the examinations and therapy ${ }^{7,19-21,23,29,31}$.

Many studies were unbalanced as a result of small sample sizes. Only seven of the 16 studies described in this review included more than ten subjects (maximum $n=228$ ). Four studies used less than five subjects. Such a small number of subjects restricts the possibility to find statistically significant therapy effects. Furthermore, the justification of the generalization of study outcomes to patients with Parkinson's disease in general or to any other patient population with dysphagia can be questioned. As far as the evaluation tools used to evaluate the therapy effects on dysphagia, the majority of the studies focused on the results found using just one tool ${ }^{4,7,15,17,21-23,27,28}$. As a consequence, certain aspects of the swallowing act may be missed, such as decreased sensory components of swallowing only detectable during FEES(ST) or a patient's subjective evaluation as measured by a quality-of-life questionnaire. In the literature, diverse evaluation instruments have been used to evaluate therapy effects in dysphagia. When using a restricted set of instruments, the information on therapy outcome will be restricted as well. Poorly defined outcome parameters ${ }^{19-21,31}$ and inadequate statistical analysis were also causes of reduced reliability.

In conclusion, because of the diversity in study designs, dysphagia treatment, evaluation techniques, and patient characteristics as well as many methodological problems, statistical pooling of data derived from different articles was not possible.

\section{Therapy effects in literature}

The lack of sufficient evidence of the efficacy of different treatments for dysphagia in Parkinson's disease did not suggest a lack of effect. Summarizing the literature, it can be concluded that positive group tendencies might be observed, although no generalized conclusions can be drawn from these reports.

The literature on the effects of surgical therapy for dysphagia is extremely limited. The outcomes of the studies of the two included articles were described in general positive statements by the authors. These statements were not supported by any statistical proof. The five studies on the rehabilitative treatments for dysphagia provided positive tendencies. It would be interesting to design such studies using large subject populations. The seven articles on pharmacologic treatments had very diverse types and doses of medication. Also, their study designs were too diverse to perform statistical pooling of data. Because of different methodologic problems, large randomized controlled trials on pharmacologic treatment for dysphagia were advised. The therapy effects of other treatments such as implant-prosthodontic treatment and percutaneous injection of botulinum neurotoxin type $A$ in the cricopharyngeal muscle on dysphagia were rather interesting options in the treatment of dysphagia. However, in this field too large welldesigned trials are required. 


\section{Future research}

Recommendations for conducting future therapy trials on the effects of different types of treatment for swallowing disorders in Parkinson's disease include the use of large numbers of patients, adequate placebo therapy if possible, a follow-up period after therapy, notation if patients are "on" or "off" motor phase during measurements and treatment, and using outcome scales validated for dysphagia in subgroups. Nonbiased selection of study participants is necessary. An extended assessment that includes several instruments will provide a more complete view of the complexity of therapy effects in dysphagia. An assessment of therapy results might include a quality-of-life measurement, an evaluation of the nutrition intake, an oral motor function test, a FEES, and/or VFS. Such a multidimensional assessment of the swallowing function pre- and posttherapy is advised because of the complementary aspects of different assessment tools. When using visuoperceptual rating of, for example, VFS recordings, an expert panel should be used, blinded to group of therapy as well as to pre- or posttherapy conditions.

It can be concluded that large randomized controlled trials with a multidimensional swallowing assessment are necessary to assess the effectiveness of the different types of dysphagia treatments in Parkinson's disease. 


\section{References}

1. Ali GN, Wallace KL, Schwartz R, Decarle DJ, Zagami AS, Cook IJ: Mechanisms of oral-pharyngeal dysphagia in patients with Parkinson's disease. Gastroenterology. 1996;110:383-392.

2. Clarke CE, Gullaksen E, Macdonald S, Lowe F: Referral criteria for speech and language therapy assessment of dysphagia caused by idiopathic Parkinson's disease. Acta Neurol Scand. 1998;97:27-35.

3. Coates C, Bakheit AMO: Dysphagia in Parkinson's disease. Eur Neurol. 1997;38:49-52.

4. Nagaya M, Kachi T, Yamada T: Effect of swallowing training on swallowing disorders in Parkinson's disease. Scand J Rehab Med. 2000;32:11-15.

5. Deane KHO, Whurr R, Clarke CE, Playford ED, Ben-shlomo Y: Non-pharmacological therapies for dysphagia in Parkinson's disease (Review). Cochrane Database Syst Rev. 2001;1:CD002816.

6. Robbins J, Gensler G, Hind J, Logemann JA, Lindblad AS, Brandt D, Baum H, Lilienfeld D, Kosek S, Lundy D, Dikeman K, Kazandjian M, Gramigna G, McGarvey-Toler S, Miller Gardner PJ: Comparison of 2 interventions for liquid aspiration on pneumonia incidence. Ann Intern Med. 2008;148:509-518.

7. Logemann JA, Gensler G, Robbins JA, Lindblad AS, Brandt D, Hind JA, Kosek S, Dikeman K, Kazandjian M, Gramigna GD, Lundy D, McGarvey-Toler S, Miller Gardner PJ: A randomized study of three interventions for aspiration of thin liquids in patients with dementia or Parkinson's disease. J Speech Lang Hear Res. 2008; 51:173-183.

8. Burchiel KJ, Anderson VC, Favre J, Hammerstad JP: Comparison of pallidal and subthalamic nucleus deep brain stimulation for advanced Parkinson's disease: results of a randomized, blinded pilot study. Neurosurgery. 1999;45:1375-1384.

9. Bailbé $M$, Bataille B, Paquereau J, Lavazais S, Vandermarcq P, Gil R: Amélioration des troubles de la déglutition par l’apomorphine en perfusion sous cutanée au décours de la mise en place d’électrodes de stimulation cérébrale profonde, dans la maladie de Parkinson. Rev Neurol. 2004;160:352-353.

10. Fonda D, Schwarz J, Clinnick S: Parkinsonian medication one hour before meals improves symptomatic swallowing: a case study. Dysphagia. 1995;10:165-166.

11. Lieberman AN, Horowitz L, Redmond P, Pachter L, Lieberman I, Leibowitz M: Dysphagia in Parkinson's disease. Am J Gastroenterol. 1980;74:157-160.

12. Siwek J, Gourlay ML, Slawson DC, Shaughnessy AF: How to write an evidence-based clinical review article. Am Fam Physician. 2002;65:251-259.

13. Hoehn NM, Yahr MD: Parkinsonism: onset, progression and mortality. Neurology 1967;17:427-442.

14. Wajsbort J: The "off-on" phenomenon during treatment of Parkinson's disease with levodopa. J Neurol. 1997;215:59-66.

15. El Sharkawi A, Ramig L, Logemann JA, Pauloski BR, Rademaker AW, Smith CH, Pawlas A, Baum S, Werner C: Swallowing and voice effects of Lee Silverman Voice Treatment (LSVT): a pilot study. J Neurol Neurosurg Psychiatry. 2002;72:31-36.

16. Pinnington LL, Muhiddin KA, Ellis RE, Playford ED: Non-invasive assessment of swallowing and respiration in Parkinson's disease. J Neurol. 2000;247:773-777.

17. Troche MS, Sapienza CM, Rosenbek JC: Effects of bolus consistency on timing and safety of swallow in patients with Parkinson's disease. Dysphagia. 2008;23:26-32.

18. Rosenbek JC, Robbins JA, Roecker EB, Coyle JL, Wood JL: A penetration-aspiration scale. Dysphagia. 1996;11:93-98.

19. Born LJ, Harned RH, Rikkers LF, Pfeiffer RF, Quigley EM: Cricopharyngeal dysfunction in Parkinson`s disease: Role in dysphagia and response to myotomy. Movement Disorders. 1996;11:53-58.

20. Byrne KG, Pfeiffer R, Quigley EM: Gastrointestinal dysfunction in Parkinson's disease; A report of clinical experience at a single centre. J Clin Gastroenterol. 1994;19:11-16.

21. Calne DB, Shaw DG, Spiers AS, Stern GM: Swallowing in Parkinsonism. Br. J. Radiol. 1970;43:456-457.

22. Hunter PC, Crameri J, Austin S, Woodward MC, Hughes J: Response of parkinsonian swallowing dysfunction to dopaminergic stimulation. J Neurol Neurosurg Psychiatry. 1997;63:579-583.

23. Iwasaki K, Wang Q, Seki H, Satoh K, Takeda A, Arai H, Sasaki H: The effects of the traditional Chinese medicine, "Banxia Houpo Tang (Hange-Koboku To)" on the swallowing reflex in Parkinson's disease. Phytomedicine. 2000;7:259-263.

24. Lim A, Leow L, Huckabee ML, Frampton C, Anderson T: A pilot study of respiration and swallowing integration in Parkinson's disease: "on" and "off" levodopa. Dysphagia. 2008;23:76-81. 
25. McHorney CA: The SWAL-QOL and SWAL-CARE outcomes tool for oropharyngeal dysphagia in adults. III: Documentation of reliability and validity. Dysphagia. 2002;17:97-114.

26. Tison F, Wiart L, Guatterie M, Fouillet N, Lozano V, Henry P, Barat M: Effects of central dopaminergic stimulation by apomorphine on swallowing disorders in Parkinson's disease. Movement Disorders. 1996; 11:729-732.

27. Bushmann M, Dobmeyer SM, Leeker L, Perlmutter JS: Swallowing abnormalities and their response to treatment in Parkinson's disease. Neurology. 1989;39:1309-1314.

28. Fuh JL, Lee RC, Wang SJ, Lin CH, Wang PN, Chiang JH, Liu HC: Swallowing difficulty in Parkinson's disease. Clin Neurol Neurosurg. 1997;99:106-112.

29. Heckmann SM, Heckmann JG, Weber HP: Clinical outcomes of three Parkinson's disease patients treated with mandibular implant overdentures. Clin Oral Impl Res. 2000;11:566-571.

30. Horrowitz M, Harding PE, Chatterton BE, Collins PJ, Shearman DJC. Acute and chronic effects of domperidone on gastric emptying in diabetic autonomic neuropathy. Dig Dis Sci. 1985;30:1-9.

31. Restivo DA, Palmeri A: Botulinum toxin for cricopharyngeal dysfunction in Parkinson's disease. N Engl J Med. 2002;11:1174-1175. 


\section{Chapter 3}

\section{Swallowing in Parkinson patients versus healthy}

controls: reliability of measurements in videofluoroscopy

Laura W.J. Baijens

Renée Speyer

Valéria Lima Passos

Walmari Pilz

Nel Roodenburg

Pere Clavé

Gastroenterol Res Pract 2011;2011:380682
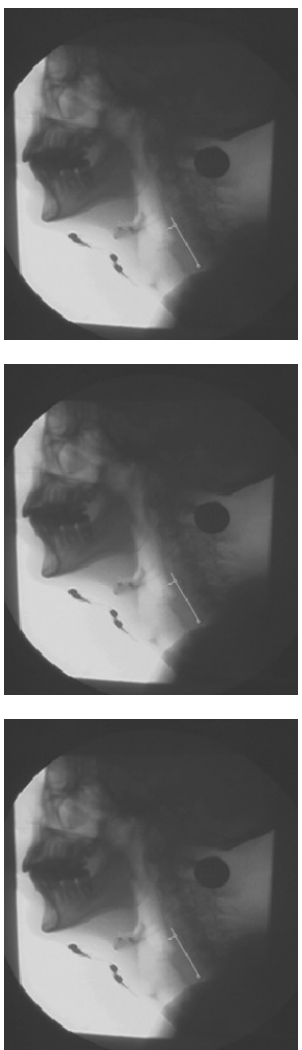


\section{Abstract}

To determine and describe the pathophysiological aspects of oropharyngeal swallowing in patients with Parkinson's disease more accurately, a pilot study of qualitative as well as quantitative parameters of swallowing was performed using videofluoroscopy (VFS). Ten patients with a diagnosis of idiopathic Parkinson's disease having dysphagic complaints and ten healthy age- and gender-matched control subjects underwent a standardized VFS swallowing protocol. Information on the swallowing function was derived from temporal, spatial, and descriptive visuoperceptual parameters. Intra- and interrater reliability was calculated. No significant differences were found between Parkinson patients and healthy control subjects for the majority of the reliable variables. It was concluded that swallowing function seemed to be preserved in the early stages of Parkinson's disease. Furthermore, the reliability of many quantitative as well as qualitative swallowing parameters proved insufficient, raising questions about the interpretation of study outcomes in VFS. 


\section{Introduction}

Dysphagia is a common symptom of Parkinson's disease ${ }^{1}$. In $75-100 \%$ of patients with Parkinson's disease, swallowing abnormalities have been observed during VFS examination $^{2}$. Severe dysphagia can result in malnutrition, dehydration, aspiration pneumonia, and sudden death ${ }^{3}$. Tracheal aspiration is not an uncommon phenomenon in Parkinson patients ${ }^{2,4-6}$. A substantial number of them do not have subjective complaints of dysphagia, despite the presence of abnormalities observed in the swallowing act, including silent aspiration during VFS examination ${ }^{2,6}$. The quality of life decreases as the dysphagia progressively affects oral feeding ${ }^{7,8}$. Previous studies describe oral and pharyngeal phase abnormalities during VFS examination using visuoperceptual parameters, such as piecemeal deglutition, pumping tongue motion, or postswallow vallecular pooling, while others use timed parameters, for example, oropharyngeal transit time, duration of the upper esophageal sphincter opening, or total swallow duration ${ }^{5,9,10}$. There is hardly any literature on the pathophysiology of swallowing in patients with Parkinson's disease using spatial or timed parameters in VFS recordings ${ }^{6}$.

The purpose of this study is twofold: to determine the reliability of timed as well as spatial parameters in VFS necessary to interpret the study outcome and to determine the differences in swallowing function between patients with Parkinson's disease and healthy control subjects, matched for age and gender. Qualitative assessment of swallows was based on visuoperceptual evaluation of VFS signs by an expert panel, whereas quantitative assessment was obtained by means of a specialized computer software application that allowed capturing and digitizing frames in VFS recordings. Using the manual input of anatomical references determined by an expert panel, parameters could be measured.

\section{Methods}

\section{Subject populations}

Patients with idiopathic Parkinson's disease (diagnosed by a neurologist) having subjective clinical complaints of dysphagia were recruited from several neurological departments in the Netherlands. All patients reported dysphagic complaints ranging from mild to severe, for example, these included slow eating, oral or pharyngeal passage disorder, coughing while drinking, and a diminished quality of life as a direct consequence of dysphagia. None of them suffered from any neurological disease except Parkinson's. All patients were able to perform a swallow. Other exclusion criteria were deep brain stimulation (DBS), a Mini Mental State Examination (MMSE) score below 23, severe dyskinesia of head and neck (resulting in problems with VFS recording), mental depression, head and neck cancer, severe cardiopulmological disease, speech therapy during the past six months, or surgery of the swallowing mechanism or the central nervous system ${ }^{11}$. All patients showed a stable disease period at the time of inclusion and had been on the same antiparkinsonian 
medication regimen for more than two months. The clinical severity of the disease was scored using the Hoehn and Yahr $(\mathrm{H}-\mathrm{Y})$ staging scale (Table 3.1) ${ }^{12}$. Healthy control subjects from the department of otorhinolaryngology, head and neck surgery of the university medical center were matched by age and gender. The age difference between the patient and his or her matched control subject was less than four years. The control subjects did not have complaints of swallowing, mental depression, any neurological disease, or head and neck cancer. Nor had they undergone surgery of the swallowing mechanism or the central nervous system. Written informed consent was obtained from all patients and healthy control subjects. This study protocol was approved by the medical ethical committee of the university medical center.

Table $3.1 \quad$ Subject characteristics.

\begin{tabular}{|c|c|c|c|c|c|c|}
\hline \multirow{2}{*}{$\begin{array}{l}\text { Number of } \\
\text { matched pair of } \\
\text { subjects }\end{array}$} & \multirow{2}{*}{$\begin{array}{c}\text { Sex (Female } \\
\text { / Male) }\end{array}$} & \multicolumn{4}{|c|}{ Patients with Parkinson's disease } & \multirow{2}{*}{$\begin{array}{c}\text { Healthy } \\
\text { controls } \\
\text { Age (years) }\end{array}$} \\
\hline & & $\begin{array}{c}\text { Hoehn and } \\
\text { Yahr (H-Y) } \\
\text { scale }^{a}\end{array}$ & $\begin{array}{c}\text { Duration of } \\
\text { Parkinson's disease } \\
\text { (years) }\end{array}$ & $\begin{array}{c}\text { Functional Oral } \\
\text { Intake Scale } \\
\text { (FOIS) }\end{array}$ & $\begin{array}{c}\text { Age } \\
\text { (years) }\end{array}$ & \\
\hline 1 & M & 1 & 5 & 7 & 70 & 68 \\
\hline 2 & $\mathrm{~F}$ & II & 7 & 7 & 64 & 63 \\
\hline 3 & M & II & 7 & 7 & 50 & 46 \\
\hline 4 & M & III & 6 & 7 & 80 & 81 \\
\hline 5 & $\mathrm{~F}$ & II & $>5$ & 7 & 73 & 74 \\
\hline 6 & M & III & 5 & 7 & 57 & 53 \\
\hline 7 & $\mathrm{~F}$ & III & 7 & 7 & 62 & 60 \\
\hline 8 & M & II & 7 & 5 & 70 & 67 \\
\hline 9 & $M$ & III & 16 & 7 & 70 & 70 \\
\hline 10 & $\mathrm{M}$ & 1 & $>5$ & 7 & 66 & 68 \\
\hline
\end{tabular}

${ }^{a} \mathrm{H}-\mathrm{Y}$ scale: the range of scores is one to five, indicating, respectively, unilateral involvement usually with minimal or no functional disability, and confinement to bed or wheelchair unless aided ${ }^{12}$. ${ }^{b}$ FOIS: the range of scores is one to seven, indicating nothing by mouth to total oral diet with no restrictions ${ }^{13}$.

\section{Swallowing assessment}

All patients underwent a detailed clinical examination by an experienced laryngologist and a speech and language pathologist. Before the VFS examination the presence and severity of dysphagia were assessed using the Functional Oral Intake Scale (FOIS) and a fiberoptic endoscopic evaluation of swallowing $(F E E S)^{13,14}$. Following these examinations, all subjects underwent a standardized VFS protocol of three trials of thin liquid (forming part of a more elaborate experiment to be published in a subsequent report). Each trial contained $10 \mathrm{cc}$ of low-density barium ( $40 \% \mathrm{w} / \mathrm{v}$ barium boluses) delivered orally by a syringe. The subjects had to swallow the bolus after it had been accurately delivered by syringe in the oral cavity resulting in a motor challenge without any preparatory cue. The VFS was performed in lateral position. Subjects were seated upright wearing their dental prosthesis (if present). The lips, oral cavity, cervical spine, and proximal cervical esophagus were 
included in the recording. A coin of five eurocents was fixed on the retroauricular skin as a reference distance to correct for magnification. VFS images were obtained with a Philips Diagnost 97 system (Philips Medical Systems, Eindhoven, The Netherlands) and recorded on cassette at 25 frames per second using a mini-DV camera-recorder Panasonic AGDVC30 (Matsushita Electric Industrial Co., Osaka, Japan). If mild aspiration during a trial was observed, a subsequent trial was administered, but in case of severe aspiration the examination was ended. All examinations were performed within 90-120 minutes after the intake of antiparkinsonian medication, thus, during the "on" motor phase ${ }^{15}$. The VFS was performed by an independent experienced speech therapist together with the radiologist.

\section{Measurements}

Swallows were analyzed using a specific software application (Image \& Physiology SL, Barcelona, Spain) to capture, digitize, and measure the VFS sequences ${ }^{16}$. All variables are defined in Table 3.2.

Quantitative measurements were determined for each swallow by two experienced raters with extensive training in the analysis of VFS studies of normal and disordered swallowing. Kahrilas et al. ${ }^{17}$ used more physiologic references to calculate timed variables in contrast with fixed anatomical landmarks, like the point where the mandible crosses the tongue base. In this study, timed variables for biomechanical analysis of swallowing were defined according to Kahrilas et al.: moment of opening and closing of the glossopalatal junction (GPJ); moment of opening and closing of velopharyngeal junction (VPJ); moment of opening and closing of laryngeal vestibule (LV); and moment of opening and closing of upper esophageal sphincter (UES). The frame exhibiting penetration or aspiration was marked by the raters as timed event as well. Penetration was defined as passage of bolus into the laryngeal vestibule. Aspiration was defined as passage of bolus below the level of the vocal folds.

Movement patterns of the hyoid bone as described by Logemann et al. ${ }^{18}$ were used as spatial variables to analyze the swallowing function. For each swallow three points were marked in each video frame: the anterior/superior corner of the hyoid bone and the anterior/inferior corner of the third and fifth cervical vertebral bodies. The $y$-axis was defined by the anterior/inferior corner of the third and fifth cervical vertebral bodies. The $x$-axis crosses the $y$-axis at the anterior/inferior corner of the third vertebral body. By marking these reference points in each frame, movements of the subject in any plane were corrected (Figure 3.1). Software analyzed the extent of movement of the hyoid bone in the $x$ - $y$ coordinate system over time.

The following visuoperceptual parameters to evaluate VFS signs were scored: preswallow anterior and preswallow posterior spill; lingual pumping; swallow hesitancy; piecemeal deglutition; delayed initiation of the pharyngeal reflex; postswallow oral residue; postswallow vallecular pooling; postswallow pyriform sinus pooling ${ }^{7,19}$. Furthermore, the penetration-aspiration (P-A) scale according to Rosenbek et al. ${ }^{20}$ was scored for all VFS 
studies. This eight-point scale (1-8) contains lower scores referring to normal functioning whereas higher scores refer to more severe disability.

Table 3.2 Measurements in videofluoroscopy.

\begin{tabular}{|c|c|c|c|}
\hline \multirow{2}{*}{$\begin{array}{l}\text { Method of } \\
\text { measurement }\end{array}$} & \multicolumn{2}{|l|}{ Variables } & \multirow[b]{2}{*}{$\begin{array}{l}\text { Rating scale } \\
\text { or units }\end{array}$} \\
\hline & Name & Definition & \\
\hline \multirow[t]{16}{*}{$\begin{array}{l}\text { Temporal } \\
\text { variables }\end{array}$} & $\begin{array}{l}\text { GPJo (glossopalatal junction } \\
\text { opening) }\end{array}$ & $\begin{array}{l}\text { Moment of separation of the tongue and soft } \\
\text { palate }\end{array}$ & seconds \\
\hline & $\begin{array}{l}\text { GPJc (glossopalatal junction } \\
\text { closure) }\end{array}$ & $\begin{array}{l}\text { Moment of contact of the tongue and palate } \\
\text { after bolus propulsion }\end{array}$ & \\
\hline & $\begin{array}{l}\text { GPJd (glossopalatal junction } \\
\text { duration) }\end{array}$ & $\Delta T$ between GPJo and GPJc & \\
\hline & $\begin{array}{l}\text { VPJc (velopharyngeal junction } \\
\text { closure) }\end{array}$ & $\begin{array}{l}\text { Moment of first contact of the soft palate } \\
\text { against the posterior pharyngeal wall }\end{array}$ & \\
\hline & $\begin{array}{l}\text { VPJo (velopharyngeal junction } \\
\text { opening) }\end{array}$ & $\begin{array}{l}\text { Moment of separation of the soft palate and the } \\
\text { posterior pharyngeal wall with re-entry of air in } \\
\text { the retrolingual space from the nasopharynx }\end{array}$ & \\
\hline & $\begin{array}{l}\text { VPJd (velopharyngeal junction } \\
\text { duration) }\end{array}$ & $\Delta \mathrm{T}$ between VPJc and VPJo & \\
\hline & $\begin{array}{l}\text { LVc (laryngeal vestibule } \\
\text { closure) }\end{array}$ & $\begin{array}{l}\text { Moment when laryngeal elevation results in } \\
\text { making contact between the arytenoid } \\
\text { cartilages and the underside of the epiglottis }\end{array}$ & \\
\hline & $\begin{array}{l}\text { LVo (laryngeal vestibule } \\
\text { opening) }\end{array}$ & $\begin{array}{l}\text { Moment of separation of the arytenoid } \\
\text { cartilages and the underside of the epiglottis } \\
\text { with re-entry of air in the laryngeal vestibule }\end{array}$ & \\
\hline & $\begin{array}{l}\text { LVd (laryngeal vestibule } \\
\text { duration) }\end{array}$ & $\Delta \mathrm{T}$ between $\mathrm{LVc}$ and $\mathrm{LVo}$ & \\
\hline & $\begin{array}{l}\text { UESo (upper esophageal } \\
\text { sphincter opening) }\end{array}$ & $\begin{array}{l}\text { Moment of opening of the esophagus with entry } \\
\text { of either air or barium }\end{array}$ & \\
\hline & $\begin{array}{l}\text { UESc (upper esophageal } \\
\text { sphincter closure) }\end{array}$ & $\begin{array}{l}\text { Moment of closure of the esophagus after bolus } \\
\text { transport }\end{array}$ & \\
\hline & $\begin{array}{l}\text { UESd (upper esophageal } \\
\text { sphincter duration) }\end{array}$ & $\Delta \mathrm{T}$ between UESo and UESc & \\
\hline & GPJo-LVc & $\Delta \mathrm{T}$ between GPJo and LVc & \\
\hline & GPJo-UESo & $\Delta \mathrm{T}$ between GPJo and UESo & \\
\hline & GPJo-UESC & $\Delta \mathrm{T}$ between GPJo and UESc & \\
\hline & Aspiration-penetration & Moment of aspiration or penetration & \\
\hline \multirow{4}{*}{$\begin{array}{l}\text { Spatial-temporal } \\
\text { variables (hyoid } \\
\text { motion) }\end{array}$} & Horizontal hyoid motion & $\begin{array}{l}\text { Maximum horizontal (anterior) motion during } \\
\text { swallowing act }\end{array}$ & millimeters \\
\hline & Vertical hyoid motion & Maximum vertical motion during swallowing act & millimeters \\
\hline & $\begin{array}{l}\text { Duration vertical hyoid } \\
\text { motion }\end{array}$ & $\begin{array}{l}\text { Duration between initiation of swallow and } \\
\text { moment of maximum vertical motion }\end{array}$ & seconds \\
\hline & $\begin{array}{l}\text { Duration horizontal hyoid } \\
\text { motion }\end{array}$ & $\begin{array}{l}\text { Duration between initiation of swallow and } \\
\text { moment of maximum horizontal (anterior) } \\
\text { motion }\end{array}$ & seconds \\
\hline
\end{tabular}




\begin{tabular}{|c|c|c|c|}
\hline \multirow{2}{*}{$\begin{array}{l}\text { Method of } \\
\text { measurement }\end{array}$} & \multicolumn{2}{|l|}{ Variables } & \multirow[b]{2}{*}{$\begin{array}{l}\text { Rating scale } \\
\text { or units }\end{array}$} \\
\hline & Name & Definition & \\
\hline \multirow[t]{10}{*}{$\begin{array}{l}\text { Visuoperceptual } \\
\text { variables }^{\text {a }}\end{array}$} & Preswallow anterior spill & Preswallow loss of bolus from the lips & $\begin{array}{l}\text { 5-point scale } \\
(0-4)\end{array}$ \\
\hline & Preswallow posterior spill & Preswallow loss of bolus into the pharynx & $\begin{array}{l}\text { 5-point scale } \\
(0-4)\end{array}$ \\
\hline & Lingual pumping & $\begin{array}{l}\text { Preswallow involuntary repetitive tongue } \\
\text { movements }\end{array}$ & $\begin{array}{l}\text { 5-point scale } \\
(0-4)\end{array}$ \\
\hline & Swallow hesitancy & Delayed onset oral transport & $\begin{array}{l}\text { 3-point scale } \\
(0-2)\end{array}$ \\
\hline & Piecemeal deglutition & Sequential swallowing of the same bolus & $\begin{array}{l}\text { 5-point scale } \\
(0-4)\end{array}$ \\
\hline & $\begin{array}{l}\text { Delayed initiation pharyngeal } \\
\text { reflex }\end{array}$ & Delayed onset pharyngeal triggering & $\begin{array}{l}\text { 3-point scale } \\
(0-2)\end{array}$ \\
\hline & Postswallow oral residue & Postswallow pooling in the oral cavity & $\begin{array}{l}\text { 5-point scale } \\
(0-4)\end{array}$ \\
\hline & $\begin{array}{l}\text { Postswallow vallecular } \\
\text { pooling }\end{array}$ & Postswallow pooling in the valleculae & $\begin{array}{l}\text { 3-point scale } \\
(0-2)\end{array}$ \\
\hline & $\begin{array}{l}\text { Postswallow pyriform sinus } \\
\text { pooling }\end{array}$ & Postswallow pooling in the pyriform sinuses & $\begin{array}{l}\text { 3-point scale } \\
(0-2)\end{array}$ \\
\hline & $\begin{array}{l}\text { Penetration-aspiration (P-A) } \\
\text { scale }^{20}\end{array}$ & Penetration and/or aspiration & $\begin{array}{l}\text { 8-point scale } \\
(1-8)\end{array}$ \\
\hline
\end{tabular}

${ }^{a}$ Lower scores refer to normal functioning whereas higher scores refer to more severe disability.

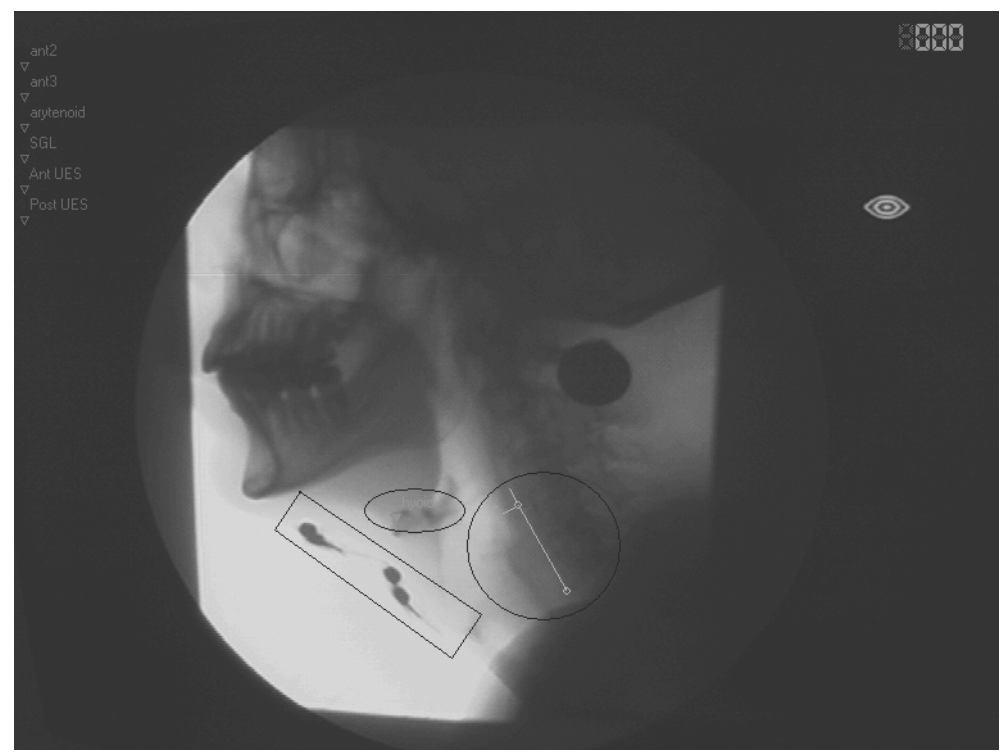

Figure 3.1 Single frame of the videofluoroscopic recording showing the landmarks used for spatial measurements. Extent of movement of the hyoid bone in the $x-y$ coordinate system. The square encloses four electrodes in position during status electrical current "off", the oval includes the marked hyoid bone, and the circle indicates the $x-y$ coordinate system. 
Following consensus training, two experts assessed all quantitative and qualitative variables independently at varying speed, ranging from normal to slow motion to frameby-frame viewing. The raters were blinded to the diagnosis (Parkinson versus healthy subjects) and swallow trials were scored in randomized order. The consensus training in visuoperceptual evaluation was accompanied by a manual including strict, well-defined guidelines to rate these ordinal variables. The exact interpretation per level of each of the three- or five-point scales was trained during five separate sessions with intervals of one week. During these intervals the expert raters had to accomplish test trials separately that were discussed the next session.

To obtain the intrarater reliability each rater performed repeated measurements of all temporal, spatial, and visuoperceptual variables in all swallows within a period of two weeks.

\section{Statistical analysis}

Statistical analysis was conducted stepwise. First, intraclass correlation coefficients (ICC) and Cronbach's $\alpha$ (>0.65) were computed for all quantitative parameters to determine the reliability and the degree of intra- and interrater (absolute) agreement. For all ordinal parameters Cohen's $\boldsymbol{k}$ index of agreement was used. Second, for each reliable temporal, spatial, and visuoperceptual variable, group comparisons were performed over the averaged data of the expert raters. Differences between the Parkinson and the healthy control populations were tested for significance by means of the Mann-Whitney $U$ test (temporal and spatial, continuous variables) or the Chi-Square test (visuoperceptual, ordinal variables). All statistical analyses were performed using SPSS version 16.0 for Windows (SPSS Inc., Chicago, IL, USA).

\section{Results}

\section{Subject populations}

Ten mentally competent dysphagic patients ( 3 women, 7 men) with a diagnosis of idiopathic Parkinson's disease were included. The H-Y staging scale ranged from mild to moderate (median=II). Table 3.1 shows the subject characteristics of all participants. The mean age of the patients and the controls was, respectively, 66 and 65 years. The duration of their Parkinson's disease ranged from 5 to 13 years. The median of the FOIS score in the Parkinson patients was 7. The range of scores of the FOIS is one to seven, indicating nothing by mouth to total oral diet with no restrictions. Subjective complaints of the patients included, for example, extremely slow eating, oral or pharyngeal passage disorder, food stuck in the throat, coughing while drinking, coughing during meals, and a diminished quality of life as a direct consequence of dysphagia. 


\section{Reliability of quantitative and qualitative evaluation of videofluoroscopy}

In Tables 3.3A, 3.3B, and 3.3C the intrarater and interrater reliability of the applied variables is presented. Visuoperceptual variables like preswallow anterior and preswallow posterior spill, swallow hesitancy, and postswallow oral residue mainly scored zero points, meaning normal without any disturbances. It was decided to exclude these variables because of insufficient relevance to this Parkinson patient group. In contrast to the interrater reliability, it proved that the intrarater reliability was sufficient for some variables: delayed initiation pharyngeal reflex; UESd; GPJo-UESc; maximum horizontal (anterior) hyoid motion, for example. When the intrarater reliability proved to be rather low (ICC $<0.60)$, however, the interrater reliability was not computed; this was the case for GPJc, GPJd, UESo, GPJo-UESo, and P-A scale, among others.

Table 3.3A Reliability of the temporal variables (quantitative assessment), intraclass correlation coefficients $(\text { ICC })^{\text {a }}$.

\begin{tabular}{lccc}
\hline Temporal variables & \multicolumn{2}{c}{ Intrarater test-retest reliability ICC } & Interrater reliability ICC \\
\cline { 2 - 3 } & Rater 1 & Rater 2 & - \\
\hline GPJo & - & - & - \\
GPJC & 0.4 & 0.8 & - \\
GPJd & 0.4 & 0.8 & 0.7 \\
VPJc & 0.6 & 0.8 & 0.7 \\
VPJo & 1.0 & 1.0 & 0.7 \\
VPJd & 0.9 & 1.0 & 0.9 \\
LVc & 0.9 & 0.9 & 1.0 \\
LVo & 0.9 & 0.6 & 0.9 \\
LVd & 1.0 & 0.7 & - \\
UESo & 0.4 & 0.5 & 0.8 \\
UESc & 0.6 & 0.8 & -0.1 \\
UESd & 0.7 & 0.8 & 0.9 \\
GPJo-LVc & 0.9 & 0.9 & - \\
GPJo-UESo & 0.4 & 0.9 & -0.02 \\
GPJo-UESc & 0.6 & 0.8 & - \\
Aspiration or penetration & - & - & \\
\hline
\end{tabular}

${ }^{\text {a }}$ Single measures ICC, Cronbach's $\alpha>0.65$

Table 3.3B Reliability of the spatial-temporal variables (hyoid motion), ICC ${ }^{\mathrm{a}}$.

\begin{tabular}{|c|c|c|c|}
\hline \multirow[t]{2}{*}{ Spatial-temporal variables } & \multicolumn{2}{|c|}{$\begin{array}{l}\text { Intrarater test-retest } \\
\text { reliability ICC }\end{array}$} & \multirow[t]{2}{*}{$\begin{array}{l}\text { Interrater } \\
\text { reliability ICC }\end{array}$} \\
\hline & Rater 1 & Rater 2 & \\
\hline Maximum horizontal (anterior) hyoid motion & 0.9 & 0.6 & - \\
\hline Maximum vertical hyoid motion & 0.8 & 0.9 & 0.8 \\
\hline $\begin{array}{l}\text { Duration between initiation of swallow and moment of maximum } \\
\text { horizontal (anterior) hyoid motion }\end{array}$ & 0.9 & 0.9 & 0.9 \\
\hline $\begin{array}{l}\text { Duration between initiation of swallow and moment of maximum } \\
\text { vertical hyoid motion }\end{array}$ & 0.6 & 0.7 & 0.9 \\
\hline
\end{tabular}

\footnotetext{
${ }^{\text {a }}$ Single measures ICC, Cronbach's $\alpha>0.65$
} 
Table 3.3C Reliability of the visuoperceptual variables (qualitative assessment), Cohen's $\kappa$.

\begin{tabular}{|c|c|c|c|}
\hline \multirow[t]{2}{*}{ Visuoperceptual variables } & \multicolumn{2}{|c|}{$\begin{array}{l}\text { Intrarater test-retest reliability } \\
\text { Cohen's } K\end{array}$} & \multirow[t]{2}{*}{$\begin{array}{l}\text { Interrater reliability } \\
\text { Cohen's } K\end{array}$} \\
\hline & Rater 1 & Rater 2 & \\
\hline Preswallow anterior spill & - & - & - \\
\hline Preswallow posterior spill & 1.0 & 1.0 & - \\
\hline Lingual pumping & 1.0 & 1.0 & 0.6 \\
\hline Swallow hesitancy & 1.0 & - & - \\
\hline Piecemeal deglutition & 1.0 & 1.0 & 0.9 \\
\hline Delayed initiation pharyngeal reflex & 0.8 & 1.0 & 0.3 \\
\hline Postswallow oral residue & 0.6 & 0.7 & - \\
\hline Postswallow vallecular pooling & 0.9 & 0.9 & 0.6 \\
\hline Postswallow pyriform sinus pooling & 0.8 & 1.0 & 0.6 \\
\hline Penetration-aspiration scale ${ }^{20}$ & 1.0 & - & - \\
\hline
\end{tabular}

\section{Group differences}

Tables 3.4A, 3.4B, and 3.4C contain descriptive statistics of the reliable temporal, spatial, and visuoperceptual data (ICCs $\geqq 0.60$, Cohen's $k \geqq 0.60$ ). To detect differences in swallowing physiology, data were tested for significant differences between Parkinson patients and the healthy control subjects. Using the Mann-Whitney $U$ test, no significant differences were found regarding the spatial variables. The Chi-Square test did not reveal significant group differences for the visuoperceptual variables. The temporal variable VPJc showed a significant difference between the two groups. Patients with Parkinson's disease showed a significantly delayed velopharyngeal junction closure compared to healthy controls.

Table 3.4A Descriptive statistics of temporal data (quantitative assessment) and level of significance $(P)$ for the comparison of differences between Parkinson patients and the healthy control subjects (MannWhitney $U$ test asymp. sig., 2-tailed).

\begin{tabular}{lccccc}
\hline Temporal variables & \multicolumn{2}{c}{ Parkinson's disease } & \multicolumn{2}{c}{ Healthy controls } & Statistical analyses \\
\cline { 2 - 4 } & Median & $25^{\text {th }}, 75^{\text {th }}$ percentile & Median & $25^{\text {th }}, 75^{\text {th }}$ percentile & \\
\hline VPJc & 0.08 & $0.02,0.12$ & 0.03 & $-0.02,0.06$ & 0.02 \\
VPJo & 0.76 & $0.64,1.00$ & 0.71 & $0.66,0.78$ & 0.31 \\
VPJd & 0.70 & $0.55,0.94$ & 0.66 & $0.64,0.74$ & 0.78 \\
LVc & 0.13 & $0.10,0.26$ & 0.12 & $0.10,0.20$ & 0.58 \\
LVo & 0.91 & $0.72,0.98$ & 0.84 & $0.78,0.91$ & 0.51 \\
LVd & 0.69 & $0.60,0.80$ & 0.66 & $0.60,0.78$ & 0.78 \\
UESc & 0.86 & $0.74,1.14$ & 0.79 & $0.74,0.86$ & 0.13 \\
GPJo-LVc $(\Delta \mathrm{tsec})$ & 0.13 & $0.10,0.26$ & 0.12 & $0.10,0.20$ & 0.58 \\
\hline
\end{tabular}


Table 3.4B Descriptive statistics of spatial-temporal data (hyoid motion) and level of significance $(P)$ for the comparison of differences between Parkinson patients and the healthy control subjects (MannWhitney $U$ test asymp. sig., 2-tailed).

\begin{tabular}{|c|c|c|c|c|c|}
\hline \multirow[t]{2}{*}{ Spatial-temporal variables } & \multicolumn{2}{|c|}{ Parkinson's disease } & \multicolumn{2}{|c|}{ Healthy controls } & \multirow{2}{*}{$\begin{array}{c}\text { Statistical } \\
\text { analyses } \\
P \text { value }\end{array}$} \\
\hline & Median & $25^{\text {th }}, 75^{\text {th }}$ percentile & Median & $\begin{array}{c}25^{\text {th }}, 75^{\text {th }} \\
\text { percentile }\end{array}$ & \\
\hline Maximum vertical hyoid motion (mm) & 13.68 & $9.26,20.02$ & 14.68 & $\begin{array}{l}13.26 \\
19.50\end{array}$ & 0.35 \\
\hline $\begin{array}{l}\text { Duration between initiation of swallow and } \\
\text { moment of maximum anterior/horizontal hyoid } \\
\text { motion }(\Delta \mathrm{t} \mathrm{sec})\end{array}$ & 1.64 & $1.01,2.62$ & 1.12 & $0.86,2.16$ & 0.29 \\
\hline $\begin{array}{l}\text { Duration between initiation of swallow and } \\
\text { moment of maximum vertical hyoid motion } \\
(\Delta t \mathrm{sec})\end{array}$ & 1.72 & $1.04,2.09$ & 1.28 & $0.88,2.04$ & 0.48 \\
\hline
\end{tabular}

Table 3.4C Cross tabulation of visuoperceptual data and level of significance $(P)$ for the comparison of differences between Parkinson patients and the healthy control subjects (Chi-Square test asymp. sig., 2-sided). The number of swallows per level of the three- or five-point scales is in parentheses.

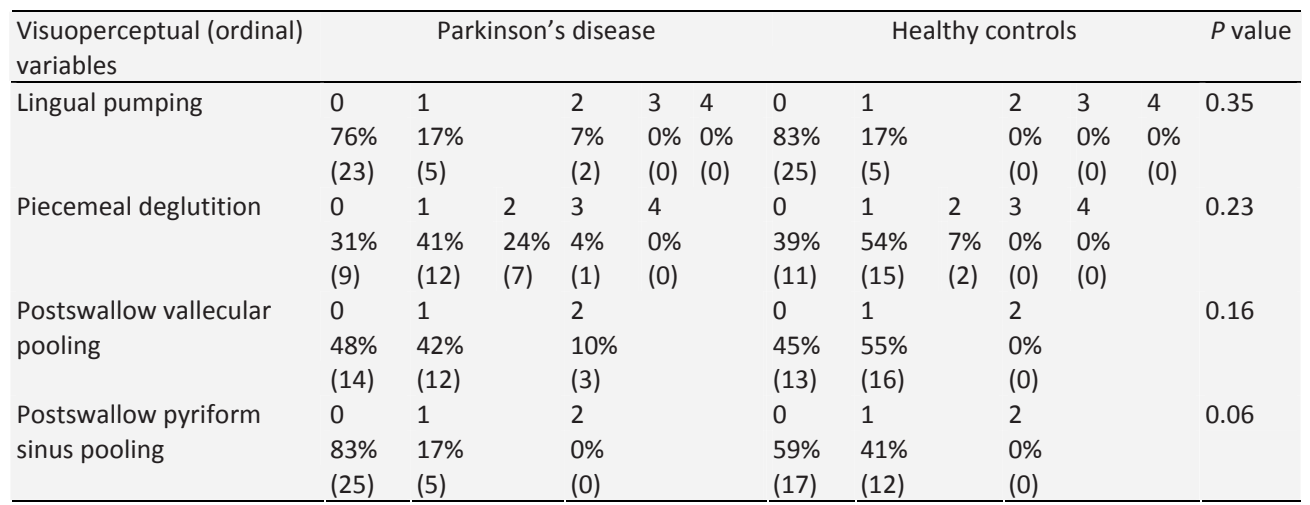

\section{Discussion}

Before interpreting the present study outcome, the intrarater and interrater reliability was determined. Despite the thorough consensus training using well-defined guidelines as well as the strict methodological protocol for repeated measurements by the expert raters to calculate the intra- and interrater reliability in the present study poor reliability was observed for several parameters. Diverse situations may have contributed to this finding. First, despite their high expertise, the raters may have lacked consensus on a definition of, for example, delayed initiation of the pharyngeal reflex or maximum horizontal (anterior) hyoid motion. Second, patients were found to be rather homogeneous with respect to several of the variables being measured; that is, some of them, such as preswallow anterior spill and preswallow posterior spill, always scored zero. Finally, it cannot be excluded that the lack of internal consistency may have resulted from the nature of the 
measurement scales and/or instruments; for instance, visuoperceptual variables were scored on three- or five-point scales. As worldwide no strict guidelines exist, some scope for subjectivity was left to the rater in the discrimination of these ordinal variables. Consequently, these variables were less reliable compared to the temporal or spatial variables that were defined frame by frame using specific software ${ }^{16}$. This software-guided measurement technique, however, is not without its problems either. The fact that a few quantitative variables, for instance GPJo or UESo, were only present or visible in a single frame because of the high speed of movement during the swallowing act may have increased the measurement error and/or the rater's inaccuracy. Furthermore, the method proved to be rather labor-intensive. Finally, fifteen variables could be used for further comparison between Parkinson patients and healthy control subjects.

Despite the presence of a disturbed swallow physiology in Parkinson patients, few significant differences were found between the two pilot groups. Velopharyngeal junction closure (VPJc) was different, being significantly delayed in the Parkinson group. The remaining temporal, spatial, or visuoperceptual variables did not show significant differences or tendencies between the groups. The lack of significant group differences can be the result of the nature and sensitivity of the assessment tool (VFS). As described in the study of Ertekin et al. ${ }^{21}$ conflicting results obtained from radiological and manometric studies in Parkinson patients were found. Also normal motility of the UES region during VFS was observed despite the presence of manometric abnormalities in the same region. In the present pilot study, preservation of the swallowing function during the early stages of Parkinson's disease was found using VFS despite the presence of subjective swallowing complaints in the patients. However, if other assessment tools, for example, manometry might have been used, the study outcome could have been different and abnormalities in swallowing may have been demonstrated. Ertekin et al. ${ }^{21}$ also described some compensatory mechanisms in the course of Parkinson's disease that may explain the benign nature of the deglutition disorder with preservation of swallowing until the terminal stage of the disease. Other explanations for the lack of significance may lie in the advanced age of all subjects (presbyphagia), the rather small sample size, and the moderate severity of Parkinson's disease in the patient population ( $\mathrm{H}-\mathrm{Y}$ scale) ${ }^{5,7}$. For several mainly logistic reasons (e.g., not being able to sit upright for VFS, too weak condition for repeated transport to the outpatient clinic for dysphagia, suffering from Parkinson dementia), patients with severe Parkinson disease who are often admitted to nursing homes did not find easy access to this study. However, the population of included patients was a realistic representation of Parkinson patients consulting a speech therapist for dysphagic complaints. Next, the mechanism of pharyngeal triggering is agedependent $^{22}$. Probably, the natural aging process in the control subjects has been accompanied by altered swallowing function. Furthermore, the presence of electrodes on the skin, which had been used for another study during the same VFS examination, may have worked as tactile cueing in the Parkinson group, even though no electrical stimulation was given ${ }^{23}$. Such cueing may have improved the swallowing performance. 
Finally, only low-density barium boluses were applied. The subjects had to swallow the bolus after it had been accurately delivered by syringe in the oral cavity. It is more difficult for patients with Parkinson's disease to initiate any movement from rest position rather than starting the movement from a previous cueing event or preparatory movement. Seeing this in the light of swallowing, initiating the oral phase is more difficult without any cue or previous movement like the labiobuccal movements or sensorial mucosal stimulation during drinking from a cup or chewing of solid foods than delivery of the bolus by syringe. So, it was decided to apply thin liquid boluses by syringe expecting the highest chance of observing deglutition disorders because of the motor challenge without any preparatory cue. However, other consistencies, such as high-density barium boluses or solid food consistencies, may lead to different dysphagic observations ${ }^{24}$. The discrepancy between symptoms of dysphagia in daily life and radiological results are common in Parkinson's disease, when comparing spontaneous behavior with swallowing under highly controlled experimental conditions ${ }^{25}$. Therefore, the question arises if the FOIS scale is a satisfactory measure for dysphagia severity in this patient population, given the normal scores in the present study.

In order to design therapy effect studies for dysphagia in Parkinson's disease, it is necessary to understand the pathophysiology of swallowing in this patient group. In previous studies, several authors investigated pathological mechanisms of oral-pharyngeal dysphagia in Parkinson patients ${ }^{9,19}$. Several authors suggested rigidity, hypokinesia, and bradykinesia as factors underlying the disordered oral and pharyngeal stages of swallowing ${ }^{6,9}$. Also incomplete cricopharyngeal relaxation, reduced cricopharyngeal opening, and delayed initiation of the swallowing reflex have been suggested as possible mechanisms of dysphagia in this population ${ }^{9,19}$. The authors indicate that parkinsonian patients may be "silent aspirators" with decreased cough reflexes and lack of awareness of aspiration. Abnormal findings in the oral and pharyngeal phase of swallowing were described based on temporal and visuoperceptual variables. Nagaya et al. ${ }^{5}$ observed that durational changes in the pharyngeal phase of swallowing in Parkinson patients were similar to natural changes in elderly control subjects. In the present study differences in swallowing physiology between Parkinson patients and age- and gender-matched healthy control subjects were investigated using qualitative and quantitative variables. These variables to evaluate the oropharyngeal swallowing function in Parkinson's disease were obtained from the very few existing studies on this matter ${ }^{5,6,9,19}$. These studies showed group differences between Parkinson patients and healthy control subjects. However, methodological shortcomings were found in all studies, for example, measurements in the "off" motor phase of Parkinson's disease, only one rater, no consensus training in case of more raters, no blinding, no information on the protocol of repeated measurements, neither on the rating scale of the ordinal variables. So far, the evidence of these studies is neither strong nor reliable enough to serve as comparison for the present study. Despite diverse methodological problems, these studies provided interesting suggestions for variables that could be used in the present study. 
In this pilot study, few restrictions were placed on the kind and number of parameters to use. Per swallow ( $N=60$ swallows in total) thirty variables were assessed, resulting in 1800 measurements. The selection of parameters in follow-up studies should be based on sufficient intra- and interrater reliability as well as on a patient data distribution using the full range of the parameters' scale. The literature on swallowing assessment usually provides only limited information on the definitions of the parameters being used, the exact description of the protocol applied during VFS, the number of raters or the explanation of the concept of an "expert" or "experienced" rater, the intra- and interrater reliability, and the effect of training on reliability coefficients. Interpretation of any study outcome lacking such information may be hazardous. Dysphagic Parkinson patients could potentially benefit from improved diagnostic swallowing assessment by implementing well-defined VFS parameters with sufficient intra- and interrater reliability.

\section{Conclusions}

Despite the special attention paid to methodology in this present study, insufficient reliability for fifteen out of thirty variables (temporal, spatial, and visuoperceptual) was found, particularly for the visuoperceptual variables. The reliable variables could only reveal very few significant differences between both pilot groups. Swallowing seems relatively preserved in the early stages of Parkinson's disease. Recommendations for future research on pathophysiological aspects of swallowing in Parkinson's disease may include study designs with larger numbers of dysphagic patients and healthy control subjects. It may be interesting too to include patients with a more severe degree of Parkinson's disease (higher scores on the H-Y scale) to determine differences between such a patient population and the patients who visit speech therapists as described in this study. The present study has found relatively low intrarater and interrater reliability for many of the variables used despite thorough training and high level of the raters' expertise. In the literature, information on reliability is usually lacking. After training, raters should have maximum consensus about the exact definition of the variables included and be familiar with the rating scales being used as well as with their levels, preferably anchored in detailed descriptions. Any swallowing study should provide information on training and the intrarater and the interrater reliability in order to allow accurate interpretation of the study outcome. Maybe the suggestion that data described in earlier studies may lack sufficient reliability and, therefore, may not be useful in determining therapy outcome, has been rather unexpected. Still, the problems with reliability in outcome studies such as described in this paper may also be the most interesting as well as the most important finding. Newly developed research will need to take this issue into account so that in the near future, in research on the physiology of swallowing in Parkinson's disease, the complementary benefits of using qualitative as well as quantitative variables in VFS can be studied more thoroughly. 


\section{References}

1. Lieberman AN, Horowitz L, Redmond P, Pachter L, Lieberman I, Leibowitz M. Dysphagia in Parkinson's disease. Am J Gastroenterol. 1980;74:157-160.

2. Bird MR, Woodward MC, Gibson EM, Phyland DJ, Fonda D. Asymptomatic swallowing disorders in elderly patients with Parkinson's disease: A description of findings on clinical examination and videofluoroscopy in sixteen patients. Age Ageing. 1994;23:251-254.

3. Marik PE, Kaplan D. Aspiration Pneumonia and dysphagia in the elderly. Chest. 2003;124:328-336.

4. Mari F, Matei M, Ceravolo MG, Pisani A, Montesi A, Provinciali L. Predictive value of clinical indices in detecting aspiration in patients with neurological disorders, J Neurol Neurosurg Psychiatry. 1997;63: 456-460.

5. Nagaya M, Teruhiko K, Yamada T, Igata A. Videofluorographic study of swallowing in Parkinson's disease. Dysphagia. 1998;13:95-100.

6. Robbins JA, Logemann J, Kirshner H. Swallowing and speech production in Parkinson's disease. Ann Neurol. 1986;19:283-287.

7. Ekberg O, Lindgren S, Schultze T. Pharyngeal swallowing in patients with paresis of the recurrent nerve. Acta Radiol Diagn (Stockh). 1986;27:697-700.

8. Leow LP, Huckabee ML, Anderson T, Beckert L. The impact of dysphagia on quality of life in ageing and Parkinson's disease as measured by the swallowing quality of life (SWAL-QOL) questionnaire. Dysphagia. 2010;25:216-220.

9. Nagaya M, Kachi T, Yamada T. Effect of swallowing training on swallowing disorders in Parkinson's disease. Scand J Rehabil Med. 2000;32:11-15.

10. Monte FS, Da Silva-Júnior FP, Braga-Neto P, Nobre-e-Souza M, Sales-de-Bruin VM. Swallowing abnormalities and dyskinesia in Parkinson's disease. Mov Disord. 2005;20:457-462.

11. Folstein MF, Folstein SE, McHugh PR. Mini-mental state. A practical method for grading the cognitive state of patients for the clinician. J Psychiatr Res. 1975;12:189-198.

12. Hoehn NM, Yahr MD. Parkinsonism: onset, progression and mortality. Neurology. 1967;17:427-442.

13. Crary MA, Carnaby Mann GD, Groher ME. Initial psychometric assessment of a Functional Oral Intake Scale for dysphagia in stroke patients. Arch Phys Med Rehabil. 2005;86:1516-1520.

14. Langmore SE, Aviv JE. Endoscopic procedures to evaluate oropharyngeal swallowing. In: Langmore SE, editor. Endoscopic evaluation and treatment of swallowing disorders. New York, Stuttgart: Thieme 2001:73-100.

15. Wajsbort J. The "off-on" phenomenon during treatment of Parkinson's disease with levodopa. J Neurol. 1977;215:59-66.

16. Clavé $P$, de Kraa M, Arreola V, Girvent M, Farré R, Palomera E, Serra-Prat M. The effect of bolus viscosity on swallowing function in neurogenic dysphagia. Aliment Pharmacol Ther. 2006;24:1385-1394.

17. Kahrilas PJ, Lin S, Rademaker A, Logemann J. Impaired deglutitive airway protection: a videofluoroscopic analysis of severity and mechanism. Gastroenterology. 1997;113:1457-1464.

18. Logemann J, Kahrilas PJ, Begelman J, Dodds WJ, Pauloski BR. Interactive computer program for biomechanical analysis of videoradiographic studies of swallowing. AJR Am J Roentgenol. 1989;153: 277-280.

19. Ali GN, Wallace KL, Schwartz R, Decarle DJ, Zagami AS, Cook IJ. Mechanisms of oral-pharyngeal dysphagia in patients with Parkinson's disease. Gastroenterology. 1996;110:383-392.

20. Rosenbek JC, Robbins JA, Roecker EB, Coyle JL, Wood JL. A penetration-aspiration scale. Dysphagia. 1996; 11:93-98.

21. Ertekin C, Tarlaci S, Aydogdu I, Kiylioglu N, Yuceyar N, Turman AB, Secil Y, Esmeli F. Electrophysiological evaluation of Pharyngeal phase of swallowing in patients with Parkinson's disease. Mov Disord. 2002;17: 942-949.

22. Ekberg O, Feinberg MJ. Altered swallowing function in elderly patients without dysphagia: radiologic findings in 56 cases. AJR Am J Roentgenol. 1991;156:1181-1184.

23. Pinnington LL, Muhiddin KA, Ellis RE, Playford ED. Non-invasive assessment of swallowing and respiration in Parkinson's disease. J Neurol. 2000;247:773-777.

24. Troche MS, Sapienza CM, Rosenbek JC. Effects of bolus consistency on timing and safety of swallow in patients with Parkinson's disease. Dysphagia. 2008;23:26-32. 
Chapter 3

25. Fuh JL, Lee RC, Wang SJ, Lin CH, Wang PN, Chiang JH, Liu HC. Swallowing difficulty in Parkinson's disease. Clin Neurol Neurosurg. 1997;99:106-112. 


\section{Chapter 4}

\section{The effect of surface electrical stimulation on}

swallowing in dysphagic Parkinson patients

Laura W.J. Baijens

Renée Speyer

Valéria Lima Passos

Walmari Pilz

Nel Roodenburg

Pere Clavé

Dysphagia 2012;27:528-537
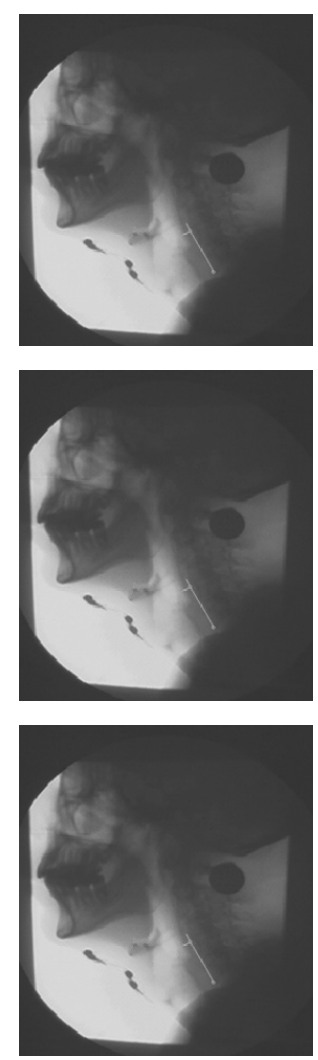


\section{Abstract}

Surface electrical stimulation has been applied on a large scale to treat oropharyngeal dysphagia. Patients suffering from oropharyngeal dysphagia in the presence of Parkinson's disease have been treated with surface electrical stimulation. Because of controversial reports on this treatment, a pilot study was set up. This study describes the effects of a single session of surface electrical stimulation using different electrode positions in ten patients with idiopathic Parkinson's disease (median Hoehn and Yahr score=II) and oropharyngeal dysphagia compared to ten age- and gendermatched healthy control subjects during videofluoroscopy of swallowing (VFS). Three different electrode positions were applied in random order per subject. For each electrode position, the electrical current was respectively turned "on" and "off" in random order. Temporal, spatial, and visuoperceptual variables were scored by experienced raters who were blinded to the group, electrode position, and status (on/off) of the electrical current. Intrarater and interrater reliabilities were calculated. Only a few significant effects of a single session of surface electrical stimulation using different electrode positions in dysphagic Parkinson patients could be observed in this study. Furthermore, significant results for temporal and spatial variables were found regardless of the status of the electrical current in both groups suggesting placebo effects. Following adjustment for electrical current status as well as electrode positions (both not significant, $P>0.05$ ) in the statistical model, significant group differences between Parkinson patients and healthy control subjects emerged. Further studies are necessary to evaluate the potential therapeutic effect and mechanism of electrical stimulation in dysphagic patients with Parkinson's disease. 


\section{Introduction}

The number of reports on the effects of therapies for dysphagia in Parkinson's disease is still small. Reviewing the literature, positive group tendencies may be observed, although no general conclusions can be drawn from these reports ${ }^{1}$. Surface electrical stimulation in dysphagic Parkinson patients has not been investigated in scientific studies before. Carnaby-Mann and Crary $^{2}$ describe a small but significant summary effect size for transcutaneous neuromuscular electrical stimulation (NMES) for dysphagia in a metaanalysis. The immediate effect of surface electrical stimulation on hyolaryngeal movement in normal individuals during swallowing is described by Humbert et al. ${ }^{3}$, who observed a reduced hyolaryngeal elevation during electrical stimulation. They hypothesized a higher risk of further decreased hyolaryngeal elevation during electrical stimulation in dysphagic patients who are already suffering from reduced hyolaryngeal elevation. Ludlow et al. ${ }^{4}$ observed that aspiration and pooling are significantly reduced in chronically dysphagic patients during surface electrical stimulation with low sensory threshold levels of stimulation. Almost all subjects showed depression of the hyoid bone during motor-level stimulation at rest. However, at the maximum motor level of stimulation during swallowing, no group change in aspiration was noted. In a systematic review, Clark et al. ${ }^{5}$ reported promising findings of NMES, emphasizing the need for high-quality controlled trials in the future. Despite the fact that controversial reports have been published ${ }^{3,4}$, surface electrical stimulation is being applied on a large scale for oropharyngeal dysphagia due to diverse neurological etiologies, including Parkinson's disease. The application of surface electrical stimulation in dysphagic patients with Parkinson's disease without existing evidence of therapy effects in the literature has been the rationale for this present study. This study was performed to determine the immediate effect of a single session of surface electrical stimulation using different electrode positions and electrical current states in patients with Parkinson's disease and oropharyngeal dysphagia compared to the same protocol in a group of age- and gender-matched healthy control subjects during a VFS study. The maximum tolerated motor level of stimulation was applied to all subjects during each swallowing act.

\section{Methods}

\section{Patients and healthy control subjects}

Patients with a diagnosis of idiopathic Parkinson's disease and dysphagic complaints were recruited from several neurological departments from diverse hospitals all over the Netherlands. The dysphagic complaints ranged from mild to severe, including slow eating due to a reduced rate of spontaneous swallowing and to rigidity, hypokinesia, and bradykinesia of the masticatory, oral, or pharyngeal musculature involved in swallowing; oral or pharyngeal passage disorder; choking on food; and coughing while drinking or 
eating. The patients continued using their unaltered type and dose of antiparkinsonian medication for at least 2 months and their disease was stable at the time of inclusion. The Hoehn and Yahr $(\mathrm{H}-\mathrm{Y})$ disability score was used as a clinical rating scale to assess the severity of the Parkinson's disease (Table 4.1) ${ }^{6}$. For each patient, an age- and gendermatched control was recruited. The following exclusion criteria were used for the patients as well as for the healthy control subjects: the presence of any other neurological disease; inability to perform a swallow; deep brain stimulation (DBS); a Mini-Mental State Examination (MMSE) ${ }^{7}$ score below 23; severe dyskinesia of head and neck (resulting in problems with VFS recording); mental depression; head and neck cancer; severe cardiopulmonary diseases; speech therapy during the past 6 months; and surgery on the swallowing mechanism or the central nervous system. Written informed consent was obtained from all patients and healthy control subjects. This study protocol was approved by the medical ethical committee of the university medical center.

Table 4.1 Demographics of dysphagic patients with Parkinson's disease and of healthy control subjects.

\begin{tabular}{|c|c|c|c|c|c|}
\hline \multirow{2}{*}{$\begin{array}{l}\text { Number of matched } \\
\text { pair of subjects }\end{array}$} & \multirow{2}{*}{ Sex } & \multirow[t]{2}{*}{ Electrode positions } & \multicolumn{2}{|c|}{ Parkinson's disease patients } & \multirow{2}{*}{$\begin{array}{c}\text { Healthy controls } \\
\text { Age (years) }\end{array}$} \\
\hline & & & $(H-Y)$ scale $^{a}$ & Age (years) & \\
\hline 1 & $M$ & $|-||-|||$ & 1 & 70 & 68 \\
\hline 2 & $\mathrm{~F}$ & III-I-II & II & 64 & 63 \\
\hline 3 & M & $|I-I-I I|$ & II & 50 & 46 \\
\hline 4 & M & III-II-I & III & 80 & 81 \\
\hline 5 & $\mathrm{~F}$ & III-I-II & II & 73 & 74 \\
\hline 6 & M & II-I-III & III & 57 & 53 \\
\hline 7 & $\mathrm{~F}$ & $\mid I I-I I-I$ & III & 62 & 60 \\
\hline 8 & M & $|-||-|||$ & II & 70 & 67 \\
\hline 9 & M & III-I-II & III & 70 & 70 \\
\hline 10 & M & II-I-III & 1 & 66 & 68 \\
\hline
\end{tabular}

${ }^{\mathrm{a}} \mathrm{H}-\mathrm{Y}$ scale = Hoehn and Yahr scale: the range of scores is I-V, where I indicates unilateral involvement, usually with minimal or no functional disability, and a $\mathrm{V}$ indicates confinement to bed or wheelchair unless aided ${ }^{6}$.

\section{Protocol for procedure of surface electrical stimulation}

A two-channel battery-powered electrical stimulator (VitalStim ${ }^{\circledR}$ Therapy; frequency $80 \mathrm{~Hz}$, pulse width $700 \mu \mathrm{s}$, Chattanooga Group, Chattanooga, TN, USA) was used by an experienced laryngologist. The intensity of the bipolar electrode stimulation ranged from 0 to 25 milliamperes $(\mathrm{mA})$ for each channel. All subjects were familiarized with the application of the electrical stimulator before the experiment. All patients and healthy control subjects performed 12 swallows, each containing $10 \mathrm{cc}$ thin liquid barium boluses (low-density barium $40 \% \mathrm{w} / \mathrm{v}$ ). The following single session protocol of electrical stimulation during a standardized VFS examination was performed using three different electrode positions applied in random order per subject. After the skin had been cleaned and shaven, electrodes (VitalStim ${ }^{\circledR}$, reference 59035) were placed on either side of the midline of the neck. In case of hanging skin, a skin lift was performed using medical tape to restore the contact between the skin and the muscle layer. Position I represented two 
electrodes horizontally above the hyoid bone (submental region). Position II represented two electrodes horizontally below the hyoid bone. Position III was a combination of positions I and II with four electrodes connected on each side of the midline of the neck (Figure 4.1).

For each electrode position, the status of the electrical current was three times "on" and once "off", in random order. The VitalStim stimulator cycles automatically off for 1 second every minute. It was ensured that the swallow on VFS with the electrical current turned "on" did not occur during this 1 second "off" period. The maximum tolerated stimulation level resulting in maximum muscle contraction without spasm was applied in accordance with Ludlow et al. ${ }^{4}$. The stimulation intensity was raised gradually in steps of $0.5 \mathrm{~mA}$ until the "grabbing sensation" or maximum tolerated stimulation threshold was reached. Before each swallow, the threshold was reconfirmed to correct for fatigue of the muscles. For this purpose, the stimulation intensity was raised gradually as described before. Each patient and his or her corresponding matched healthy control subject underwent the exact same protocol of electrode positions and electrical current states.
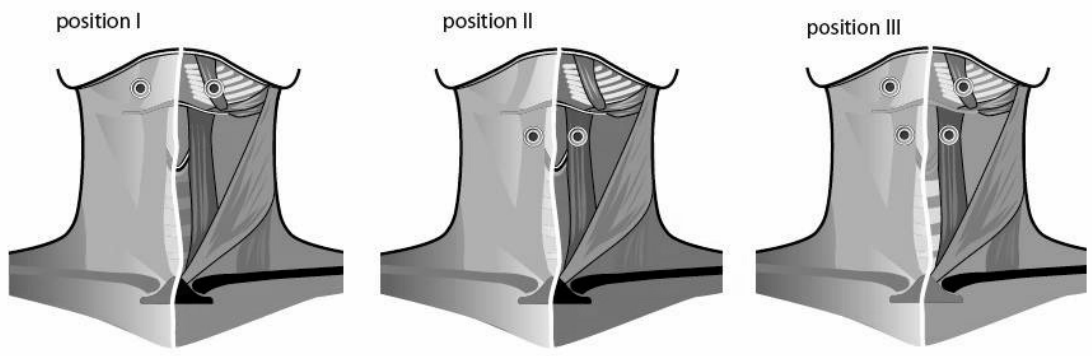

Figure 4.1 Schematic illustration of the electrode positions.

Position I = two electrodes horizontally above the hyoid bone (submental region); position II = two electrodes horizontally below the hyoid bone; position III = combination of positions I and II, with four electrodes connected on each side of the midline of the neck.

\section{Swallowing assessment}

Prior to the experiment, all subjects had a clinical examination by a laryngologist and a speech and language pathologist. The single-session protocol of electrical stimulation was performed during a standardized VFS protocol of 12 trials with thin liquid. Each trial contained $10 \mathrm{cc}$ of low-density barium $(40 \% \mathrm{w} / \mathrm{v})$ delivered orally by a syringe. The subjects had to swallow the bolus after it had been accurately delivered by a syringe in the oral cavity resulting in a motor challenge without any preparatory cue. During the VFS, subjects were seated upright in lateral position wearing their dental prosthesis if present. The field of the VFS image included the lips, oral cavity, cervical spine, and proximal cervical esophagus. A coin of 5 Euro cents was affixed to the retroauricular skin as a 
reference distance to correct for magnification (calibration). VFS images were obtained with a Philips Diagnost 97 system (Philips Medical Systems, Eindhoven, the Netherlands) and recorded on cassette at 25 frames per second using a mini-DV camera-recorder Panasonic AG-DVC30 (Matsushita Electric Industrial Co., Osaka, Japan). Sounds were recorded simultaneously. In the event of mild aspiration during a trial, the examination was continued. If severe aspiration was observed, the examination was ended. All VFS examinations were performed within 90-120 minutes after the intake of antiparkinsonian medication. During this period called the "on" motor phase, the L-dopa motor effect is maximal $^{8}$.

\section{Selection of variables}

For quantitative assessment of the swallow, specialized software was used (Image \& Physiology SL, Barcelona, Spain) to capture, digitize, and measure all swallowing sequences $^{9}$. The capture rate was 25 frames per second. Timed variables were determined for the biomechanical analysis of swallowing ${ }^{10}$. The pharyngeal inlets and outlets were identified frame by frame by both raters during each pharyngeal swallow: moment of opening and closing of the glossopalatal junction (GPJ); moment of opening and closing of the velopharyngeal junction (VPJ); moment of opening and closing of the laryngeal vestibule (LV); and moment of opening and closing of the upper esophageal sphincter (UES). Using definitions of penetration and aspiration according to Rosenbek et al. ${ }^{11}$, the frame exhibiting penetration or aspiration was marked as timed event.

Movement patterns of the hyoid bone were used as spatial variables to analyze the swallowing function quantitatively ${ }^{12}$. For each swallow, three reference points were marked in each video frame: the anterior/superior corner of the hyoid bone and the anterior/inferior corner of the third and fifth cervical vertebral bodies. The $y$-axis was defined by the anterior/inferior corner of the third and fifth cervical vertebral bodies. Perpendicular to the $y$-axis, the $x$-axis crosses at the anterior/inferior corner of the third vertebral body. By marking these reference points in each frame, movements of the subject in any plane could be corrected (Figure 4.2). Next, the extent of movement of the hyoid bone in the $x-y$ coordinate system over time was analyzed by means of specialized software.

Furthermore, visuoperceptual variables were scored for each VFS swallow. The variables are as follows: preswallow anterior and preswallow posterior spill; lingual pumping; swallow hesitancy; piecemeal deglutition; delayed initiation of the pharyngeal reflex; postswallow oral residue; postswallow vallecular pooling; postswallow pyriform sinus pooling; and the penetration-aspiration (P-A) scale of Rosenbek et al. ${ }^{11,13,14}$. In this 8-point scale (1-8), lower scores refer to normal functioning and higher scores refer to more severe disability ${ }^{11}$. Following consensus training, two experts assessed each swallow independently at varying speed, ranging from normal to slow motion to frame-by-frame viewing. The consensus training in visuoperceptual evaluation was accompanied by a 
manual, including strict, well-defined guidelines to rate these ordinal variables. The exact interpretation per level of each of the three- or five-point scales was trained during five separate sessions with intervals of 1 week. During these intervals the expert raters had to accomplish test trials separately that were discussed during the next session.

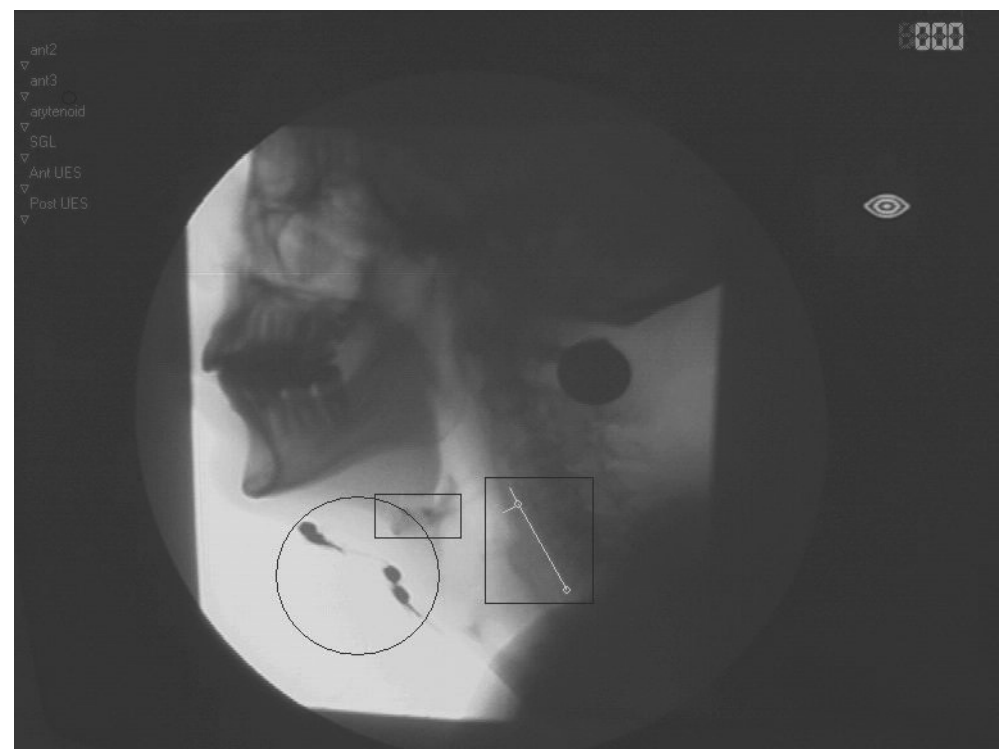

Figure 4.2 Single frame of the videofluoroscopic recording showing the landmarks used for spatial measurements. The circle encloses four electrodes in position, the small rectangle includes the marked hyoid bone, and the large rectangle indicates the $x-y$ coordinate system.

Previous research by Baijens et al. ${ }^{15}$ has described the reliability or intra- and interrater agreement on several quantitative and qualitative parameters in VFS. Only parameters that proved to be sufficiently reliable have been included in the present study (Table 4.2). All measurements and ratings were performed independently by two experienced judges. Both judges were blinded to the group, electrode position, and status of the electrical current (on/off). To optimize blinding to the electrode position during VFS, four electrodes were placed on the skin according to the electrode positions mentioned above. Although the cables were connected to all four electrodes, only the electrodes of the position being studied during a specific swallowing act were stimulated (Figure 4.2). The trials of all subjects were scored in randomized order. Before interpreting the study outcome, the intrarater and interrater reliabilities were determined. To obtain the intrarater reliability, each rater performed repeated measurements of all temporal, spatial, and visuoperceptual variables during the second swallow of each electrode position for all subjects (patients and healthy control subjects) within a period of 2 weeks. 
Table 4.2 Variables with good intrarater and interrater reliability ${ }^{15}$.

\begin{tabular}{|c|c|c|}
\hline $\begin{array}{l}\text { Reliable }^{\text {a }} \text { variables in } \\
\text { videofluoroscopy }\end{array}$ & Name & Definition \\
\hline \multirow[t]{8}{*}{ Temporal variables } & $\begin{array}{l}\text { VPJc (velopharyngeal } \\
\text { junction closure) }\end{array}$ & $\begin{array}{l}\text { Moment of first contact of the soft palate against the } \\
\text { posterior pharyngeal wall (in seconds) }\end{array}$ \\
\hline & $\begin{array}{l}\text { VPJo (velopharyngeal } \\
\text { junction opening) }\end{array}$ & $\begin{array}{l}\text { Moment of separation of the soft palate and the } \\
\text { posterior pharyngeal wall with re-entry of air in the } \\
\text { retrolingual space from the nasopharynx (in seconds) }\end{array}$ \\
\hline & $\begin{array}{l}\text { VPJd (velopharyngeal } \\
\text { junction duration) }\end{array}$ & $\Delta T$ between VPJc and VPJo (in seconds) \\
\hline & $\begin{array}{l}\text { LVc (laryngeal vestibule } \\
\text { closure) }\end{array}$ & $\begin{array}{l}\text { Moment when laryngeal elevation results in making } \\
\text { contact between the arytenoid cartilages and the } \\
\text { underside of the epiglottis (in seconds) }\end{array}$ \\
\hline & $\begin{array}{l}\text { LVo (laryngeal vestibule } \\
\text { opening) }\end{array}$ & $\begin{array}{l}\text { Moment of separation of the arytenoid cartilages and } \\
\text { the underside of the epiglottis with re-entry of air in the } \\
\text { laryngeal vestibule (in seconds) }\end{array}$ \\
\hline & $\begin{array}{l}\text { LVd (laryngeal vestibule } \\
\text { duration) }\end{array}$ & $\Delta T$ between LVc and LVo (in seconds) \\
\hline & $\begin{array}{l}\text { UESc (upper esophageal } \\
\text { sphincter closure) }\end{array}$ & $\begin{array}{l}\text { Moment of closure of the esophagus after bolus } \\
\text { transport (in seconds) }\end{array}$ \\
\hline & $\begin{array}{l}\text { GPJo (glossopalatal junction } \\
\text { opening) -LVc (laryngeal } \\
\text { vestibule closure) }\end{array}$ & $\Delta T$ between GPJo and LVc (in seconds) \\
\hline \multirow{3}{*}{$\begin{array}{l}\text { Spatial-temporal } \\
\text { variables of hyoid motion }\end{array}$} & Vertical hyoid motion & Maximum vertical motion during swallowing act (in $\mathrm{mm}$ ) \\
\hline & $\begin{array}{l}\text { Duration horizontal hyoid } \\
\text { motion }\end{array}$ & $\begin{array}{l}\text { Duration between initiation of swallow and moment of } \\
\text { maximum horizontal (anterior) motion (in seconds) }\end{array}$ \\
\hline & $\begin{array}{l}\text { Duration vertical hyoid } \\
\text { motion }\end{array}$ & $\begin{array}{l}\text { Duration between initiation of swallow and moment of } \\
\text { maximum vertical motion (in seconds) }\end{array}$ \\
\hline \multirow[t]{3}{*}{$\begin{array}{l}\text { Visuoperceptual } \\
\text { variables }^{\text {b }}\end{array}$} & Lingual pumping & $\begin{array}{l}\text { Preswallow involuntary repetitive tongue movements } \\
\text { (5-point scale, } 0-4)\end{array}$ \\
\hline & Piecemeal deglutition & $\begin{array}{l}\text { Sequential swallowing of the same bolus (5-point scale, } \\
0-4)\end{array}$ \\
\hline & $\begin{array}{l}\text { Postswallow vallecular } \\
\text { pooling }\end{array}$ & Postswallow pooling in the valleculae (3-point scale, 0 -2) \\
\hline
\end{tabular}

${ }^{a}$ Cronbach's $\alpha>0.65$ or Cohen's $k$ index of agreement $>0.60, I C C>0.60 .{ }^{b}$ Lower scores refer to normal functioning and higher scores refer to more severe disability.

\section{Statistical analysis}

All temporal and spatial variables had six technical replications for each subject, as these were repeatedly measured in three different electrode positions (suprahyoid, infrahyoid, and bilateral combination) during two electrical current states (on/off), whereas the visuoperceptual variables had 12 technical measurements for each subject. Variables were measured for all electrode positions and electrical current states in both healthy control subjects and Parkinson's patients (Table 4.1). Random intercept models were fit for all reliable VFS outcome parameters. This was done twice: for the patients' only and for all subjects. In the former case, the objective was to determine whether the electrode positions and/or the status of the electrical current affected the VFS outcomes of Parkinson's patients. Patients were defined as random effects, whereas electrode 
positions and status of the electrical current were defined as fixed effects. This model accounts for the correlation induced by the nesting of repeated measurements within subjects. The alternative analyses, using all subjects, aimed at testing additionally for group differences, while concurrently adjusting for electrode position and electrical current status. However, for all subjects the random effect was no longer a single subject but a pair, composed of one patient and his/her matched healthy control (10 pairs in total). This minor change was necessary in order to accommodate dependence induced by matching. As in the case of patients only, the fixed effects consisted of electrode positions and electrical current states, in addition to the group (patient versus control).

Model parameters were estimated via the restricted maximum likelihood method (REML), and parameters were considered statistically significant if the $P$ value of their corresponding likelihood ratio (LR) tests was smaller than 0.05. All statistical analyses were performed using SPSS version 16.0 software (SPSS Inc., Chicago, IL, USA).

\section{Results}

\section{Demographics}

Ten mentally competent dysphagic patients ( 3 women, 7 men) with a diagnosis of idiopathic Parkinson's disease and ten healthy control subjects matched for age and gender were included. The mean age of the patients and the healthy subjects was 66-65 years, respectively, with a maximum age difference of 4 years between patient and matched control. The $\mathrm{H}-\mathrm{Y}$ scale ranged from mild to moderate (median=II). The demographics of the patients and the healthy control subjects are presented in Table 4.1. The patients had their Parkinson's disease from 5 to 13 years.

\section{Reliable variables}

In Table 4.2, all variables with good intrarater and interrater reliabilities are presented (Cronbach's $\alpha>0.65$, Cohen's $\kappa$ index of agreement $>0.60$, and intraclass correlation coefficients $(I C C)>0.60)^{15}$. ICCs and Cronbach's $\alpha$ were computed for all continuous variables. For all ordinal variables, Cohen's $\kappa$ index of agreement was used. Ratings on visuoperceptual ordinal variables like preswallow anterior and preswallow posterior spill, swallow hesitancy, and postswallow oral residue showed no abnormalities. It was decided to exclude these variables because of insufficient relevance to the present Parkinson patient group. The intrarater reliability was not sufficient for the variables delayed initiation pharyngeal reflex, postswallow pyriform sinus pooling, the P-A scale, GPJo, GPJc, and UESo. When the intrarater reliability proved to be rather low (Cohen's $K$ index of agreement $<0.60, \mathrm{ICC}<0.60$ ), the interrater reliability was not computed. 


\section{Effect of electrical stimulation}

Statistical tests for electrical stimulation and/or electrode position did not reveal statistical significance for the vast majority of the VFS parameters in the patient stratum, except for the temporal variable laryngeal vestibule duration (LVd) (Figure 4.3) and the spatiotemporal variable duration horizontal hyoid motion (Figure 4.4). In LVd, time was on average significantly reduced in position II (electrodes are placed horizontally below the hyoid bone) compared to position III (combination of positions I and II), whereas in duration horizontal hyoid motion, time was significantly increased in position II compared to position I (electrodes are placed horizontally above the hyoid bone) (Table 4.3). This same effect was observed for the control group (Figure 4.4). The general lack of significance for electrode positions and electrical current status was strongly confirmed by the random intercept model using all data (patients and healthy control subjects), which showed that these factors did not rise above individual differences. By contrast, once adjusted for electrode position and electrical current status in the statistical model, significant group differences emerged (Table 4.4).

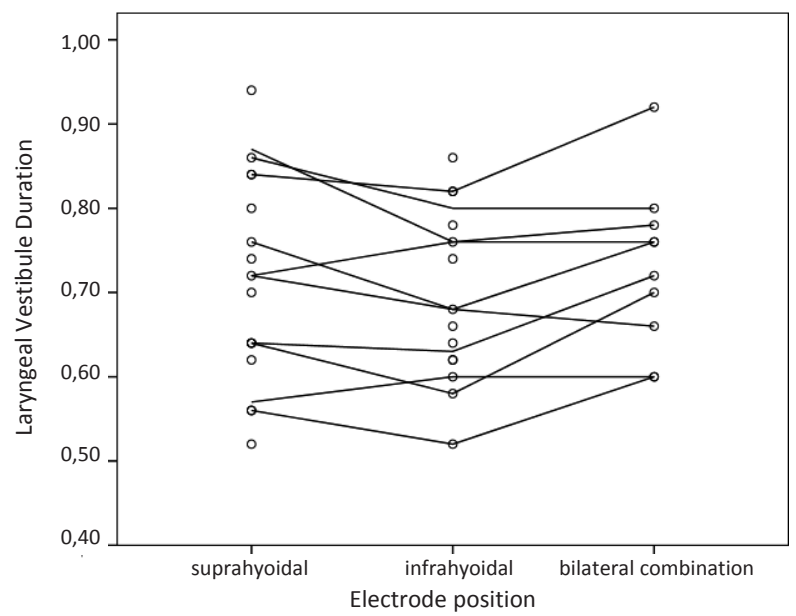

Figure 4.3 Observed mean LVd (laryngeal vestibule duration) values (in seconds) for each patient (individual lines) per electrode position, averaged over the two electrical current states (current on versus off). The dots represent the electrical current status (current on versus off) per patient and per electrode position. The electrode position as a fixed effect, which can be thought of as an average over patients' individual lines, was shown to be significantly reduced in position II compared to position III. 


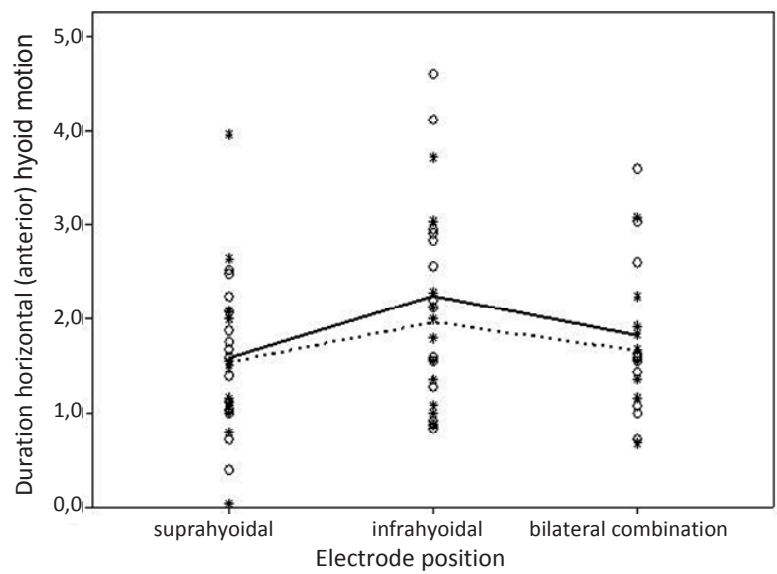

Figure 4.4 Observed values of duration horizontal (anterior) hyoid motion (in seconds), marked for both groups separately (open circle $=$ patients and asterisk = controls, solid and dotted lines, respectively) (all data analysis). Note the marginally higher values for the infrahyoid position (II) for the two groups, detected as statistically significant regardless of the electrical current status (current on versus off). Differences between patients and controls were nonsignificant.

Table 4.3 Random intercept models using patient data.

\begin{tabular}{|c|c|c|c|}
\hline \multirow{2}{*}{$\begin{array}{l}\text { VFS variables } \\
\text { Laryngeal vestibule duration }^{c} \text { (seconds) }\end{array}$} & \multirow{2}{*}{$\begin{array}{c}\text { Electrode position }^{\mathrm{a}} \\
\text { Position I: } \\
0.72(0.03)\end{array}$} & \multicolumn{2}{|c|}{ Comparison between electrode positions ${ }^{b}$} \\
\hline & & $\begin{array}{l}\text { Position I versus II: } \\
0.03(-0.013,0.07)\end{array}$ & NS \\
\hline & $\begin{array}{l}\text { Position II: } \\
0.69(0.03)\end{array}$ & $\begin{array}{l}\text { Position II versus III: } \\
-0.05(-0.097,-0.003)\end{array}$ & Sign. ${ }^{c}$ \\
\hline & $\begin{array}{l}\text { Position III: } \\
0.74(0.03)\end{array}$ & $\begin{array}{l}\text { Position III versus I: } \\
0.022(-0.025,0.069)\end{array}$ & NS \\
\hline \multirow[t]{3}{*}{ Duration horizontal hyoid motion ${ }^{d}$ (seconds) } & $\begin{array}{l}\text { Position I: } \\
1.54(0.27)\end{array}$ & $\begin{array}{l}\text { Position I versus II: } \\
-0.737(-1.288,-0.186)\end{array}$ & Sign. ${ }^{d}$ \\
\hline & $\begin{array}{l}\text { Position II: } \\
2.27(0.28)\end{array}$ & $\begin{array}{l}\text { Position II versus III: } \\
0.488(-0.139,1.116)\end{array}$ & NS \\
\hline & $\begin{array}{l}\text { Position III: } \\
1.78(0.33)\end{array}$ & $\begin{array}{l}\text { Position III versus I: } \\
0.248(-0.379,0.876)\end{array}$ & NS \\
\hline
\end{tabular}

Significant VFS variables and the estimated means plus standard errors per electrode position and the mean differences between electrode positions in combination with the 95\% confidence intervals (according to the fitted random intercept models). These VFS variables show significant differences for electrode position regardless of the electrical current status (current on versus off). ${ }^{a}$ Values are mean (standard error). ${ }^{b}$ Values are mean difference $(95 \% \mathrm{CI}) .{ }^{c}$ Statistically significant pairwise difference between II and III. ${ }^{\mathrm{d}}$ Statistically significant pairwise difference between I and II.

In Figure 4.5, for instance, box plots of the temporal variable UESc of the individual matched pairs are displayed. The objective of this illustration is two-fold. First, it allows for a visualization of all data (patients and healthy control subjects), highlighting the nesting of the subjects within pairs. Second, it shows that for six out of the ten patient-control pairs, the patients had values that are larger than or comparable to those for the controls, 
resulting in the statistically significant difference observed between the two groups. The significant results of the random intercept models using patient data and using all data (patients and healthy control subjects) are summarized in Tables 4.3 and 4.4, where the marginal estimated mean values for the significant fixed effects are given (the current on/off effect is omitted due to its nonsignificance).

Table 4.4 Random intercept models using all data (patients and healthy control subjects).

\begin{tabular}{llcc}
\hline VFS variables & Group & $\begin{array}{c}\text { Mean } \\
\text { (standard error) }\end{array}$ & $\begin{array}{c}\text { Mean difference between } \\
\text { patients and controls }(95 \% \mathrm{Cl})\end{array}$ \\
\hline Velopharyngeal junction closure (seconds) & $\begin{array}{l}\text { Patients } \\
\text { Controls }\end{array}$ & $\begin{array}{r}0.054(0.03) \\
-0.005(0.03)\end{array}$ & $0.059(0.002,0.117)$ \\
Laryngeal vestibule closure (seconds) & $\begin{array}{l}\text { Patients } \\
\text { Controls }\end{array}$ & $0.16(0.06)$ & $0.152(0.024,0.277)$ \\
Laryngeal vestibule duration (seconds) & Patients & $0.73(0.05)$ & $-0.32(-0.642,-0.003)$ \\
Upper esophageal sphincter closure (seconds) & Controls & $1.05(0.05)$ & \\
& Patients & $0.87(0.04)$ & $0.07(0.001,0.127)$ \\
Piecemeal deglutition (5-point scale, 0-4) & Controls & $0.80(0.04)$ & \\
& Patients & $1.248(0.17)$ & $0.557(0.235,0.878)$ \\
\hline
\end{tabular}

Significant VFS variables and their estimated means plus standard errors for patients and controls separately, in combination with the $95 \% \mathrm{Cl}$ of the mean difference between both groups. In the random intercept model, the data have been adjusted for electrode position and electrical current status (current on versus off) which are both not significant.

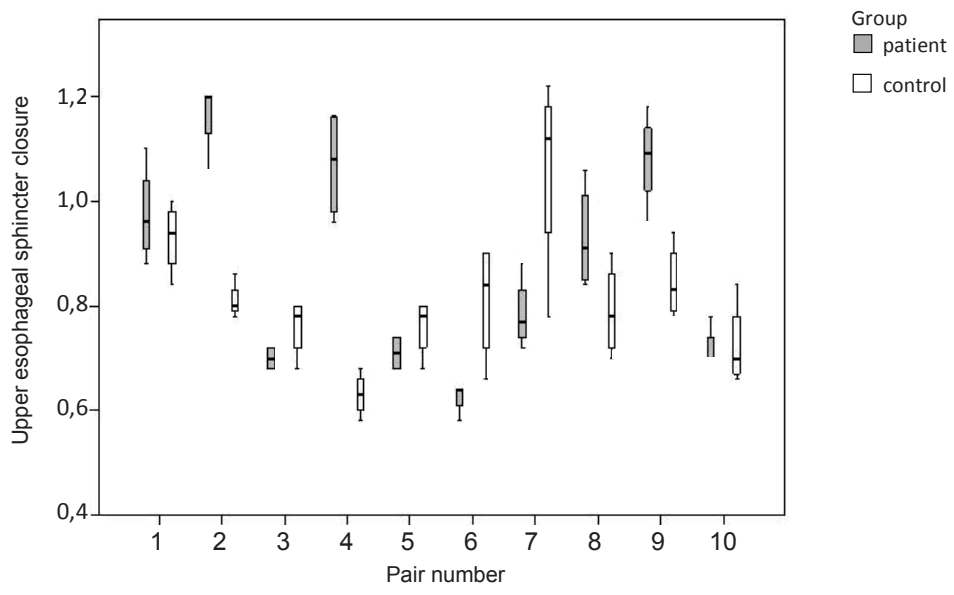

Figure 4.5 Box plots of UESc (upper esophageal sphincter closure in seconds) showing the nesting of subjects within each matched patient-control pair. For UESc a statistically significant difference is observed between the two groups after adjustment for electrode position and electrical current status (current on versus off) which are both not significant. 


\section{Discussion}

Studying therapy effects for dysphagia in Parkinson's disease using surface electrical stimulation is a challenge. In previous studies, several authors have investigated the effects of surface electrical stimulation in other populations such as chronically dysphagic patients, stroke patients, or healthy subjects ${ }^{3,4,16}$. However, the pathological mechanisms of oropharyngeal dysphagia in Parkinson patients are quite different from the mechanisms of chronic dysphagia in many other diseases, e.g., dysphagia following treatment for head and neck cancer or stroke. The pathophysiology of dysphagia in Parkinson's disease is complex and dependent on the coordination among both peripheral and central nervous system factors; uncoordinated or disrupted signals along these pathways (dopaminergic and nondopaminergic) can lead to oropharyngeal dysphagia. Several investigators have designed therapy-effect studies based on treatment of dysphagic features that are considered specific to Parkinson's disease, including rigidity, hypokinesia, and bradykinesia of the oral or pharyngeal stages of swallowing, incomplete cricopharyngeal relaxation, reduced cricopharyngeal opening and delayed initiation of the swallowing reflex ${ }^{17-19}$. Therapies aimed at reduction of the aspiration risk in Parkinson's disease can include voluntary airway protection techniques and bolus modification ${ }^{20-22}$.

In the present study, differences in swallowing physiology during a single-session protocol of surface electrical stimulation were investigated in Parkinson patients and age- and gender-matched healthy control subjects using qualitative and quantitative variables in VFS that have proved to be sufficiently reliable (Table 4.2).

Poor reliability was observed for the P-A scale, among others variables. Diverse situations may have contributed to this finding. First, despite their high level of expertise, the strict methodological protocol for repeated measurements, and the consensus training, the raters may have lacked consensus on a definition of the levels of the scale. Second, patients were found to be rather homogeneous with respect to several of the variables being measured, i.e., some variables such as preswallow anterior spill, preswallow posterior spill, and the P-A scale almost always scored consistently the same level of the ordinal scale.

For most temporal, spatial, and visuoperceptual variables, no statistically significant changes were found during surface electrical stimulation. For the temporal variable laryngeal vestibule duration (LVd), a significant effect was found for position II (in which the electrodes are placed horizontally below the hyoid bone) compared to position III (combination of positions I and II) regardless of the status of the electrical current in the Parkinson and healthy control groups, possibly indicating a placebo effect. The value of this temporal measurement is significantly reduced in position II compared to position III, resulting in a decreased period of laryngeal closure. Although the risk of penetration or even aspiration may not be increased in the present Parkinson population, it may be increased in patients with more severe Parkinson disease due to a decreased period of laryngeal closure. For the spatially related temporal variable duration horizontal hyoid motion, a significant effect is found for the electrode position II compared to position I 
(electrodes are placed horizontally above the hyoid bone), again regardless of the status of the electrical current in Parkinson patients and healthy control subjects. The value of this variable increases significantly in position II compared to position I, indicative of the hyoid motion being slowed down.

It is known that several therapies induce a placebo effect in Parkinson patients and in healthy volunteers ${ }^{23,24}$. The presence of skin electrodes and the connection of the electrical stimulation device may have worked as a sham intervention. The lack of a significant effect of surface electrical stimulation in the present study may not be a simple issue of statistical power. Continuous and ordinal variables were assessed per swallow ( $N=240$ swallows in total), resulting in 4800 measurements. It was expected that this number of swallows and variables would reveal significant differences or at least indicate at possible tendencies in measurement data.

The fixed stimulation variables (frequency $=80 \mathrm{~Hz}$, pulse width $=700 \mu \mathrm{s}$, current intensity $=0-25 \mathrm{~mA}$ ) of the VitalStim electrical stimulator may not have been optimal for inducing any effects during the applied single-session protocol of surface electrical stimulation in Parkinson's patients. Oropharyngeal excitability depends on the stimulation variables ${ }^{25}$. Therefore, different values of the stimulation variables may cause different effects in Parkinson's disease. Oropharyngeal dysphagia in the presence of Parkinson's disease can be due to dysfunction of central nervous system pathways of swallowing rather than muscle weakness or peripheral sensory dysfunction for which the applied electrical stimulation device was mainly designed for ${ }^{26,27}$. The possibility that snap skin electrodes may not be a selective technique of neuromuscular stimulation cannot be disregarded too. It is likely that muscles or other tissues not involved in swallowing physiology or antagonistic muscles (muscles that pull the hyoid bone upward or downward) have been stimulated simultaneously, thereby not resulting in a measurable effect in $\mathrm{VFS}^{4}$. Synchronous electromyographic (EMG) feedback of the supra- and infrahyoid muscles was not performed to exclude this phenomenon. Intramuscular stimulation as described by Burnett et al. ${ }^{28}$ provides a more precise technique of electrical stimulation, although many patients may not be willing to accept invasive electrodes. In addition to these explanations, the lack of significant effects may also be caused by the choice of the assessment tools as well as the outcome variables. VFS may not be the most optimal assessment tool to indicate possible effects of surface electrical stimulation in dysphagic Parkinson patients. Ertekin et al. ${ }^{26}$ described conflicting results of radiological studies. Some of those studies did not detect any abnormalities in the swallowing of Parkinson patients, unlike other studies that found abnormalities in similar populations of Parkinson patients $^{26}$. Furthermore, normal motility of the UES region during VFS was observed despite the presence of manometric abnormalities in the same region ${ }^{26,29}$. Other assessment tools such as EMG or manometry may be more sensitive and reveal significant effects $^{26}$. However, as the assessment protocol was already a burden on the patients, it was decided not to subject them to additional assessment tools.

Another reason for finding few significant results from the use of surface electrical stimulation can be the relatively intact swallow physiology in the early phase of 
Parkinson's disease as observed in this pilot population. The population of included patients is a realistic representation of Parkinson patients who consult their speech therapists about dysphagic complaints. For several mainly logistic reasons (e.g., not being able to sit upright for VFS, condition too weak for repeated transport to the outpatient clinic for dysphagia, and suffering from Parkinson dementia), patients with severe Parkinson's disease, who are often admitted to nursing homes, did not find easy access to this study. On the other hand, following adjustment for electrode position and electrical current status in the random intercept models (Table 4.4), several variables show significant group differences (patients versus healthy control group) despite the early phase of Parkinson's disease. These group differences may be a result of pathophysiological changes in swallowing due to Parkinson's disease after all. Several temporal variables show significant differences: moment of velopharyngeal junction closure, moment of laryngeal vestibule closure, and moment of upper esophageal sphincter closure appear significantly later in the swallow of Parkinson patients compared to that of healthy control subjects. As stated earlier, rigidity, hypokinesia, and bradykinesia of the oral or pharyngeal stage of swallowing in Parkinson's disease may be the reason for these significant differences ${ }^{17-19}$. The duration of the laryngeal vestibule closure (laryngeal vestibule duration) is significantly shorter in Parkinson patients compared to healthy control subjects. This phenomenon may result in a less protected airway in Parkinson patients $^{30}$. The visuoperceptual variable piecemeal deglutition (sequential swallowing of the same bolus) has a significant higher score in Parkinson patients compared to healthy control subjects. Parkinson patients need more swallows for the same liquid bolus before the entire bolus has entered the esophagus. Possible explanations are oropharyngeal muscle weakness, peripheral sensory dysfunction, or central nervous system dysfunction in Parkinson's disease resulting in less efficient bolus formation, delayed oropharyngeal bolus transition, and aspiration ${ }^{26,31}$.

\section{Conclusion}

A single session of surface electrical stimulation using different electrode positions in dysphagic Parkinson patients during VFS resulted in only a few statistically significant effects. The present study provides preliminary data which shows that surface electrical stimulation can alter swallowing in Parkinson's disease. However, these effects may have been caused mainly by the placebo effect. The changes measured from surface electrical stimulation were found in both healthy control subjects and Parkinson patients and the direction of change would not likely benefit swallowing. Questions arise about the mechanism of surface electrical stimulation and its effects on the neural pathways involved in swallowing. Following adjustment in the statistical model for electrical current status as well as electrode positions (both not significant), additional information appeared on group differences between Parkinson patients and healthy control subjects for several variables. 
Further studies using, for example, larger patient populations, a sham intervention group, different electrical stimulation variables (frequency, amplitude, and pulse width) or EMG feedback are necessary to evaluate the potential therapeutic effect of electrical stimulation in dysphagic patients with Parkinson's disease. Furthermore, as stated in an earlier study by Baijens et al. ${ }^{15}$, the intrarater and interrater reliabilities in outcome studies such as this one and in future research remains a major concern. In order to determine the effects of therapy interventions on swallowing and to compare study outcomes for different subject populations, measurement protocols must be standardized and based on reproducible, reliable ratings of well-defined variables. 


\section{References}

1. Baijens LWJ, Speyer R. Effects of therapy for dysphagia in Parkinson's disease. Systematic review. Dysphagia. 2009;24:91-102.

2. Carnaby-Mann GD, Crary MA. Examining the evidence on neuromuscular electrical stimulation for swallowing: a meta-analysis. Arch Otolaryngol Head Neck Surg. 2007;133:564-571.

3. Humbert I, Poletto C, Saxon K, Kearney PR, Crujido L, Wright-Harp W, Payne J, Jeffries N, Sonies B, Ludlow $\mathrm{CL}$. The effect of surface electrical stimulation on hyo-laryngeal movement in normal individuals at rest and during swallowing. J Appl Physiol. 2006;101:1657-1663.

4. Ludlow CL, Humbert I, Saxon K, Poletto C, Sonies B, Crujido L. Effects of surface electrical stimulation both at rest and during swallowing in chronic pharyngeal dysphagia. Dysphagia. 2007;22:1-10.

5. Clark H, Lazarus C, Arvedson J, Schooling T, Frymark T. Evidence-based systematic review: effects of neuromuscular electrical stimulation on swallowing and neural activation. Am J Speech Lang Pathol. 2009; 18:361-375.

6. Hoehn NM, Yahr MD. Parkinsonism: onset, progression and mortality. Neurology. 1967;17:427-442.

7. Folstein MF, Folstein SE, McHugh PR. "Mini-mental state". A practical method for grading the cognitive state of patients for the clinician. J Psychiatr Res. 1975;12:189-198.

8. Wajsbort J. The "off-on" phenomenon during treatment of Parkinson's disease with levodopa. J Neurol. 1977;215:59-66.

9. Clavé P, de Kraa M, Arreola V, Girvent M, Farré R, Palomera E, Serra-Prat M. The effect of bolus viscosity on swallowing function in neurogenic dysphagia. Aliment Pharmacol Ther. 2006;24:1385-1394.

10. Kahrilas PJ, Lin S, Rademaker A, Logemann J. Impaired deglutitive airway protection: a videofluoroscopic analysis of severity and mechanism. Gastroenterology. 1997;113:1457-1464.

11. Rosenbek JC, Robbins JA, Roecker EB, Coyle JL, Wood JL. A penetration-aspiration scale. Dysphagia. 1996; 11: 93-98.

12. Logemann J, Kahrilas PJ, Begelman J, Dodds WJ, Pauloski BR. Interactive computer program for biomechanical analysis of videoradiographic studies of swallowing. AJR Am J Roentgenol. 1989;153: 277-280.

13. Robbins JA, Logemann J, Kirshner H. Swallowing and speech production in Parkinson's disease. Ann Neurol. 1986;19:283-287.

14. Nagaya M, Teruhiko K, Yamada T, Igata A. Videofluorographic study of swallowing in Parkinson's disease. Dysphagia. 1998;13:95-100.

15. Baijens L, Speyer R, Lima Passos V, Pilz W, Roodenburg N, Clavé P. Swallowing in Parkinson patients versus healthy controls: Reliability of measurements in videofluoroscopy. Gastroenterol Res Pract. 2011;2011:380682.

16. Bülow M, Speyer R, Baijens L, Woisard V, Ekberg O. Neuromuscular electrical stimulation (NMES) in stroke patients with oral and pharyngeal dysfunction. Dysphagia. 2008;23:302-309.

17. Pinnington LL, Muhiddin KA, Ellis RE, Playford ED. Non-invasive assessment of swallowing and respiration in Parkinson's disease. J Neurol. 2000;47:773-777.

18. Lim A, Leow L, Huckabee ML, Frampton C, Anderson T. A pilot study of respiration and swallowing integration in Parkinson's disease: "on" and "off" levodopa. Dysphagia. 2008;23:76-81.

19. Nagaya M, Kachi T, Yamada T. Effect of swallowing training on swallowing disorders in Parkinson's disease. Scand J Rehab Med. 2000;32:11-15.

20. Bushmann M, Dobmeyer SM, Leeker L, Perlmutter JS. Swallowing abnormalities and their response to treatment in Parkinson's disease. Neurology. 1989;39:1309-1314.

21. Troche MS, Sapienza CM, Rosenbek JC. Effects of bolus consistency on timing and safety of swallow in patients with Parkinson's disease. Dysphagia. 2008;23:26-32.

22. Logemann JA, Gensler G, Robbins J, Lindblad AS, Brandt D, Hind JA, Kosek S, Dikeman K, Kazanjian M, Gramigna GD, Lundy D, McGarvey-Toler S, Miller Gardner PJ. A randomized study of three interventions for aspiration of thin liquids in patients with dementia or Parkinson's disease. J Speech Lang Hear Res. 2008;51: 173-183.

23. Pollo A, Benedetti F. The placebo response: neurobiological and clinical issues of neurological relevance. Prog Brain Res. 2009;175:283-294. 
24. Rosenzweig P, Brohier S, Zipfel A. The placebo effect in healthy volunteers: influence of experimental conditions on the adverse events profile during phase I studies. Clin Pharmacol Ther. 1993;54:578-583.

25. Fraser C, Rothwell J, Power M, Hobson A, Thompson D, Hamdy S. Differential changes in human pharyngoesophageal motor excitability induced by swallowing, pharyngeal stimulation, and anesthesia. Am J Physiol Gastrointest Liver Physiol. 2003;285:G137-144.

26. Ertekin C, Tarlaci S, Aydogdu I, Kiylioglu N, Yuceyar N, Turman AB, Secil Y, Esmeli F. Electrophysiological evaluation of pharyngeal phase of swallowing in patients with Parkinson's disease. Mov. Disord. 2002;17: 942-949.

27. Freed M, Wijting Y. VitalStim Certification Program. Training manual for patient assessment and treatment using VitalStim electrical stimulation. Hixson TN: Chattanooga Group; 2003.

28. Burnett TA, Mann EA, Cornell SA, Ludlow CL. Laryngeal elevation achieved by neuromuscular stimulation at rest. J Appl Physiol. 2003;94:128-134.

29. Ali GN, Wallace KL, Schwartz R, DeCarle DJ, Zagami AS, Cook IJ. Mechanisms of oral-pharyngeal dysphagia in patients with Parkinson's disease. Gastroenterology 1996;110:383-392.

30. Kendall KA, Leonard RJ. Bolus transit and airway protection coordination in older dysphagic patients. Laryngoscope. 2001;111:2017-2021.

31. Troche MS, Okun MS, Rosenbek JC, Musson N, Fernandez HH, Rodriguez R, Romrell J, Pitts T, WheelerHegland KM, Sapienza CM. Aspiration and swallowing in Parkinson disease and rehabilitation with EMST: a randomized trial. Neurology. 2010;23:1912-1919. 


\section{Chapter 5}

Surface electrical stimulation in dysphagic Parkinson patients: a randomized clinical trial

Laura W.J. Baijens

Renée Speyer

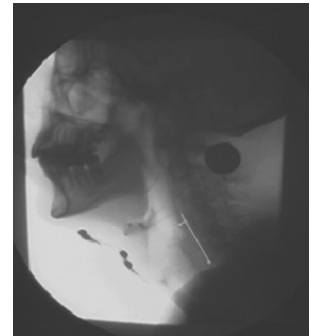

Valéria Lima Passos

Walmari Pilz

Jolien van der Kruis

Saskia Haarmans

Christel Desjardins-Rombouts

The Laryngoscope 2013;123:E38-44
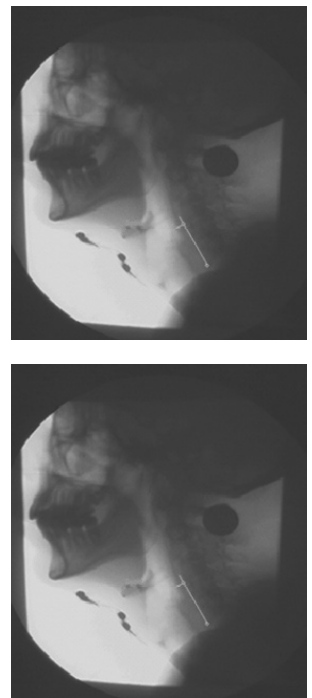


\section{Abstract}

A new treatment for oropharyngeal dysphagia in Parkinson's disease was evaluated in the present prospective randomized controlled trial.

The study describes the effects of surface electrical stimulation (SES) of the neck (submental region) in dysphagic Parkinson patients using different intensities of electrical current. Quasi-random allocation was performed when assigning patients to treatment groups. Three groups consisting of dysphagic patients with idiopathic Parkinson's disease $(\mathrm{N}=90)$ received daily treatment for 15 days with periods of no treatment during the weekend. All three received traditional logopedic dysphagia treatment. In addition, two groups received SES, either motor-level or sensory-level stimulation. A standardized measurement protocol, including fiberoptic endoscopic evaluation of swallowing (FEES) and videofluoroscopy of swallowing (VFS), was performed before and after therapy. A team of experienced raters was blinded to the treatment group and to the moment of measurement. Intrarater and interrater reliabilities were calculated.

Using proportional odds models (POMs), some of the visuoperceptual ordinal outcome variables showed significant improvement in all groups following treatment. Following 15 days of SES of the submental region, few significant effects were found, suggesting a therapy effect of traditional logopedic dysphagia treatment without any additional influence of SES.

On the grounds of this study, it is concluded that further research is needed on the exact mechanism of SES and its effects on the neural pathways involved in swallowing. 


\section{Introduction}

Dysphagia treatment in patients with Parkinson's disease remains a challenge. The increased prevalence of Parkinson's disease in an aging population underlines the need for further research ${ }^{1}$. Initial treatment for oropharyngeal dysphagia in Parkinson's disease can be the administration of antiparkinsonian medication like L-dopa ${ }^{2,3}$. In case of persistent dysphagic symptoms despite pharmacological treatment, however, alternative avenues such as logopedic or surgical treatment can be taken into consideration, depending on the dysphagic findings and disease severity ${ }^{3}$. As yet, no investigation has been done on a rehabilitation program for oropharyngeal dysphagia using surface electrical stimulation (SES) of the neck combined with traditional logopedic dysphagia treatment in Parkinson patients. The number of studies evaluating SES as a treatment for dysphagia has increased the past few years, as described in the review study by Humbert et al. ${ }^{4}$. Despite the lack of scientific evidence of this treatment's efficacy for Parkinson's disease, dysphagic patients have been treated with SES in clinical practice. In that light, the present study describes the effects of SES of the submental region using different electrical current intensities as adjunct to traditional logopedic dysphagia treatment in dysphagic Parkinson patients.

\section{Materials and methods}

\section{Data collection}

\section{Participants}

For this study, patients with idiopathic Parkinson's disease and dysphagic complaints were recruited from diverse hospitals. The Hoehn and Yahr $(\mathrm{H}-\mathrm{Y})$ disability score was used to assess disease severity ${ }^{5}$. The oropharyngeal dysphagic complaints of the participants ranged from mild to severe. These included slow mastication and eating, oropharyngeal pooling, coughing while drinking or eating, choking on food, losing weight etc. All patients underwent a standardized clinical examination to ensure that the patient met the inclusion criteria. Patients were only accepted if they had followed an unaltered protocol of antiparkinsonian medication for at least 2 months and their disease was in a stable period. There were several exclusion criteria: presenting with any other neurological disease (besides Parkinson's); scoring below 23 on a Mini Mental State Examination (MMSE) ; being older than 80 years (presbyphagia); not being able to perform a swallow (aphagia); having had deep brain stimulation (DBS); exhibiting severe dyskinesia of head and neck (resulting in problems during the examinations); suffering from severe mental depression, head and neck cancer, or severe cardiopulmonary disease; having had speech therapy during the past six months; and having undergone surgery of the swallowing mechanism or the central nervous system. Written informed consent was obtained from all patients. The study protocol was approved by the medical ethical committee. 


\section{Randomization and study design}

Parkinson patients were assigned consecutively to one of the three treatment groups in the order in which they entered the study (quasi-random allocation). That is, the first patient was assigned to group 1, the second to group 2, the third to group 3 , and the fourth to group 1, etc. Patients in all three groups received daily one treatment session of 30 minutes for 15 days, but not during the weekend. All groups received traditional logopedic dysphagia treatment: for example, diverse airway protecting maneuvers; postural compensation maneuvers; bolus modification and oral intake of various foods; swallowing saliva; and oral motor exercises. Logopedic dysphagia treatment includes rehabilitative techniques to improve or restore the swallowing physiology and to facilitate dietary oral intake such as exercises to improve sensorimotor integration and muscle strength, and (postural) maneuvers. The selection was based on the dysphagic findings and the individual preference of the speech therapist. Apart from this treatment, the second and the third groups received SES of the submental region. Motor-level stimulation was performed in group 2, and sensory-level stimulation was performed in group 3. So logopedic swallowing therapy and eating were performed in conjunction with SES (at the same time). The treatment sessions and all examinations were performed during the "on" motor phase (within 90-120 minutes after the intake of antiparkinsonian medication $)^{7}$.

\section{Technique of surface electrical stimulation}

Eighty-five speech therapists with experience in traditional logopedic dysphagia treatment took part in the study.

The large number of therapists minimized the possibility of a therapist effect on group performance or treatment outcome. Each therapist recorded information in a patient journal (electrical current intensities, types of food, exercises or maneuvers). All therapists were invited to the university medical center to learn to use the surface electrical stimulator. The training was based on the manual of the manufacturer, the VitalStim certification course (http://www.vitalstim.com), and the study by Ludlow et al. ${ }^{8,9}$. The therapists performed supervised test trials with SES on patients and healthy subjects to ensure a standardized application of SES. They practiced the correct submental placement of the electrodes, the functioning of the SES unit, and the correct setting of the motor and sensory threshold of the electrical current. The therapists received a CD-ROM containing a demonstration video of the application of the electrical stimulator to maintain their level of experience at home. A commercially available electrical stimulator was used (VitalStim ${ }^{\circledR}$ Therapy; frequency $80 \mathrm{~Hz}$, pulse width $700 \mu \mathrm{s}$, Chattanooga Group, Chattanooga, TN, USA). Two skin electrodes (VitalStim ${ }^{\circledR}$, reference 59035), placed on the submental region and connected on each side of the midline of the neck, were selected as the electrode position on the basis of previous studies (Figure 5.1 ${ }^{8,10}$. The intensity of the electrical current ranged from 0 to 25 milliamperes $(\mathrm{mA})$. The maximum tolerated stimulation level resulting in maximum muscle contraction without spasm was applied as motor-level 
stimulation ${ }^{8,10}$. The sensory stimulation level was reached by raising the current intensity gradually until the first sensation of stimulation was felt by the patient. During each treatment session, the motor and the sensory thresholds were reconfirmed several times to correct for sensory adaptation and muscle fatigue.

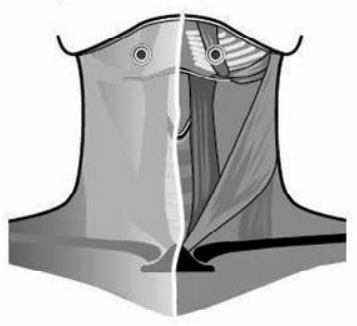

Figure 5.1 Electrode position (after cleaning, lifting, and shaving the skin): two electrodes placed horizontally above the hyoid bone on each side of the midline of the neck (submental region).

\section{Swallowing examination}

Data on the swallowing function was collected immediately before and after therapy by means of fiberoptic endoscopic evaluation of swallowing (FEES) and videofluoroscopy (VFS) in all patients. During the FEES examination, the patients were offered three trials of thin and three trials of thick liquid followed by three small bite-sized crackers. Each liquid trial contained $10 \mathrm{cc}$ of water or applesauce and was dyed with $5 \%$ methylene blue. The tip of the flexible fiberoptic endoscope Pentax FNL-10RP3 (Pentax Canada Inc., Mississauga, Ontario, Canada) was positioned just above the epiglottis in what is called the "high position" 11 . FEES images were obtained using an Alphatron Stroboview ACLS camera, Alphatron Lightsource, IVACX computerized video archiving system (Alphatron Medical Systems, Rotterdam, The Netherlands), and recorded on a DVD. Neither a nasal vasoconstrictor nor a topical anesthetic had been administered to the nasal mucosa. The VFS protocol consisted of three trials of $10 \mathrm{cc}$ low-density barium boluses ( $40 \% \mathrm{w} / \mathrm{v})$, three trials of $10 \mathrm{cc}$ thickened barium boluses (50 cc applesauce $+50 \mathrm{~g}$ barium powder), and three bite-sized crackers coated with barium paste. The field of the VFS image included the lips, the oral cavity, the cervical spine, and the proximal cervical esophagus (in lateral position; dental prosthesis in position). VFS images were obtained with a Philips Diagnost 97 system (Philips Medical Systems, Eindhoven, The Netherlands) and recorded on cassette at 25 frames per second using a mini-DV camera-recorder Panasonic AG-DVC30 (Matsushita Electric Industrial Co., Osaka, Japan). 


\section{Data analysis}

\section{Variables of interest}

Visuoperceptual ordinal variables were scored for each FEES and VFS swallow at varying speed (slow motion, normal, up to frame-by-frame speed) (Table 5.1). Before assessment of the swallowing acts, three experts received consensus training for these ordinal variables $^{12}$. The judges were blinded to the group, to the moment of measurement (pretherapy versus posttherapy), and to each other's ratings (independent rating) ${ }^{12}$. For FEES, the variables presented in Table 5.1 were assessed $^{12-16}$. The same variables were scored for VFS, supplemented with several more (Table 5.1) ${ }^{12-16}$. The trials of all subjects were scored in randomized order. To obtain the intrarater reliability, each rater performed repeated measurements (again blinded) of all visuoperceptual variables (FEES and VFS) during the second and third swallow of each bolus consistency for 10 randomly selected patients during pretherapy and posttherapy assessment ( $\mathrm{N}=120$ swallows per rater). This was done within a period of 2 weeks. Furthermore, raters were advised to limit the duration of the measurement sessions (max. 2 hours per session) to avoid fatigue and prevent instability of raters' characteristics.

\section{Statistical analysis}

Reliability analysis was performed using a weighted kappa $(\kappa)$ index of agreement (intrarater and interrater) for all visuoperceptual ordinal variables ${ }^{12,17}$. For reliable variables (i.e., Cohen's $k \geq 0.6$ ), further inferences were drawn based on the data of the rater with the highest intrarater reliability scores. Weighted $k$ values were computed and shown to be larger than the unweighted ones (most disagreements were thus of one category only).

Multilevel proportional odds models (POMs) were used to test for treatment effects over time, adjusting for the participant's sex and different bolus consistencies. For all POMs, the fixed effects were time (pretherapy versus posttherapy measurement moment), group (three treatment groups), and the effect of the bolus consistency (three different consistencies) on the ordinal outcome variables. Subjects were taken as a random factor. A random intercept was included to account for correlation among observations induced by repeated measurements on the same subject. For all models, interaction terms between treatment group and time (pretherapy versus postherapy measurement moment) were considered but then dropped due to the lack of statistical significance. Statistical analyses were performed using SAS statistical software, version 9.1.3 (SAS Institute Inc., Cary, NC, USA). A statistical model frequently used in clinical research to analyze ordinal outcome variables is $\mathrm{POM}^{18}$. Despite POM's widespread application, the proportionality of odds assumption is very stringent and needs to be checked to warrant validity of inference based on the model. Accordingly, this assumption was checked by performing separate binary logistic regressions. In the event of its violation, odds ratios (ORs) obtained via the logistic regressions would be reported instead. For parsimonious 
reasons, only one OR (of several binary logistic models) is presented, namely the one where the ordinal outcome was dichotomized into normal versus all other categories (pathological to different degrees).

Table 5.1 Definition of the visuoperceptual ordinal variables used to perform measurements in VFS and FEES.

\begin{tabular}{|c|c|c|c|c|}
\hline $\begin{array}{l}\text { Swallowing Assessment } \\
\text { Tool }\end{array}$ & $\begin{array}{l}\text { Visuoperceptual outcome } \\
\text { variable }\end{array}$ & Definition & Scale $^{a}$ & Reliability $^{\mathrm{b}}$ \\
\hline \multirow[t]{10}{*}{$\begin{array}{l}\text { Videofluoroscopy of } \\
\text { swallowing (VFS) }\end{array}$} & Preswallow anterior spill & $\begin{array}{l}\text { Preswallow loss of bolus from } \\
\text { the lips }\end{array}$ & $\begin{array}{l}\text { Five-point } \\
\text { scale }(0-4)\end{array}$ & $\begin{array}{l}\text { Not } \\
\text { applicable }\end{array}$ \\
\hline & Preswallow posterior spill ${ }^{c}$ & $\begin{array}{l}\text { Preswallow loss of bolus into } \\
\text { the pharynx }\end{array}$ & $\begin{array}{l}\text { Five-point } \\
\text { scale }(0-4)\end{array}$ & $\begin{array}{l}\text { Not } \\
\text { applicable }\end{array}$ \\
\hline & Lingual pumping & $\begin{array}{l}\text { Preswallow involuntary } \\
\text { repetitive tongue movements }\end{array}$ & $\begin{array}{l}\text { Five-point } \\
\text { scale }(0-4)\end{array}$ & Not reliable \\
\hline & Swallow hesitancy & Delayed onset oral transport & $\begin{array}{l}\text { Three-point } \\
\text { scale }(0-2)\end{array}$ & Not reliable \\
\hline & Piecemeal deglutition & $\begin{array}{l}\text { Sequential swallowing of the } \\
\text { same bolus }\end{array}$ & $\begin{array}{l}\text { Five-point } \\
\text { scale (0-4) }\end{array}$ & Reliable \\
\hline & $\begin{array}{l}\text { Delayed initiation pharyngeal } \\
\text { reflex }\end{array}$ & $\begin{array}{l}\text { Delayed onset pharyngeal } \\
\text { triggering }\end{array}$ & $\begin{array}{l}\text { Three-point } \\
\text { scale }(0-2)\end{array}$ & Not reliable \\
\hline & Postswallow oral residue & $\begin{array}{l}\text { Pooling in the oral cavity after } \\
\text { the swallow }\end{array}$ & $\begin{array}{l}\text { Five-point } \\
\text { scale }(0-4)\end{array}$ & Not reliable \\
\hline & $\begin{array}{l}\text { Postswallow vallecular } \\
\text { pooling }\end{array}$ & $\begin{array}{l}\text { Pooling in the valleculae after } \\
\text { the swallow }\end{array}$ & $\begin{array}{l}\text { Three-point } \\
\text { scale }(0-2)\end{array}$ & Reliable \\
\hline & $\begin{array}{l}\text { Postswallow pyriform sinus } \\
\text { pooling }\end{array}$ & $\begin{array}{l}\text { Pooling in the pyriform } \\
\text { sinuses after the swallow }\end{array}$ & $\begin{array}{l}\text { Three-point } \\
\text { scale (0-2) }\end{array}$ & Reliable \\
\hline & $\begin{array}{l}\text { Penetration-aspiration scale } \\
\text { of Rosenbek et al. }{ }^{d}\end{array}$ & Penetration and/or aspiration & $\begin{array}{l}\text { Eight-point } \\
\text { scale }(1-8)\end{array}$ & Reliable \\
\hline \multirow{6}{*}{$\begin{array}{l}\text { Fiberoptic endoscopic } \\
\text { evaluation of } \\
\text { swallowing (FEES) }\end{array}$} & Preswallow posterior spill ${ }^{c}$ & $\begin{array}{l}\text { Preswallow loss of bolus into } \\
\text { the pharynx }\end{array}$ & $\begin{array}{l}\text { Five-point } \\
\text { scale (0-4) }\end{array}$ & Reliable \\
\hline & Piecemeal deglutition & $\begin{array}{l}\text { Sequential swallowing of the } \\
\text { same bolus }\end{array}$ & $\begin{array}{l}\text { Five-point } \\
\text { scale (0-4) }\end{array}$ & Reliable \\
\hline & $\begin{array}{l}\text { Delayed initiation pharyngeal } \\
\text { reflex }\end{array}$ & $\begin{array}{l}\text { Delayed onset pharyngeal } \\
\text { triggering }\end{array}$ & $\begin{array}{l}\text { Three-point } \\
\text { scale }(0-2)\end{array}$ & Reliable \\
\hline & $\begin{array}{l}\text { Postswallow vallecular } \\
\text { pooling }\end{array}$ & $\begin{array}{l}\text { Pooling in the valleculae after } \\
\text { the swallow }\end{array}$ & $\begin{array}{l}\text { Three-point } \\
\text { scale }(0-2)\end{array}$ & Reliable \\
\hline & $\begin{array}{l}\text { Postswallow pyriform sinus } \\
\text { pooling }\end{array}$ & $\begin{array}{l}\text { Pooling in the pyriform } \\
\text { sinuses after the swallow }\end{array}$ & $\begin{array}{l}\text { Three-point } \\
\text { scale }(0-2)\end{array}$ & Not reliable \\
\hline & $\begin{array}{l}\text { Penetration-aspiration scale } \\
\text { of Rosenbek et al. }\end{array}$ & Penetration and/or aspiration & $\begin{array}{l}\text { Eight-point } \\
\text { scale }(1-8)\end{array}$ & Not reliable \\
\hline
\end{tabular}

${ }^{a}$ Lower scores refer to normal functioning whereas higher scores refer to more severe disability . ${ }^{b}$ Reliable: Weighted $k \geq 0.60$. ${ }^{\text {P }}$ Preswallow posterior spill was not scored for bite-sized crackers. ${ }^{\mathrm{d}}$ Rosenbek et al. ${ }^{14}$.

\section{Results}

\section{Characteristics of participants}

The study included 109 mentally competent patients with a diagnosis of idiopathic Parkinson's disease and oropharyngeal dysphagic complaints (Figure 5.2). During the period of intervention, 19 subjects were defined as dropouts for various methodological 
reasons (change of antiparkinsonian medication during $\mathrm{SES}, \mathrm{N}=15$; dental surgery during $\mathrm{SES}, \mathrm{N}=2$; other reasons, $\mathrm{N}=2$ ). The excluded subjects did not experience adverse effects from therapy. Finally, each treatment group contained 30 patients (total $\mathrm{N}=90 ; 24$ females and 66 males).

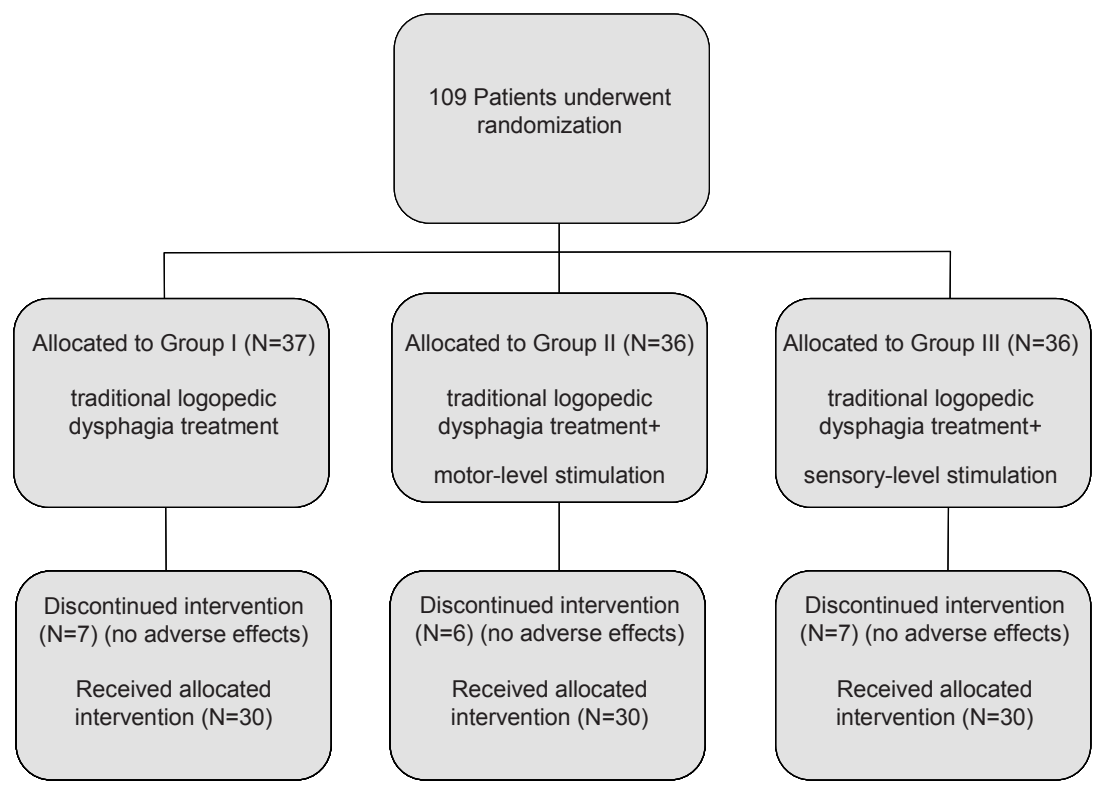

Figure 5.2 Flow diagram of the three treatment groups. The diagram includes detailed information on the number of participants per group.

Before treatment, no important differences were found in baseline characteristics among the three treatment groups. The median age of all patients was 68 years. The H-Y scale ranged from I to $\mathrm{V}$ (median=II). ${ }^{5}$ The duration of the Parkinson's disease was at least five years. The median current intensity for group 2 was $10.5 \mathrm{~mA}\left(25^{\text {th }}, 75^{\text {th }}\right.$ percentile: $7.25 ; 14.0)$ versus $3.25 \mathrm{~mA}\left(25^{\text {th }}, 75^{\text {th }}\right.$ percentile: $\left.3.0 ; 4.38\right)$ for group 3.

\section{Reliability analysis}

VFS visuoperceptual (ordinal) variables - specifically, preswallow anterior and preswallow posterior spill, and swallow hesitancy - mainly scored zero points, indicating normal without any disturbances. It was decided to exclude these VFS variables because of insufficient relevance to the present patient group ${ }^{12,17}$. For several variables in FEES and VFS, the intrarater and interrater reliability was sufficient (Table 5.1). 


\section{Results of the Proportional Odds Model (POM)}

The three variables - time (therapy effect), representing differences in odds for being in healthier categories between pretherapy and posttherapy measurements for FEES and VFS; group, representing effects of different therapies; and bolus consistency - made up the fixed effects of all POMs, irrespective of their statistical significance. Interaction terms between treatment group and time were also added to the model but were always dropped due to lack of statistical significance. Adjusted ORs for those FEES and VFS variables showing significant improvement after the traditional logopedic dysphagia treatment are presented in Figure 5.3 and Table 5.2. For the variables in italics in Figure 5.3 , the POM assumption was violated. ORs were thus based on the binary logistic models (normal versus all other categories). In general, the ORs express how much the odds that a patient will be classified in healthier (lower) outcome categories have changed over time, for example, due to traditional logopedic dysphagia treatment. (Note that the OR is always to be interpreted with respect to the reference category, the posttherapy measurement). Differences among the groups' odds, representing an additional SES treatment effect, could not be observed for any of the visuoperceptual FEES and VFS variables. For example, one VFS variable, piecemeal deglutition, improved after therapy for all of the dysphagic Parkinson patients, irrespective of their group allocation. The OR of 0.73 pretherapy with respect to posttherapy indicates that the chance of being classified in healthier categories of the ordered response was smaller at the baseline measurement than after the therapy.

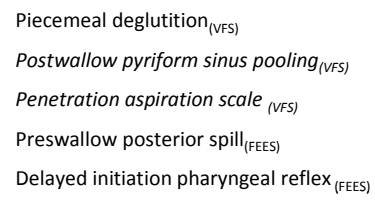

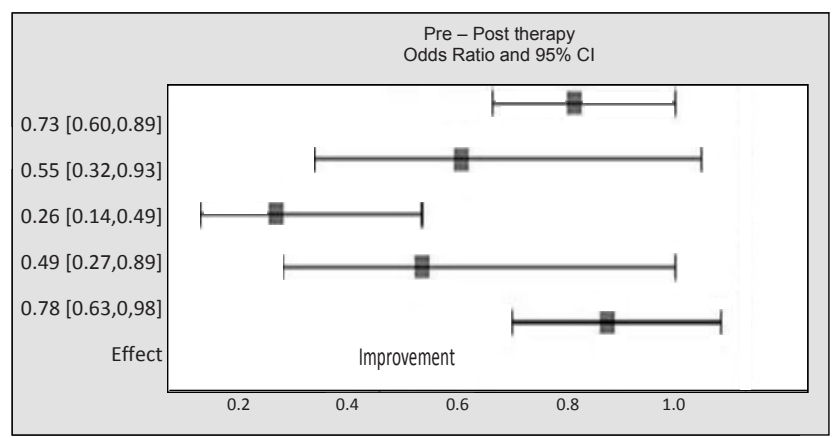

Figure 5.3 Forest plot displaying ORs and corresponding 95\% Cl for patients to be classified in healthier categories for those FEES and VFS outcome variables showing statistically significant improvement after traditional logopedic dysphagia treatment. ORs were estimated by either mixed POM or mixed binary logistic regression models (outcome variables in italics), adjusted for patients' sex and bolus consistencies. All ORs, computed in the direction of pretherapy with respect to posttherapy moments of measurement, are smaller than " 1 ", indicating that the odds of a patient to be classified in healthier (for POMs) or normal categories (for binary logistic models) are significantly smaller before therapy than afterwards. $\mathrm{Cl}=$ confidence interval; FEES=fiberoptic endoscopic evaluation of swallowing; OR=odds ratio; POM=proportional odds model; VFS=videofluoroscopy of swallowing. 
A similar time effect was observed for the FEES variables of preswallow posterior spill (liquid boluses) and delayed initiation pharyngeal reflex; both showed significant improvement after any therapy. In addition to the pretherapy versus posttherapy effect, several FEES and VFS variables were shown to change significantly, depending on the consistency of the applied boluses. This effect, which was not related to SES, was observed regardless of the moment of measurement (pretherapy versus posttherapy) and irrespective of the group (Table 5.2). Such findings - for example, less vallecular pooling during VFS for thin liquid compared to crackers - are not surprising as the effects of bolus consistency on swallowing in patients with Parkinson's disease have been described in previous studies ${ }^{19}$.

Table 5.2 Estimated odds ratios (ORs) according to the fitted proportional odds model for the entire study population using reliable visuoperceptual ordinal variables $(\mathrm{N}=90)$.

\begin{tabular}{|c|c|c|c|c|c|c|c|}
\hline \multirow[t]{2}{*}{$\begin{array}{l}\text { Swallowing } \\
\text { Assessment Tool }\end{array}$} & \multirow[t]{2}{*}{$\begin{array}{l}\text { Reliable } \\
\text { outcome } \\
\text { variable }\end{array}$} & \multicolumn{2}{|c|}{$\begin{array}{l}\text { Moment of } \\
\text { measurement } \\
\text { effect (pretherapy } \\
\text { vs. posttherapy) }\end{array}$} & \multirow[t]{2}{*}{ Explanation } & \multicolumn{2}{|c|}{$\begin{array}{l}\text { Bolus consistency } \\
\text { effect }^{c}\end{array}$} & \multirow[t]{2}{*}{ Explanation } \\
\hline & & OR & {$[95 \% \mathrm{Cl}]^{\mathrm{b}}$} & & $\mathrm{OR}$ & {$[95 \% \mathrm{Cl}]$} & \\
\hline \multirow[t]{2}{*}{$\begin{array}{l}\text { Videofluoroscopy } \\
\text { of swallowing } \\
\text { (VFS) }\end{array}$} & $\begin{array}{l}\text { Piecemeal } \\
\text { deglutition }\end{array}$ & 0.73 & {$[0.60,0.89]$} & $\begin{array}{l}\text { Improvement } \\
\text { after } \\
\text { treatment }\end{array}$ & 7.043 & {$[4.54,10.90]$} & $\begin{array}{l}\text { Less piecemeal deglutition for } \\
\text { thin liquid compared to } \\
\text { crackers }\end{array}$ \\
\hline & $\begin{array}{l}\text { Postswallow } \\
\text { vallecular } \\
\text { pooling }\end{array}$ & Not si & snificant & - & 11.30 & {$[6.47,19.71]$} & $\begin{array}{l}\text { Less pooling for thin liquid } \\
\text { compared to crackers }\end{array}$ \\
\hline \multirow{4}{*}{$\begin{array}{l}\text { Fiberoptic } \\
\text { endoscopic } \\
\text { evaluation of } \\
\text { swallowing (FEES) }\end{array}$} & $\begin{array}{l}\text { Preswallow } \\
\text { posterior spill } \\
\text { (liquid boluses) }^{d}\end{array}$ & 0.49 & {$[0.27,0.89]$} & $\begin{array}{l}\text { Improvement } \\
\text { after } \\
\text { treatment }\end{array}$ & 0.30 & {$[0.10,0.89]$} & $\begin{array}{l}\text { Less spill for thick liquid } \\
\text { compared to thin liquid }\end{array}$ \\
\hline & $\begin{array}{l}\text { Piecemeal } \\
\text { deglutition }\end{array}$ & \multicolumn{2}{|c|}{ Not significant } & - & 2.36 & {$[1.56,3.56]$} & $\begin{array}{l}\text { Less piecemeal deglutition for } \\
\text { thin liquid compared to } \\
\text { crackers }\end{array}$ \\
\hline & $\begin{array}{l}\text { Delayed } \\
\text { initiation } \\
\text { pharyngeal } \\
\text { reflex }\end{array}$ & 0.78 & {$[0.63,0.96]$} & $\begin{array}{l}\text { Improvement } \\
\text { after } \\
\text { treatment }\end{array}$ & 0.35 & {$[0.22,0.54]$} & $\begin{array}{l}\text { Less delayed initiation } \\
\text { pharyngeal reflex for crackers } \\
\text { compared to thin liquid }\end{array}$ \\
\hline & $\begin{array}{l}\text { Postswallow } \\
\text { vallecular } \\
\text { pooling }\end{array}$ & \multicolumn{2}{|c|}{ Not significant } & - & 2.67 & {$[1.65,4.33]$} & $\begin{array}{l}\text { Less postswallow vallecular } \\
\text { pooling for thin liquid } \\
\text { compared to crackers }\end{array}$ \\
\hline
\end{tabular}

For all models, the groups, moments of measurement (pretherapy versus posttherapy), and bolus consistencies were added as explanatory variables. All proportional odds models are modeling the probability of the patients' outcome falling into lower categories of the ordered response profiles. The $95 \%$ confidence interval is presented. ${ }^{a}$ Direction of the effect, i.e. for moment of measurement (pretherapy versus posttherapy). The corresponding OR was computed with the posttherapy value taken as a reference. ${ }^{b} 95 \% \mathrm{Cl}$ : the $95 \%$ confidence interval. ${ }^{c}$ Direction of the effect, i.e. for bolus consistency ( $3 \times$ thin liquid, $3 \times$ thick liquid, $3 \times$ bite-sized cracker). The corresponding OR was computed with solid consistency bite-sized cracker, taken as a reference. ${ }^{\mathrm{d}}$ Preswallow posterior spill was not scored for bite-sized crackers. 


\section{Discussion}

The treatment of oropharyngeal dysphagia in Parkinson patients has become an important topic because of the growing population of these patients and the increasing concern about quality of life for dysphagic patients ${ }^{1}$. Helping these patients enjoy food would enhance their quality of life ${ }^{20}$. Indeed, an important reason to conduct the present study was to justify the application of SES in dysphagic Parkinson patients in daily practice. Ultimately, the study might further the development of a treatment to decrease the risks and the medical costs of dysphagia.

The pathophysiology of dysphagia in Parkinson's disease is complex. It depends on the coordination of factors in both the peripheral and the central nervous system. Oropharyngeal dysphagia can be caused by uncoordinated or disrupted signals along dopaminergic and nondopaminergic neural pathways. On the grounds of clinical experience and the literature, it was assumed that sensory-level and motor-level SES of the submental region could alter the swallow physiology in Parkinson's' disease by modulating these complex nervous system factors ${ }^{17}$. SES did not elicit significant group differences in the present study. There may be a multitude of reasons for this lack of statistical significance of SES. The fixed stimulation variables (frequency $=80 \mathrm{~Hz}$, pulse width $=700 \mu \mathrm{s}$, current intensity $=0-25 \mathrm{~mA}$ ) of the VitalStim electrical stimulator may not have been appropriate to induce any therapy effect during 15 days of SES in dysphagic Parkinson patients. According to other authors, oropharyngeal excitability depends on the stimulation parameters applied ${ }^{21-23}$. It is expected that different stimulation variables, or different values of the stimulation variables for SES, may cause therapy effects in dysphagic Parkinson patients. Furthermore, oropharyngeal dysphagia in the presence of Parkinson's disease can be due to dysfunction of central and peripheral nervous system pathways of swallowing rather than to muscle weakness or peripheral sensory dysfunction, for which the applied electrical stimulation device was mainly designed ${ }^{9}$. Another reason for the absence of therapy effects of SES may be that snap skin electrodes might not be a precisely targeted method of electrical stimulation. It is quite possible that the technique stimulated (antagonistic) muscles, or other tissues that are not involved in the swallowing physiology. Also, the choice of the stimulation site, the submental region, may not be optimal to modulate swallowing or to influence the central and peripheral nervous systems in Parkinson's disease. Perhaps other anatomical sites are more susceptible to the reception and transduction of electrical current ${ }^{24}$. However, more research is needed in this field. Another limitation could be that visuoperceptual ordinal variables were scored for each FEES and VFS swallow. The measurement of continuous spatial or temporal variables may have leaded to different results. However, the very large number of measurements in the present patient population (more than 97.000 measurements) resulted in a very labor-intensive project for the team of independent raters. Furthermore, the length of treatment in the present study can be a reason for the less-significant effects of SES. A period longer than 15 days and a session longer than 30 minutes may cause therapeutic effects in the upper aerodigestive tract or neural 
pathways of dysphagic Parkinson patients. However, after 30 minutes of treatment, several patients showed signs of fatigue. Finally, the duration of the "on" motor phase was limited in some patients. A longer session would entail a risk of treating them during the "off" motor phase. The result would be a less-effective treatment outcome due to decreased swallowing function or increased dyskinesia during this phase ${ }^{25,26}$. Fatigue or treatment during the "off" motor phase can lead to instability of the patients' characteristics, resulting in bias.

To avoid methodological shortcomings, several precautions were taken. For example, all pretherapy and posttherapy measurements were performed in the same hospital by the same multidisciplinary team in order to guarantee standardized data collection. The median score of II on the H-Y scale for the present study population could also have led to finding therapy effects that are less significant. Still, the study population was a reflection of the theoretical population of dysphagic Parkinson patients who consult speech therapists for dysphagia treatment. Several patients with severe Parkinson's disease $(\mathrm{H}-\mathrm{Y}$ score IV-V) were excluded because of cognitive impairment (MMSE<23) or because of their extremely weak condition.

\section{Conclusion}

After 15 days of treatment, no statistically significant differences in FEES and VFS outcome variables were found between any of the three treatment groups (motor-level or sensorylevel SES of the submental region as adjunct to traditional logopedic dysphagia treatment versus traditional logopedic dysphagia treatment solely). These preliminary findings are open to interpretation. At least under the present study protocol, they suggest that SES treatment has no added value in the treatment of dysphagic Parkinson patients. Alternatively, the lack of improvement after a short period of SES highlights the need to explore and understand questions like the exact mechanism of SES and its effects on the neural pathways involved in swallowing. Further research should focus on values of stimulation parameters; different stimulation sites; simultaneous EMG feedback during SES; and choices about the length of a treatment period, the number of sessions, and their duration. 


\section{References}

1. Jankovic J, Tolosa E. Parkinson's disease \& movement disorders fifth edition. Philadelphia: Lippincott Williams \& Wilkins, Wolters Kluwer Business; 2007.

2. Hunter PC, Crameri J, Austin S, Woodward MC, Hughes J. Response of parkinsonian swallowing dysfunction to dopaminergic stimulation. J Neurol Neurosurg Psychiatry. 1997; 63:579-583.

3. Baijens LWJ, Speyer R. Effects of Therapy for Dysphagia in Parkinson's Disease. Systematic Review. Dysphagia. 2009;24:91-102.

4. Humbert IA, Michou E, MacRae PR, Crujido L. Electrical stimulation and swallowing: how much do we know? Semin Speech Lang. 2012;33:203-216.

5. Hoehn NM, Yahr MD. Parkinsonism: onset, progression and mortality. Neurology. 1967;17:427-442.

6. Folstein MF, Folstein SE, McHugh PR. "Mini-mental state". A practical method for grading the cognitive state of patients for the clinician. J Psychiatr Res. 1975;12:189-198.

7. Wajsbort J. The "off-on" phenomenon during treatment of Parkinson's disease with Levodopa. J Neurol. 1997;215:59-66.

8. Ludlow CL, Humbert I, Saxon K, Poletto C, Sonies B, Crujido L. Effects of surface electrical stimulation both at rest and during swallowing in chronic pharyngeal dysphagia. Dysphagia. 2007;22:1-10.

9. Freed M, Wijting Y. VitalStim Certification Program. Training manual for patient assessment and treatment using VitalStim electrical stimulation. Hixson TN: Chattanooga Group; 2003.

10. Humbert I, Poletto C, Saxon K, et al. The effect of surface electrical stimulation on hyo-laryngeal movement in normal individuals at rest and during swallowing. J Appl Physiol. 2006;101:1657-1663.

11. Langmore SE, Aviv JE. Endoscopic evaluation and treatment of swallowing disorders. New York: Thieme; 2001.

12. Baijens L, Speyer R, Lima Passos V, Pilz W, Roodenburg N, Clavé P. Swallowing in Parkinson patients versus healthy controls: Reliability of measurements in videofluoroscopy. Gastroenterol Res Pract. 2011;2011: 380682

13. Ali GN, Wallace KL, Schwartz R, Decarle DJ, Zagami AS, Cook IJ. Mechanisms of oral-pharyngeal dysphagia in patients with Parkinson's disease. Gastroenterology. 1996;110: 383-392.

14. Rosenbek JC, Robbins JA, Roecker EB, Coyle JL, Wood JL. A penetration-aspiration scale. Dysphagia. 1996;11: 93-98.

15. Robbins JA, Logemann J, Kirshner H. Swallowing and speech production in Parkinson's disease. Ann Neurol. 1986;19:283-287.

16. Nagaya M, Teruhiko K, Yamada T, Igata A. Videofluorographic study of swallowing in Parkinson's disease. Dysphagia. 1998;13: 95-100.

17. Baijens LWJ, Speyer R, Lima Passos V, Pilz W, Roodenburg N, Clavé P. The effect of surface electrical stimulation on swallowing in dysphagic Parkinson patients. Dysphagia. 2012 Jan 12. Epub ahead of print.

18. Bender R, Grouven U. Using Binary Logistic Regression Models for Ordinal Data with Non-proportional Odds. J Clin Epidemiol. 1998; 51: 809-816.

19. Logemann JA, Gensler G, Robbins J, et al. A randomized study of three interventions for aspiration of thin liquids in patients with dementia or Parkinson's disease. J Speech Lang Hear Res. 2008;51:173-183.

20. Heijnen BJ, Speyer R, Baijens LWJ, Bogaardt HCA. Neuromuscular electrical stimulation versus traditional therapy in patients with Parkinson's disease and oropharyngeal dysphagia: Effects on quality of life. Dysphagia. 2012;27:336-345.

21. Fraser C, Rothwell J, Power M, Hobson A, Thompson D, Hamdy S. Differential changes in human pharyngoesophageal motor excitability induced by swallowing, pharyngeal stimulation, and anesthesia. Am J Physiol Gastrointest Liver Physiol. 2003;285:G137-144.

22. Gallas S, Marie JP, Leroi AM, Verin E. Sensory transcutaneous electrical stimulation improves post-stroke dysphagic patients. Dysphagia. 2010;25:291-297.

23. Furuta T, Takemura M, Tsujita J, Oku Y. Interferential electric stimulation applied to the neck increases swallowing frequency. Dysphagia. 2012;27:94-100.

24. Yamamura K, Kitagawa J, Kurose M, et al. Neural mechanisms of swallowing and effects of taste and other stimuli on swallow initiation. Biol Pharm Bull. 2010;33:1786-1790.

25. Tison F, Wiart L, Guatterie M, et al. Effects of central dopaminergic stimulation by apomorphine on swallowing disorders in Parkinson's disease. Mov Disord. 1996;11:729-732. 
Chapter 5

26. Fuh JL, Lee RC, Wang SJ, et al. Swallowing difficulty in Parkinson's disease. Clin Neurol Neurosurg. 1997;99:106-112. 


\section{Chapter 6}

FEES protocol derived estimates of sensitivity to aspiration in dysphagic patients: the number of swallow trials versus aspiration risk

Laura W.J. Baijens

Renée Speyer

Walmari Pilz

Nel Roodenburg

Submitted
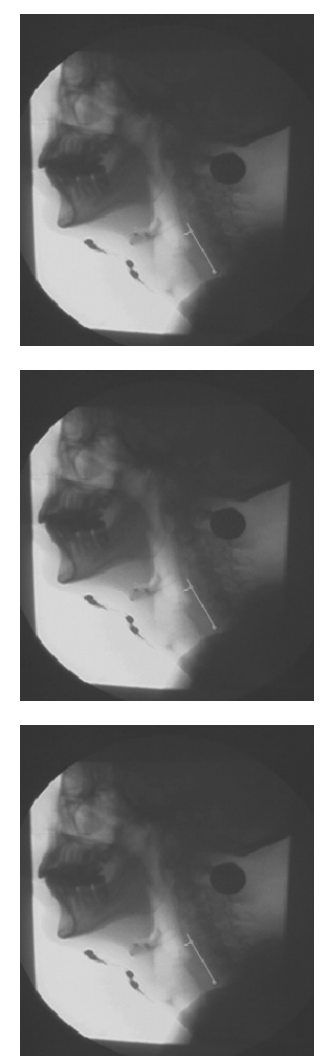


\section{Abstract}

The objective of this prospective study was to determine the FEES (fiberoptic endoscopic evaluation of swallowing) protocol derived estimates of sensitivity $\left(\mathrm{Se}^{\prime}\right)$ to detection of aspiration in dysphagic patients. The study estimated the probability of aspiration as a function of the number of swallow trials in dysphagic patients using FEES. The derived sensitivity was calculated based on presence or absence of aspiration in a ten-swallow trial protocol as arbitrary 'gold standard'. Eighty-four persons were included, comprising two patient populations with oropharyngeal dysphagia. Dysphagia in one group was due to head and neck cancer and possible oncological treatment effects on swallowing; in the other it was a result of neurological disease. All patients underwent a standardized FEES examination using ten swallows of thin liquid followed by ten swallows of thick liquid, all in boluses of $10 \mathrm{cc}$ each. FEES recordings were rated for aspiration by an expert panel blinded to patients' identity and clinical history. Descriptive statistics, Kaplan-Meier survival analysis techniques, and Log Rank/Mantel-Cox tests were used. In both patient populations the aspiration risk was underestimated when using a limited number (three or four) of swallow trials. The oncology and neurology patients differed significantly in the number of swallow trials required to determine aspiration for thin liquids (median values 2 and 7 respectively, $P=0.006$ ). FEES protocols using a limited number of swallow trials can underestimate the aspiration risk in both oncological and neurological patients suffering from oropharyngeal dysphagia, especially when using boluses with a thin liquid consistency. 


\section{Introduction}

Aspiration, defined as the passage of bolus below the level of the vocal folds, is common in patients with oropharyngeal dysphagia ${ }^{1,2,3}$. Severe dysphagia can result in aspiration, aspiration pneumonia, and sudden death, and the costs associated with dysphagiainduced comorbidity are high ${ }^{2}$. Fiberoptic endoscopic evaluation of swallowing (FEES) offers the dysphagia professional a reliable tool in case of penetration or aspiration ${ }^{4}$. FEES is well tolerated, easily repeatable, and can be performed at the bedside ${ }^{5}$. However, various protocols exist, and there is no consensus on the number of swallow trials, bolus consistencies, and bolus volumes to include in a FEES. The present prospective study was designed to estimate the probability of aspiration as a function of the number of swallow trials. It applies a standardized protocol calling for ten consecutive swallow trials of $10 \mathrm{cc}$ each in two different consistencies (first ten boluses of thin liquid, then ten boluses of thick liquid) administered to oncological and neurological patients suffering from oropharyngeal dysphagia.

\section{Materials and methods}

\section{Participants}

Patients with oropharyngeal dysphagia were divided into two diagnostic groups. In the one, dysphagia was due to head and neck cancer and possible oncological treatment effects on swallowing; in the other, dysphagia was accompanied by a neurological disease. The patients were consecutively enrolled in the present prospective study while visiting the outpatient clinic of the Maastricht University Medical Center for their dysphagic complaints. Their data were collected as part of the regular healthcare program for oropharyngeal dysphagia ${ }^{6}$. All patients reported subjective clinical complaints of oropharyngeal dysphagia ranging from mild to severe. These included, among others, slow eating due to prolonged transit times, oral or pharyngeal passage disorder, coughing while drinking, choking on foods, and aspiration pneumonia. All patients were able to perform a swallow on command. The oncological and neurological etiologies were heterogeneous. The following exclusion criteria were applied: a Mini Mental State Examination (MMSE) score below $23^{7}$; concurrent head and neck cancer and a neurological disease (or neurosurgical brain intervention); a stroke less than three months ago; head and neck oncological treatment less than three months ago; surgery of the head and neck swallowing region in patients with neurological disease; extreme fatigue or weakness; an unstable period of a neurological disease; not having had the same medication regimen for the past six weeks in neurological patients (i.e., with Parkinson's disease); and having undergone total laryngectomy. 


\section{Ethical considerations}

The study was carried out using standardized and anonymized data from the regular healthcare program for oropharyngeal dysphagia at the outpatient clinic $^{6}$. Informed consent was obtained from all patients as this is part of the regular healthcare program for oropharyngeal dysphagia. The study protocol was approved by the medical ethical committee.

\section{Swallowing assessment}

Before the FEES, the severity of oropharyngeal dysphagia was assessed using the Functional Oral Intake Scale (FOIS) ${ }^{8}$ and a clinical observation of oral intake by a speech and language pathologist. Furthermore, a detailed clinical examination was performed by an experienced laryngologist to ensure correct inclusion. The range of scores on the FOIS is one to seven, indicating nothing by mouth (1) to total oral diet with no restrictions ${ }^{8}$. After these preliminaries, all subjects underwent a standardized FEES protocol. During the FEES, the patients were offered ten trials of thin liquid followed by ten trials of thick consistency. Each trial contained $10 \mathrm{cc}$ of water (thin liquid) or applesauce (thick liquid) and was dyed with five percent methylene blue $(10 \mathrm{mg} / \mathrm{ml})$. The order of presenting boluses of different consistencies was based on the international literature and the range of standardized protocols usually followed in clinical practice ${ }^{9,10}$. The tip of the flexible fiberoptic endoscope Pentax FNL-10RP3 (Pentax Canada Inc., Mississauga, Ontario, Canada) was positioned just above the epiglottis in the 'high position' so as not to compromise the closure of the laryngeal vestibule ${ }^{5}$. FEES images were obtained using an Alphatron Stroboview ACLS camera, Alphatron Lightsource, IVACX computerized video archiving system (Alphatron Medical Systems, Rotterdam, The Netherlands) and recorded on a DVD. Neither a nasal vasoconstrictor nor a topical anesthetic was administered to the nasal mucosa. The subjects had to swallow the bolus after it had been accurately delivered by syringe in the oral cavity. They were wearing their dental prosthesis (if present). In the event of aspiration during a trial, the examination using this consistency was ended.

\section{Outcome measurements}

Swallows were analyzed using the visuoperceptual (dichotomous) variable of aspiration (present or absent). Any material entering the airway below the true vocal folds was defined as aspiration ${ }^{3}$. Prior to the actual rating procedure, a panel was formed of two experienced raters who were trained to interpret and score the dichotomous scale. After that experimental run, they carried out the procedure on the raw data, identifying and rating all swallow trials on the basis of consensus. The panel was blinded to the diagnostic group and to the identity of the patients. Each rater had more than eight years experience in judging FEES videos in daily practice and during previous scientific studies ${ }^{6,9}$. Indeed, their intrarater and interrater reliability for scoring aspiration proved reliable in previous studies (Weighted Kappa $\geq 0.60$ ). ${ }^{9}$ These experts scored the blocks of FEES videos (ten 
swallow acts per consistency) in randomized order. Each swallow could be assessed at varying speed, ranging from normal to slow motion up to frame-by-frame, as many times as necessary. The duration of the panel sessions was limited by the raters themselves (max. two hours per session).

\section{Statistical methods}

All statistical analyses were performed using IBM SPSS Statistics for Windows, Version 20.0 (IBM Corp., Armonk, New York, USA). Descriptive statistics on the number of swallow trials were assembled for each consistency separately. Presence or absence of aspiration was determined arbitrarily at the tenth swallow trial (if applicable); If no aspiration had occurred during any of the ten consecutive trials (per consistency), the patient was declared a non-aspirator. If aspiration had occurred, that patient was considered an aspirator. FEES is considered to be a gold standard in the assessment of oropharyngeal dysphagia $^{11}$. Sensitivity testing refers to how the final outcome of an analysis may change as a function of varying one or more of the input parameters (such as number of swallow trials) in a prescribed manner ${ }^{12}$. In mathematics, the prime symbol ( $\left.{ }^{\prime}\right)$ is generally used to generate a variable that is related or similar to an original variable or concept but not identical (notation, for example, variable $x^{\prime}$ versus variable $\mathrm{x}$ ). To distinguish the derived estimates of sensitivity as determined in this study from sensitivity calculation using an external gold standard or different assessment tool, the variable $\mathrm{Se}^{\prime}$ was introduced. Derived estimates of sensitivity ( $\mathrm{Se}^{\prime}$ ) for aspiration were determined for the total group as a function of the number of swallow trials used in a FEES protocol. Crosstabs calculations based on a dichotomized classification (aspirator versus non-aspirator) using an arbitrarily 'gold standard' (tenth swallow trial), provided the derived sensitivity data for both consistencies separately. Kaplan-Meier survival analysis techniques were used to visualize the occurrence of aspiration as a function of the number of swallow trials per group and consistency. The resulting differences in occurrence between the diagnostic groups were tested for significance by means of the Log Rank/Mantel-Cox test for both consistencies.

\section{Results}

\section{Participants}

The study included eighty-four mentally competent and physically stable patients (18 9 , $66 \hat{)}$ ) with oropharyngeal dysphagia in two etiological groups ( $N=50$ neurology, $N=34$ oncology). The mean age of the oncological patients was 67 years, that of the neurological patients 60 . The median of the FOIS score was 4 in the oncological patients and 5 in the neurological patients (Table 6.1). 
Table 6.1 Patient characteristics.

\begin{tabular}{lcccc}
\hline Patient population & $\mathrm{N}$ & Gender & Mean age (Range) & Median FOIS (Range) \\
\hline Total population & 84 & $18+, 66 \hat{0}$ & $63(21-85)$ & $5(1-7)$ \\
Neurological subjects & 50 & $13+, 37 \hat{0}$ & $60(21-85)$ & $5(2-7)$ \\
Oncological subjects & 34 & $5+, 290$ & $67(30-83)$ & $4(1-7)$ \\
\hline
\end{tabular}

FOIS: functional oral intake scale

\section{Aspiration risk as a function of the number of swallow trials in FEES}

Table 6.2 presents descriptive data on aspiration risk for thin and thick consistencies as a function of the number of trials used in FEES. The total number of patients (column 3) per trial decreases as the number of trials rises because the examination was ended when patients showed aspiration. The table gives the number of patients with and without aspiration (column 4). Fewer patients received a bolus with thick liquid than one of thin liquid (66 versus 84 subjects) because the examination of several patients was stopped after the first ten boluses for various reasons: very severe dysphagia with massive aspiration during administration of the thin consistency; exam was too tiring; unpleasant taste of the boluses, etc. Very few showed massive aspiration when offered the first consistency. Those who did were instructed to cough in order to eject the inhaled bolus, and they underwent a clinical follow-up regimen after the FEES. None of these patients developed pneumonia, and no other adverse effects were observed.

Table 6.2 Descriptive statistics of aspiration risk as a function of number of swallow trials in FEES.

\begin{tabular}{|c|c|c|c|c|c|}
\hline $\begin{array}{l}\text { Bolus } \\
\text { consistency }\end{array}$ & $\begin{array}{c}\text { Number of } \\
\text { swallow trial }\end{array}$ & $\begin{array}{c}\text { Total number of } \\
\text { subjects per swallow } \\
\text { trial*: } \mathrm{N}_{\text {total }}\end{array}$ & $\begin{array}{l}\text { Data per swallow trial: } \\
\mathrm{N}_{\text {aspiration }}(\%) ; \mathrm{N}_{\text {normal }}(\%)\end{array}$ & $\begin{array}{c}\text { Cumulative data: } \\
\mathrm{N}_{\text {aspiration }}(\%) ; \mathrm{N}_{\text {normal }}(\%)\end{array}$ & $\begin{array}{c}\text { Se' of aspiration } \\
\text { (\%) per swallow } \\
\text { trial** }\end{array}$ \\
\hline \multirow{10}{*}{$\begin{array}{l}\text { Thin liquid } \\
\text { (10 cc) }\end{array}$} & 1 & 84 & 25(29.8); 59(70.3) & 25(29.8); 59(70.3) & 43.9 \\
\hline & 2 & 59 & $9(15.3) ; 50(84.8)$ & $34(40.5) ; 50(59.5)$ & 59.6 \\
\hline & 3 & 50 & $5(10.0) ; 45(90.0)$ & $39(46.4) ; 45(53.6)$ & 68.4 \\
\hline & 4 & 45 & $4(8.9) ; 41(91.1)$ & $43(51.2) ; 41(48.8)$ & 75.4 \\
\hline & 5 & 41 & $4(9.8) ; 37(90.2)$ & $47(56.0) ; 37(44.0)$ & 82.5 \\
\hline & 6 & 37 & $3(8.1) ; 34(91.9)$ & $50(59.5) ; 34(40.5)$ & 87.7 \\
\hline & 7 & 34 & $4(11.8) ; 30(88.2)$ & $54(64.3) ; 30(35.7)$ & 94.7 \\
\hline & 8 & 30 & $1(3.3) ; 29(96.7)$ & $55(65.5) ; 29(34.5)$ & 96.5 \\
\hline & 9 & 29 & $1(3.5) ; 28(96.6)$ & $56(66.7) ; 28(33.3)$ & 98.2 \\
\hline & 10 & 28 & $1(3.6) ; 27(96.4)$ & $57(67.9) ; 27(32.1)$ & $N A^{* * *}$ \\
\hline \multirow{10}{*}{$\begin{array}{l}\text { Thick liquid } \\
\text { (10 cc) }\end{array}$} & 1 & 66 & 11(16.7); 55(83.3) & 11(16.7); 55(83.3) & 39.3 \\
\hline & 2 & 55 & $7(12.7) ; 48(87.3)$ & 18(27.3): 48(72.7) & 64.3 \\
\hline & 3 & 48 & $5(10.4) ; 43(89.6)$ & $23(34.8) ; 43(65.2)$ & 82.1 \\
\hline & 4 & 43 & $2(4.7) ; 41(95.3)$ & 25(37.9); 41(62.1) & 89.3 \\
\hline & 5 & 41 & $1(2.4) ; 40(97.6)$ & $26(39.4) ; 40(60.6)$ & 92.9 \\
\hline & 6 & 40 & $0(0) ; 40(100.0)$ & $26(39.4) ; 40(60.6)$ & 92.9 \\
\hline & 7 & 40 & $0(0) ; 40(100.0)$ & $26(39.4) ; 40(60.6)$ & 92.9 \\
\hline & 8 & 40 & $0(0) ; 40(100.0)$ & $26(39.4) ; 40(60.6)$ & 92.9 \\
\hline & 9 & 40 & $0(0) ; 40(100.0)$ & 26(39.4); 40(60.6) & 92.9 \\
\hline & 10 & 40 & $2(5.0) ; 38(95.0)$ & 28(42.4): 38(57.6) & $\mathrm{NA} * * *$ \\
\hline
\end{tabular}


To determine the $\mathrm{Se}^{\prime}$ of the FEES protocol for the detection of aspiration, ten swallow trials formed an arbitrary 'gold standard' for determining the likelihood of aspiration. Se' was calculated using the 'gold standard': aspiration (present or absent) at the tenth swallow trial. This procedure was used for both consistencies independently. Overall, the $\mathrm{Se}^{\prime}$ increased along with the number of trials. The $\mathrm{Se}^{\prime}$ of using one trial of thin versus thick consistency was $43.9 \%$ and $39.3 \%$, respectively. By the ninth trial it had risen to $98.2 \%$ for a thin liquid bolus and $92.9 \%$ for a thick one (Table 6.2).

Figures 6.1 and 6.2 show the cumulative percentage of patients with aspiration as a function of the number of swallow trials for thin and thick liquid boluses. The cumulative percentage of patients who aspirated at any of the ten swallow trials with thin liquid is $67.9 \%$ (Figure 6.1, Table 6.2). Using thick liquid, that cumulative percentage is $42.4 \%$ (Figure 6.2, Table 6.2).

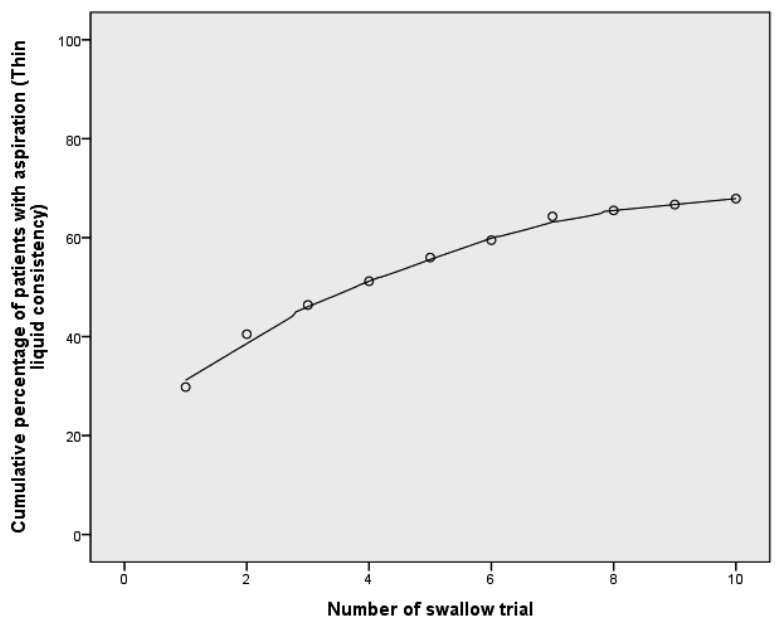

Figure 6.1 Cumulative percentage of patients with thin liquid aspiration, per swallow trial for the total group $(N=84)$.

Next, Kaplan-Meier survival analysis techniques were used to determine the probability of aspiration as a function of the number of swallow trials for each consistency and for each diagnostic group. Figure 6.3 shows the Kaplan-Meier curves using thin liquid for each group separately: patients with oncological disorders $(\mathrm{N}=34)$ and patients with neurological disorders $(\mathrm{N}=50)$. The estimated probability of aspiration using a protocol of one, three, or ten swallow trials is $46 \%, 64 \%$, or $79 \%$ in the oncology group compared to $17 \%, 34 \%$, or $57 \%$ in the neurological group. Figure 6.4 presents results using the same techniques but now for thick consistency. It shows the Kaplan-Meier curves using thick liquid for each group separately: patients with oncological disorders $(\mathrm{N}=26)$ and patients with neurological disorders $(\mathrm{N}=40)$. The estimated probability of aspiration using a protocol of one, three, or ten swallow trials is $27 \%, 47 \%$, or $52 \%$ in the oncological group compared to $8 \%, 25 \%$, or $36 \%$ in the neurological group. None of the patients was 
censored due to a 'competing risk event', which may preclude the event of interest (aspiration) or modify the probability of its onset ${ }^{13}$. To detect differences in the median number of swallow trials necessary to reveal aspiration, data were tested for significant differences between oncological patients and neurological patients using the Log Rank/Mantel-Cox test. Significant group differences were found regarding the median number of swallow trials necessary to reveal aspiration for thin liquid. The oncology and neurology patients differed significantly in this regard (median values 2 and 7 , respectively, $P=0.006$ ), though not for thick liquid (median values 4 and 10 , respectively, $P=0.123)$.

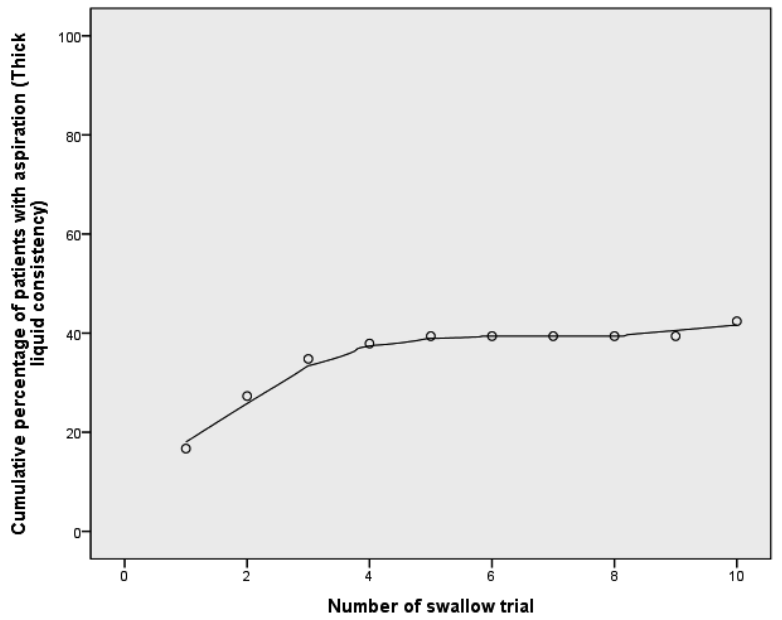

Figure 6.2 Cumulative percentage of patients with thick liquid aspiration, per swallow trial for the total group $(\mathrm{N}=66)$.

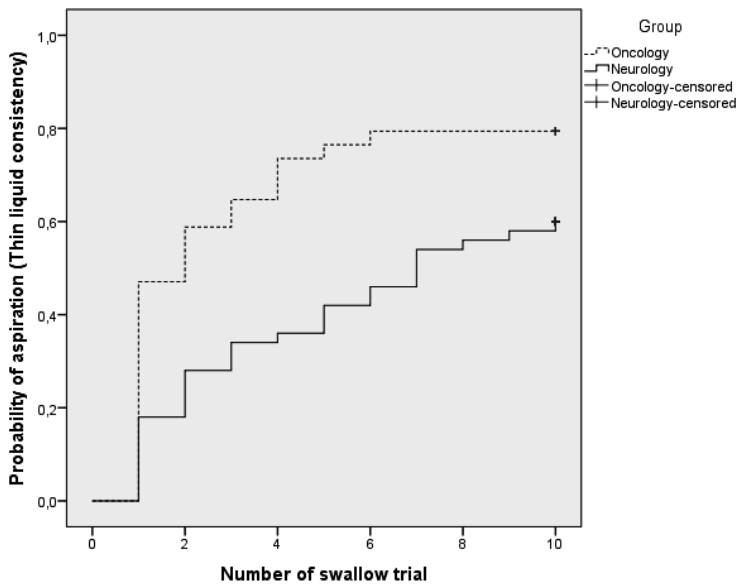

Figure 6.3 Kaplan-Meier survival analysis for the oncological group $(\mathrm{N}=34)$ and neurological group ( $N=50)$ : Probability of aspiration as a function of the number of swallow trials using thin liquid. 


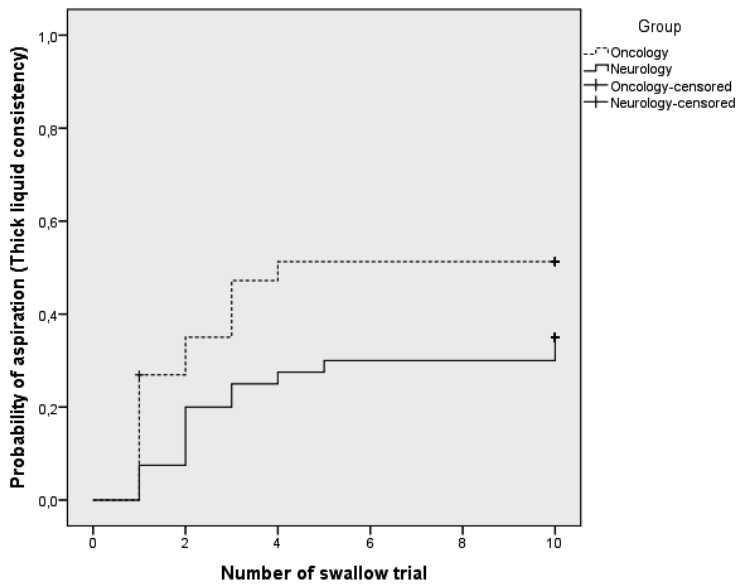

Figure 6.4 Kaplan-Meier survival analysis for the oncological group $(\mathrm{N}=26)$ and neurological group $(\mathrm{N}=40)$ : Probability of aspiration as a function of the number of swallow trials using thick liquid.

\section{Discussion}

\section{New findings}

These preliminary results suggest that dysphagic patients who are at risk for aspiration will not always be identified as such when using a FEES protocol with few (just three or four) swallow trials. After three trials (thin consistency), 39 (46.4\%) patients were identified as aspirators and $45(53.6 \%)$ as non-aspirators (Table 6.2, column 5). At the tenth trial, however, 57 (67.9\%) patients were identified as aspirators and 27 (32.1\%) as nonaspirators. During a FEES in clinical practice, an attempt is made to ascertain the risk of aspiration during daily oral intake. However, no validated FEES protocol exists that recommends a minimum number of swallow trials or order for presenting consistencies to ensure a reliable estimate. Therefore, a tenth swallow trial was adopted as the 'gold standard' to determine the $\mathrm{Se}^{\prime}$ of various protocols. The $\mathrm{Se}^{\prime}$ for detecting aspiration of thin fluids as a function of the number of swallow trials in the total patient group was between 43.9\% (first trial) and $98.2 \%$ (ninth). The $\mathrm{Se}^{\prime}$ for detecting aspiration of thick fluids as a function of the number of swallow trials was between $39.3 \%$ (first trial) and $92.9 \%$ (ninth). High sensitivity is a desirable quality in an assessment tool. These results suggest that a high proportion of true positives (i.e., aspirators) are correctly identifiable after ten swallow trials using the present FEES protocol. The Kaplan-Meier techniques revealed a higher probability of aspiration as a function of the number of trials (for both consistencies) among oncological patients compared with neurological patients (Figures 6.3 and 6.4). However, comparing the number of swallow trials necessary to detect aspiration for thin liquid, the oncology and neurology patients proved to differ 
significantly (median values 2 and 7 , respectively, $P=0.006$ ). Despite the high $\mathrm{Se}^{\prime}$ for detecting aspiration using ten swallow trials, aspiration will not have occurred in all patients by the tenth one (Table 6.2, column 4). For these patients, survival time is said to be censored; if and when a patient will experience aspiration in the future is unclear at the tenth trial. In daily practice, however, patients will probably undergo a FEES consisting of three or four swallow trials. In light of the preliminary data, such protocols may increase the risk of identifying potential aspirators as non-aspirators. Whether FEES can rightly be called the gold standard for detecting aspiration in dysphagic patients depends on which protocol is applied. If a cut-off value of $70 \% \mathrm{Se}^{\prime}$ is set, the number of swallow trials can be limited to three or four, as demonstrated here. However, if assigning a particular protocol the status of gold standard is supposed to ensure that every aspirator is identified ( $\mathrm{Se}^{\prime}$ $100 \%)$, then the number of swallow trials it requires should be increased drastically.

\section{Comparison with other studies}

Most FEES protocols contain fewer swallow trials than the one discussed here $e^{9,14,15,16}$. The present prospective study was designed to estimate the probability of aspiration as a function of the number of swallow trials using a standardized FEES protocol of ten consecutive swallow trials of $10 \mathrm{cc}$ each for two different consistencies (thin and thick liquid) in a group of dysphagic patients. At this moment there is no literature on validated FEES protocols that require a minimum number of swallow trials or order of consistencies to make a reliable estimate of the probability of aspiration. Future research will need to focus on the effects of changing consistencies and volumes in relation to the sensitivity of different FEES protocols. Ideally, similar studies may be conducted to gain deeper insight in the application of videofluoroscopy of swallowing to detect aspiration in patients at risk for oropharyngeal dysphagia. Of the whole range of assessment tools, both FEES and videofluoroscopy are taken as the standard reference for identifying patients at risk for aspiration. Furthermore despite the debate regarding which is a gold standard, FEES was shown to produce more aspiration observations than videofluoroscopy, in concurrently recorded swallows (both FEES and videofluoroscopy simultaneously recorded), arguably overestimating it ${ }^{4}$. For neither standard, however, does the literature offer evidence that any particular protocol is the most sensitive to detect aspiration.

\section{Strength of the study}

The strength of the present study lies in the methodological quality of the whole research design, with its good external and internal validity and therefore its low risk of bias. External validity refers to the process of generalization, specifically whether results obtained from a small sample can be extended to make predictions about the entire population of patients visiting the outpatient clinic for dysphagia. Internal validity refers to an inductive estimate of the degree to which conclusions can be drawn (e.g., cause and effect) based on the measures used and the whole research design ${ }^{17}$. 


\section{Limitations of the study}

The present prospective study has some limitations with respect to methodology and study design. A cross-over study design regarding the presentation of the bolus consistency (thin or thick liquid) may have prevented an order effect if present. Still, the order of the consistencies, thin liquid boluses followed by thick liquid boluses, was based on protocols of daily clinical practice and previous scientific studies ${ }^{6,9}$. The raters, however, scored the blocks of FEES videos (ten swallow acts per consistency) in randomized order.

\section{Clinical applicability of the study}

FEES is a valuable tool for determining aspiration risk. But as the data presented here suggest, FEES protocols with a limited number of swallow trials can underestimate that risk for certain patients. The probability of detecting aspiration using FEES will vary depending on the applied protocol (regarding consistencies offered, number of swallow trials, etc.). To qualify as a gold standard, a FEES protocol would have to identify every aspirator (sensitivity 100\%); to do so, the number of swallow trials should be increased drastically. Whether or not a particular FEES protocol is sensitive enough to be taken as a gold standard is therefore highly dependent on the number of swallow trials offered to the patient. Further research on this matter is recommended. 


\section{References}

1. Martino R, Foley N, Bhogal S, Diamant N, Speechley M, Teasell R. Dysphagia after stroke: incidence, diagnosis, and pulmonary complications. Stroke. 2005;36:2756-2763.

2. Marik PE, Kaplan D. Aspiration Pneumonia and dysphagia in the elderly. Chest 2003;124, 328-336.

3. Rosenbek JC, Robbins JA, Roecker EB, Coyle JL, Wood JL. A penetration-aspiration scale. Dysphagia. 1996;11,93-98.

4. Kelly AM, Drinnan MJ, Leslie P. Assessing Penetration and Aspiration: How Do Videofluoroscopy and Fiberoptic Endoscopic Evaluation of Swallowing Compare? Laryngoscope. 2007;117:1723-1727.

5. Langmore SE, Aviv JE. Endoscopic evaluation and treatment of swallowing disorders. New York: Thieme; 2001.

6. Baijens LWJ. Multidisciplinaire polikliniek voor dysfagie. Nederlands Tijdschrift voor Keel-NeusOorheelkunde. $18^{\mathrm{e}}$ jaargang nummer 1 januari 2012:5-6.

7. Folstein MF, Folstein SE, McHugh PR. "Mini-mental state". A practical method for grading the cognitive state of patients for the clinician. J Psychiatr Res. 1975;12, 189-198.

8. Crary MA, Carnaby Mann GD, Groher ME. Initial psychometric assessment of a Functional Oral Intake Scale for dysphagia in stroke patients. Arch Phys Med Rehabil. 2005;86, 1516-1520

9. Baijens LW, Speyer R, Passos VL, Pilz W, van der Kruis J, Haarmans S, Desjardins-Rombouts C. Surface electrical stimulation in dysphagic parkinson patients: A randomized clinical trial. Laryngoscope. $2013 \mathrm{Apr}$ 17. doi: 10.1002/lary.24119. [Epub ahead of print].

10. Bours GJ, Speyer R, Lemmens J, Limburg M, de Wit R. Bedside screening tests vs. videofluoroscopy or fibreoptic endoscopic evaluation of swallowing to detect dysphagia in patients with neurological disorders: systematic review. J Adv Nurs. 2009;65:477-93.

11. Speyer R, Baijens L, Heijnen M, Zwijnenberg I. Effects of therapy in oropharyngeal dysphagia by speech and language therapists: a systematic review. Dysphagia. 2010;25:40-65.

12. Last JM. A dictionary of epidemiology. Oxford: Oxford University Press; 2001.

13. Jager KJ, van Dijk PC, Zoccali C, Dekker FW. The analysis of survival data: the Kaplan-Meier method. Kidney International. 2008;74:560-565.

14. Dziewas R, Warnecke T, Ölenberg S, Teismann I, Zimmermann J, Krämer C, Ritter M, Ringelstein EB, Schäbitz WR. Towards a Basic Endoscopic Assessment of Swallowing in Acute Stroke - Development and Evaluation of a Simple Dysphagia Score. Cerebrovasc Dis. 2008;26:41-47.

15. Warnecke T, Teismann I, Meimann W, Ölenberg S, Zimmermann J, Krämer C, Ringelstein EB, Schäbitz WR, Dziewas R. Assessment of aspiration risk in acute ischaemic stroke-evaluation of the simple swallowing provocation test. J Neurol Neurosurg Psychiatry. 2008;79:312-314.

16. Leder SB, Espinosa JF. Aspiration risk after acute stroke: comparison of clinical examination and fiberoptic endoscopic evaluation of swallowing. Dysphagia. 2002;17:214-218.

17. Baijens L, Barikroo A, Pilz W. Intrarater and interrater reliability for measurements in videofluoroscopy of swallowing. Eur J Radiol. 2013;82:1683-1695. 
Chapter 7

Summary, general discussion, and future perspectives
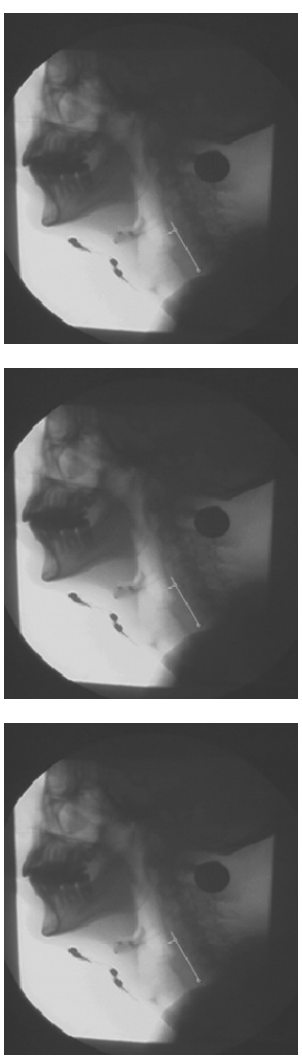
Chapter 7 
The research presented in this thesis had a two-pronged objective: to evaluate surface electrical stimulation of the neck as a new treatment for oropharyngeal dysphagia in idiopathic Parkinson's disease (IPD); and to evaluate observer reliability for measurements made with 'gold standard' assessment tools, namely videofluoroscopy (VFS) and fiberoptic endoscopic evaluation of swallowing (FEES). This new treatment was being applied in clinical practice, especially in the USA, without firm evidence of its therapy effects at the time our research was started. Till then, only one scientific study on the treatment had been published ${ }^{1}$, and it had serious methodological shortcomings. Therefore, we conducted a prospective quasi-randomized controlled trial in a population of 109 patients suffering from oropharyngeal dysphagia and IPD. A detailed discussion per study of this experiment is found in the respective chapters.

\section{Evaluation of treatment techniques}

Before starting the experiment with surface electrical stimulation of the neck, we carried out a systematic review of the literature to evaluate the effect of therapies for oropharyngeal dysphagia in IPD. As asserted in the introduction to this thesis, several techniques are currently applied even though evidence of their effects in this patient population is scarce. This point was confirmed by the results of the literature review, which is described in Chapter 2. Studies were identified by a comprehensive electronic database search using PubMed and Embase. The review covers rehabilitative, surgical, pharmacologic, and other treatments for oropharyngeal dysphagia. The body of literature concerning dysphagia treatment in IPD is small: sixteen articles were included and analyzed. For each one, the critical appraisal items were summarized. Clinical trends in the treatment of oropharyngeal dysphagia in IPD could not be detected. Most studies on therapy effects turn out to have methodological problems. Crucially, their conclusions cannot be compared because the studies applied different therapy methods and outcome measures. Further research that is based on randomized controlled trials would be needed to determine the effectiveness of the various therapies for oropharyngeal dysphagia in IPD.

\section{Pathophysiology of swallowing in IPD}

To better understand the pathophysiology of swallowing in IPD before starting the surface electrical stimulation experiment, we performed a second study using VFS. Chapter 3 discusses the pathophysiological aspects of oropharyngeal swallowing in patients with IPD in detail. VFS was used in a pilot study of the qualitative and quantitative variables of swallowing. A standardized VFS protocol was applied to ten patients with a diagnosis of IPD having dysphagic complaints and to ten healthy age- and gender-matched control 
subjects. Information on their swallowing function was derived from temporal, spatial, and ordinal visuoperceptual variables. After calculating intra- and interobserver reliability, only those variables with sufficient observer reliability were included in the analysis. Interestingly, almost no significant differences were found between IPD patients and healthy control subjects. Apparently, the swallowing function was preserved in the early stages of IPD. Mainly for logistic reasons (e.g., not being able to sit upright for VFS, being too weak for repeated transport to the outpatient clinic, suffering from Parkinson dementia), this study could not enroll many patients with severe IPD, who are often admitted to nursing homes. Nonetheless, the study population was a realistic representation of Parkinson patients consulting the outpatient clinic for dysphagic complaints. Furthermore, as the observer reliability of many swallowing variables proved insufficient, questions were raised about the interpretation of the outcomes of VFS studies as reported in the literature.

\section{Evaluation of surface electrical stimulation of the neck in dysphagic IPD patients}

Following the systematic review of therapy effects and after conducting the study on swallowing pathophysiology in IPD, a new treatment for oropharyngeal dysphagia in IPD was investigated. Chapter 4 describes the effects of a single session of surface electrical stimulation of the neck using different electrode positions in ten patients with IPD and oropharyngeal dysphagia compared with ten age- and gender-matched healthy control subjects during a standardized VFS examination. Three different electrode positions on the neck were applied in random order per subject. For each electrode position, the current was turned "on" or "off" in random order. Temporal, spatial, and ordinal visuoperceptual variables were scored by experienced raters who were blinded to the group, electrode position, and status of the electrical current (on/off). Intra- and interobserver reliability for the VFS measurements were calculated. Few significant effects were observed in dysphagic IPD patients after a single session using different electrode positions. In both groups (IPD patients vs. healthy control subjects), however, significant results for temporal and spatial variables were found when the current was "off", suggesting placebo effects. In that light, further studies are necessary to evaluate the potential therapeutic effect and mechanism of surface electrical stimulation of the neck in dysphagic patients with IPD.

Next, a more elaborate experiment was performed to evaluate the therapy effect. Chapter 5 describes the effects of surface electrical stimulation of the neck (submental region) as adjunct to traditional logopedic dysphagia treatment in dysphagic IPD patients $(N=109)$ in a quasi-randomized controlled trial. Three treatment groups consisting of patients with IPD and oropharyngeal dysphagia were treated daily (30 minutes) for fifteen days with periods of no treatment during the weekend. All three groups received traditional logopedic dysphagia treatment, consisting of the following interventions: 
diverse airway-protecting maneuvers; postural compensation maneuvers; bolus modification and oral intake of various foods; swallowing saliva; and oral motor exercises. Logopedic dysphagia treatment also included rehabilitative techniques geared to improving or restoring the swallowing physiology and facilitating dietary oral intake. It consisted of exercises to improve sensorimotor integration and muscle strength but also involved postural maneuvers. Besides this treatment, two groups received surface electrical stimulation, either motor-level or sensory-level. The subjects were allocated quasi-randomly when the patients were assigned to the treatment groups. The raters were blinded for each other's ratings, the treatment group, and the moment of measurement (pre- or posttreatment). Using proportional odds models, some of the ordinal visuoperceptual outcome variables for measurements in VFS and FEES showed significant improvement in all groups following treatment. No group differences due to surface electrical stimulation of the submental region were noted. Few significant effects were found after undergoing treatment for fifteen days. This suggests a therapy effect of traditional logopedic dysphagia treatment without any additional influence of surface electrical stimulation of the submental region. Several reasons for the absence of therapy effects of the new treatment may be considered: the imprecisely targeted electrical stimulation of tissues with this device; the stimulation of antagonistic muscles involved in swallowing; the fixed stimulation parameters of the electrical stimulation device; the duration of treatment (fifteen days), etc.

\section{Evaluation of FEES protocol}

FEES is one of the instrumental tools that were applied in this study to evaluate therapy effects. FEES is well tolerated, easily repeatable, and is considered a 'gold standard' tool for assessing swallowing physiology. However, various protocols exist, and there is no consensus on the number of swallow trials, bolus consistencies, or bolus volumes to include in a FEES. Chapter 6 reports a prospective study designed to predict the probability of aspiration as a function of the number of swallow trials in dysphagic patients using a standardized FEES protocol of ten consecutive swallow trials of $10 \mathrm{cc}$ each for two different consistencies (thin and thick liquid) in oncological and neurological patients. Eighty-four dysphagic patients were included. One group consisted of patients having oropharyngeal dysphagia due to head and neck cancer and possible oncological treatment effects on swallowing $(\mathrm{N}=34)$. Patients in the other group had oropharyngeal dysphagia as a result of a neurological disease $(\mathrm{N}=50)$. The raters were blinded as to the group (oncology versus neurology). Descriptive statistics, Kaplan-Meier survival analysis technique, and Log Rank/Mantel-Cox tests were used. A hypothetical criterion for the likelihood of aspiration was set arbitrarily at the $10^{\text {th }}$ trial. If no aspiration had occurred during any of the ten consecutive swallows (per consistency), the patient was declared a non-aspirator. The preliminary data suggest that FEES protocols using a limited number of swallow trials can underestimate the aspiration risk in oncological as well as neurological 
patients suffering from oropharyngeal dysphagia, especially when using thin liquid consistencies. Future research should focus on the effects of adjusting consistencies and volumes in relation to the sensitivity of different FEES protocols.

\section{Conclusions and future perspectives}

This thesis offers new and clinically relevant insights into surface electrical stimulation of the neck for oropharyngeal dysphagia in patients with IPD. It demonstrates that surface electrical stimulation had no significant therapy effect in the methodological designs studied for this thesis. Obviously surface electrical stimulation of the neck does not improve the pathophysiological aspects of swallowing in IPD. These observations imply a need to carefully consider whether or not to use surface electrical stimulation for oropharyngeal dysphagia in IPD in clinical practice. Despite the lack of scientific evidence of therapy effects for IPD, the application of surface electrical stimulation of the neck seems to be common practice in the treatment of dysphagic patients. Although no sideeffects emerged during the experiments conducted for this thesis, the possibility of their occurrence should not be disregarded when deciding to treat this patient population with surface electrical stimulation. Further research should focus on several remaining gaps in our knowledge: the values of stimulation parameters; different stimulation sites; simultaneous EMG feedback during surface electrical stimulation of the neck; and choices about the length of a treatment period, the number of sessions, and their duration.

Furthermore, the exact role of traditional logopedic dysphagia treatment in IPD should be specified. This thesis reports a positive therapy effect of traditional logopedic dysphagia treatment. However, its exact content, frequency, and duration remain to be studied in detail so as to compile a clinical decision-making model for treatment options in this patient population. Much work still needs to be done to improve the management of oropharyngeal dysphagia in patients with IPD.

The other focal point in this thesis is the intra- and interobserver reliability of measurements in VFS and FEES. These instrumental assessment tools are considered the 'gold standard' in the evaluation of swallowing pathophysiology and the effects of dysphagia treatment. The authors have determined the intra- and interobserver reliability of a variety of VFS and FEES variables drawn from the literature. The observer reliability of measurements frequently proved to be insufficient, leading to bias in many studies. Therefore, future research should take the issue of observer reliability into account. Moreover, the complementary benefits of using ordinal visuoperceptual and continuous (temporal and spatial) variables in VFS have to be studied more thoroughly. 


\section{References}

1. Freed ML, Freed L, Chatburn RL, Christian M. Electrical stimulation for swallowing disorders caused by stroke. Respir Care. 2001;46:466-474. 

Samenvatting
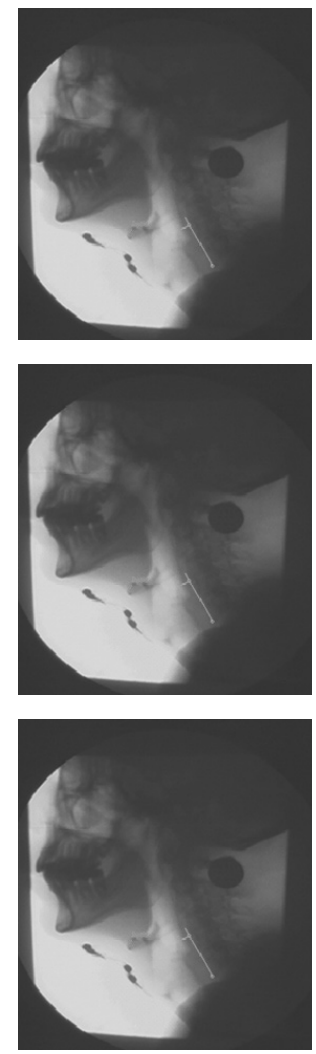
Het onderzoek dat in dit proefschrift wordt beschreven heeft een tweeledige doelstelling: de evaluatie van oppervlakte elektrostimulatie therapie van de hals als een nieuwe behandeling voor orofaryngeale dysfagie bij patiënten met idiopathische ziekte van Parkinson; en de evaluatie van de beoordelaar betrouwbaarheid voor metingen gemaakt met 'gouden standaard' meetinstrumenten zijnde videofluoroscopie en endoscopie van de slikact (FEES: fiberoptic endoscopic evaluation of swallowing). Deze nieuwe behandeling werd toegepast in de klinische praktijk, vooral in de Verenigde Staten, zonder voldoende wetenschappelijk bewijs op het moment dat gestart werd met het onderzoek voor dit proefschrift. Er werd destijds maar één wetenschappelijke studie over deze behandeling gepubliceerd en deze heeft belangrijke methodologische tekortkomingen. Om deze reden hebben wij een prospectieve quasi-gerandomiseerde gecontroleerde trial uitgevoerd in een populatie van 109 patiënten met orofaryngeale dysfagie en idiopathische ziekte van Parkinson. Voor een gedetailleerde discussie per studie wordt $u$ verwezen naar het betreffende hoofdstuk.

\section{Evaluatie van de behandeltechnieken}

Voor aanvang van het experiment met oppervlakte elektrostimulatie van de hals, werd een systematisch literatuuronderzoek verricht om het effect van verschillende behandelingen voor orofaryngeale dysfagie bij idiopathische ziekte van Parkinson te beoordelen. Zoals in de introductie, hoofdstuk 1, van dit proefschrift werd beschreven, worden momenteel verschillende behandeltechnieken toegepast alhoewel het wetenschappelijk bewijs in deze patiëntenpopulatie schaars is. Dit werd bevestigd door de resultaten van het systematisch literatuuronderzoek beschreven in hoofdstuk 2. Studies werden geïdentificeerd door middel van een uitgebreide elektronische databank zoekactie in PubMed en Embase. Dit literatuuronderzoek omvat studies betreffende logopedische revalidatie, chirurgische-, farmacologische- en overige behandelingen voor orofaryngeale dysfagie. De hoeveelheid literatuur over de behandeling van dysfagie in geval van idiopathische ziekte van Parkinson is erg beperkt: zestien artikelen werden geïncludeerd en geanalyseerd. Voor elke studie werden de kritische evaluatie items samengevat. Klinische trends in de behandeling van orofaryngeale dysfagie bij idiopathische ziekte van Parkinson konden niet worden gevonden. De meeste studies over therapie effecten blijken methodologische problemen te hebben. Een cruciaal punt is dat de conclusies niet kunnen worden vergeleken omdat de studies verschillende behandelmethoden en uitkomstmaten gebruiken. Verder onderzoek gebaseerd op gerandomiseerde gecontroleerde trials is nodig om de effectiviteit van de diverse therapieën voor orofaryngeale dysfagie bij idiopathische ziekte van Parkinson na te gaan. 


\section{Pathofysiologie van de slikfunctie bij idiopathische ziekte van Parkinson}

We hebben een tweede studie verricht gebruik makende van videofluoroscopie om een beter inzicht te verkrijgen in de pathofysiologie van de slikact bij idiopathische ziekte van Parkinson voordat het oppervlakte elektrostimulatie experiment werd gestart. Hoofdstuk 3 beschrijft gedetailleerd de pathofysiologische aspecten van de orofaryngeale slikfunctie bij patiënten met idiopathische ziekte van Parkinson. Videofluoroscopie werd gebruikt in deze pilotstudy over kwalitatieve en kwantitatieve variabelen van de slikact. Een gestandaardiseerd videofluoroscopisch protocol werd toegepast op tien patiënten met een diagnose van idiopathische ziekte van Parkinson en dysfagie klachten en tien gezonde leeftijd- en geslacht gematchte controlepersonen. Informatie over hun slikfunctie werd verkregen aan de hand van temporele, spatiële en ordinale visuoperceptieve variabelen. $\mathrm{Na}$ berekening van de intra- en interbeoordelaar betrouwbaarheid werden de variabelen met een voldoende betrouwbaarheid gebruikt in de verdere statistische analysen. $\mathrm{Er}$ werden weinig significante verschillen gevonden tussen de Parkinson patiënten en de gezonde controlepersonen. Blijkbaar is de slikfunctie relatief gespaard in de vroege stadia van idiopathische ziekte van Parkinson. Patiënten met ernstige idiopathische ziekte van Parkinson vaak verblijvend in verpleeghuizen, konden voornamelijk omwille van logistieke redenen (bijvoorbeeld: niet in staat om rechtop te zitten voor videofluoroscopie, te zwak zijn voor herhaald vervoer naar de polikliniek, lijden aan Parkinson dementie, etc.), moeilijker deelnemen aan deze studie. Desalniettemin, vormde de studiepopulatie een realistische weergave van Parkinson patiënten die de polikliniek raadplegen omwille van dysfagie klachten. Bovendien, aangezien de beoordelaar betrouwbaarheid van veel slikfunctie gerelateerde variabelen onvoldoende bleek, ontstonden vragen over de interpretatie van de uitkomsten van videofluoroscopische studies zoals gerapporteerd in de literatuur.

\section{Evaluatie van oppervlakte elektrostimulatie van de hals bij patiënten met idiopathische ziekte van Parkinson en dysfagie}

Naar aanleiding van het systematische literatuuronderzoek naar therapie effecten en na het uitvoeren van de studie over de pathofysiologie van de slikfunctie bij idiopathische ziekte van Parkinson, werd een nieuwe behandeling voor orofaryngeale dysfagie bij idiopathische ziekte van Parkinson onderzocht. Hoofdstuk 4 beschrijft de effecten van een eenmalige sessie van oppervlakte elektrostimulatie van de hals met behulp van verschillende elektrode posities bij tien patiënten met idiopathische ziekte van Parkinson en orofaryngeale dysfagie vergeleken met tien leeftijd- en geslacht gematchte gezonde controlepersonen tijdens een gestandaardiseerd videofluoroscopisch slikonderzoek. Drie verschillende elektrode posities werden toegepast op de hals in willekeurige volgorde per 
subject. Voor elke elektrode positie, werd de stroom 'aan' of 'uit' gezet in willekeurige volgorde. Temporele, spatiële en ordinale visuoperceptieve variabelen werden gescoord door ervaren beoordelaren die geblindeerd waren voor de groep (patiënten versus gezonde controlepersonen), elektrode positie en status van de elektrische stroom (aan/uit). De intra- en interbeoordelaar betrouwbaarheid van de videofluoroscopische metingen werd berekend. Weinig significante effecten werden waargenomen bij Parkinson patiënten met dysfagie na een eenmalige sessie met verschillende elektrode posities. In beide groepen (patiënten versus gezonde controlepersonen) werden echter significante resultaten voor temporele en spatiële variabelen gevonden tijdens stroom 'uit' wat placebo-effecten suggereert. Gezien deze bevindingen zijn verdere studies nodig om de mogelijke therapeutische werking en mechanismen van oppervlakte elektrostimulatie therapie van de hals bij patiënten met idiopathische ziekte van Parkinson en dysfagie te evalueren.

Vervolgens werd een uitgebreider experiment uitgevoerd om het effect van oppervlakte elektrostimulatie therapie van de hals te evalueren. Hoofdstuk 5 beschrijft de effecten van oppervlakte elektrostimulatie therapie van de hals (submentale regio) als aanvulling op traditionele logopedische dysfagie behandeling bij Parkinson patiënten ( $N=109)$ in een quasi-gerandomiseerde gecontroleerde trial. Drie behandelgroepen, bestaande uit patiënten met idiopathische ziekte van Parkinson en orofaryngeale dysfagie werden dagelijks behandeld (30 minuten) gedurende vijftien dagen zonder behandeling tijdens het weekend. Alle drie de groepen kregen traditionele logopedische dysfagie behandeling bestaande uit de volgende interventies: diverse luchtweg beschermende manoeuvres; houdingscompensatie manoeuvres; bolus modificatie en orale inname van verschillende voedingsmiddelen, slikken van speeksel en oraal motorische oefeningen. Logopedische dysfagie behandeling omvatte eveneens revalidatie technieken gericht op het verbeteren of herstellen van de slikfysiologie en faciliteren van orale voedsel inname zoals oefeningen om de sensomotorische integratie en spierkracht te verbeteren, maar ook houdingsmanoeuvres. Naast deze behandeling, kregen twee groepen oppervlakte elektrostimulatie therapie, hetzij motorisch niveau of sensorisch niveau qua stimulatie intensiteit. De proefpersonen werden quasi-gerandomiseerd toegewezen aan de behandelgroepen. De beoordelaren waren geblindeerd voor elkaars scores, de behandelgroep en het moment van de meting (voor of na behandeling). Aan de hand van proportional odds modellen toonden enkele ordinale visuoperceptieve uitkomstvariabelen voor metingen in videofluoroscopie en FEES een significante verbetering in alle groepen na behandeling. $\mathrm{Er}$ werden geen groepsverschillen ten gevolge van oppervlakte elektrostimulatie van de submentale regio geconstateerd. Weinig significante effecten werden gevonden na het ondergaan van oppervlakte elektrostimulatie therapie gedurende vijftien dagen. Dit suggereert een therapie effect van traditionele logopedische dysfagie behandeling zonder bijkomend effect van oppervlakte elektrostimulatie van de submentale regio. Verschillende redenen voor de afwezigheid van therapie effecten van de nieuwe behandeling kunnen worden beschouwd: de onnauwkeurige elektrostimulatie van halsweefsels met dit toestel, het stimuleren van antagonistische spieren die 
betrokken zijn bij de slikfunctie; de vaste stimulatieparameters (fabrieksinstelling) van de elektrostimulator; behandelduur (vijftien dagen), etc.

\section{Evaluatie van het FEES protocol}

FEES is een van de instrumenten die in deze studie werden toegepast om de therapie effecten te evalueren. FEES is een goed te verdragen en gemakkelijk te herhalen onderzoek en wordt beschouwd als een 'gouden standaard' instrument voor de beoordeling van de slikfunctie. Er bestaan echter verschillende uitvoeringsprotocollen en er is geen consensus over het aantal slikacts, bolus consistenties of bolus volumina. Hoofdstuk 6 rapporteert een prospectieve studie ontworpen om de kans op aspiratie als functie van het aantal slikacts te voorspellen in dysfagie patiënten met behulp van een gestandaardiseerd FEES protocol. Het FEES protocol omvatte tien opeenvolgende slikacts van 10 cc elk voor twee verschillende consistenties (dunne en dikke vloeistof). Vierentachtig dysfagie patiënten werden geïncludeerd. Eén groep bestond uit patiënten met orofaryngeale dysfagie ten gevolge van hoofd-hals kanker en mogelijke oncologische behandeleffecten op de slikfunctie $(\mathrm{N}=34)$. Patiënten in de andere groep hadden orofaryngeale dysfagie als gevolg van een neurologische ziekte $(\mathrm{N}=50)$. De beoordelaren waren geblindeerd voor de groep (oncologie versus neurologie). Beschrijvende statistiek, Kaplan-Meier survival analyse techniek en Log Rank / Mantel-Cox testen werden gebruikt. Een hypothetisch criterium voor de kans op aspiratie is arbitrair gezet op de 10e slikact. Indien tijdens een van de tien opeenvolgende slikacts (per consistentie) geen aspiratie had plaatsgevonden, werd de patiënt geklasseerd als een non-aspirator. Deze voorlopige gegevens suggereren dat FEES protocollen met een beperkt aantal slikacts het aspiratie risico kunnen onderschatten in oncologische en neurologische patiënten met orofaryngeale dysfagie, vooral bij gebruik van dun vloeibare consistenties. Toekomstig onderzoek moet zich richten op de effecten van het aanpassen van bolus consistenties en volumina in relatie tot de sensitiviteit van verschillende FEES protocollen.

\section{Conclusies en toekomstperspectieven}

Dit proefschrift biedt nieuwe en klinisch relevante inzichten in oppervlakte elektrostimulatie therapie van de hals voor orofaryngeale dysfagie bij patiënten met idiopathische ziekte van Parkinson. Oppervlakte elektrostimulatie therapie had geen significant effect in de methodologische ontwerpen bestudeerd voor dit proefschrift. Blijkbaar verbeteren de pathofysiologische aspecten van de slikfunctie bij idiopathische ziekte van Parkinson niet door het gebruik van oppervlakte elektrostimulatie van de hals. Deze waarnemingen impliceren dat het noodzakelijk blijft om zorgvuldig te overwegen of oppervlakte elektrostimulatie voor orofaryngeale dysfagie bij idiopathische ziekte van 
Parkinson in de klinische praktijk al dan niet moet worden gebruikt. Ondanks het ontbreken van wetenschappelijk bewijs over therapie effecten bij idiopathische ziekte van Parkinson lijkt de toepassing van oppervlakte elektrostimulatie van de hals gebruikelijk in de behandeling van dysfagie patiënten. Hoewel er geen bijwerkingen werden geobserveerd tijdens de experimenten van dit proefschrift, moet de mogelijkheid van het optreden daarvan niet buiten beschouwing worden gelaten bij de beslissing om deze patiëntenpopulatie met oppervlakte elektrostimulatie te behandelen. Verder onderzoek moet zich richten op een aantal hiaten in onze kennis: de waarden van de stimulatie parameters; verschillende anatomische stimulatie locaties; gelijktijdige EMG feedback tijdens oppervlakte elektrostimulatie van de hals en keuzes over de lengte van een behandelperiode, het aantal sessies en hun duur.

Bovendien moet de precieze rol van traditionele logopedische dysfagie behandeling bij idiopathische ziekte van Parkinson worden gespecificeerd. Dit proefschrift rapporteert een positief therapie effect van traditionele logopedische dysfagie behandeling. Echter, de precieze inhoud, de frequentie en de duur van de behandeling dienen te worden bestudeerd teneinde een klinisch besluitvormingsmodel voor de behandelopties in deze patiëntenpopulatie op te stellen. Er valt nog veel werk te verrichten om het management van orofaryngeale dysfagie bij patiënten met idiopathische ziekte van Parkinson te verbeteren.

Het andere centrale punt in dit proefschrift is de intra- en interbeoordelaar betrouwbaarheid van de metingen in videofluoroscopie en FEES. Deze instrumenten worden beschouwd als de 'gouden standaard' in de evaluatie van de pathofysiologie van de slikfunctie en de effecten van dysfagie behandeling. De auteurs hebben de intra- en interbeoordelaar betrouwbaarheid van verschillende videofluoroscopische en FEES variabelen uit de literatuur bepaald. De beoordelaar betrouwbaarheid van metingen is vaak onvoldoende gebleken, wat aanleiding geeft tot bias in vele studies. Daarom moet toekomstig onderzoek rekening houden met de beoordelaar betrouwbaarheid. Bovendien moeten de complementaire voordelen van het gebruik van ordinale visuoperceptieve en continue (temporele en spatiële) variabelen in videofluoroscopie grondiger worden bestudeerd. 


\section{References}

1. Freed ML, Freed L, Chatburn RL, Christian M. Electrical stimulation for swallowing disorders caused by stroke. Respir Care. 2001;46:466-474. 
Dankwoord
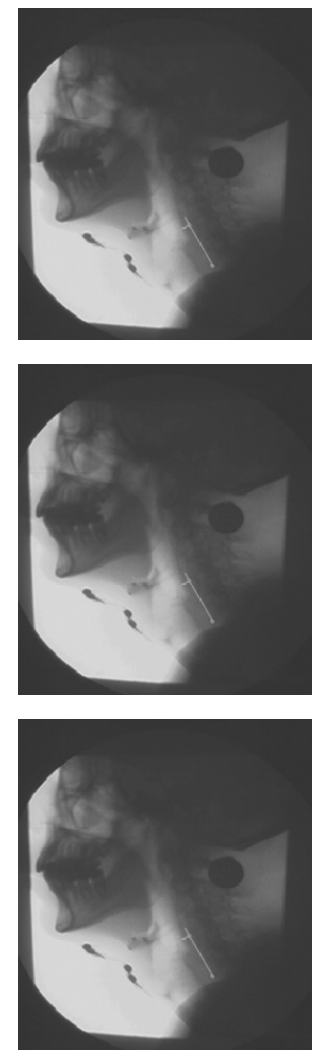


\section{Dankwoord}

Dit proefschrift was niet tot stand gekomen zonder de hulp van veel mensen. Graag wil ik iedereen die aan dit proefschrift heeft bijgedragen hartelijk danken en een aantal mensen in het bijzonder.

Prof. dr. B. Kremer, beste Bernd, dit proefschrift is tot stand gekomen op de afdeling KNO onder jouw leiding. Dank voor het geven van de ruimte om wetenschappelijk onderzoek en klinische activiteiten te combineren. Dank voor jouw vertrouwen in mijn onderzoekslijn.

Dr. R. Speyer, beste Renée, mijn eerste kennismaking met wetenschappelijk onderzoek verliep via jou. Ik heb waardevolle kennis opgedaan onder jouw begeleiding waar ik nog altijd veel aan heb. Ik heb bewondering voor je wetenschappelijke kennis en je mogelijkheden om methodologische knelpunten bij te sturen. Nogmaals heel hartelijk dank voor je hulp.

Mw. W. Pilz, beste Walmari, grote bewondering heb ik voor je niet aflatende vastberadenheid om de data van mijn proefschrift, door middel van legers van studenten die het beeldmateriaal beoordeelden, onder de knie te krijgen. Nu is het jouw beurt om te promoveren en mijn beurt om jou daarbij te helpen.

Mw. N. Roodenburg, beste Nel, hartelijk dank voor alle jaren onvoorwaardelijke hulp en belangstelling voor het dysfagie-onderzoek. Zowel als vriendin en als collega ben ik je de afgelopen jaren enorm gaan waarderen en hoop ik nog jaren met je te mogen samenwerken.

Dr. V. Lima Passos, beste Valéria, hartelijk dank voor jouw statistische bijdrage aan dit proefschrift. Ik heb veel van je geleerd en waardeer jouw belangstelling voor het dysfagieonderzoek.

Prof. dr. J.J. Manni, beste Hans, hartelijk dank om mij een plaats te geven in de staf KNO in Maastricht destijds. Jij hebt mij als eerste gestimuleerd om te promoveren en me geholpen hiervoor de nodige financiële middelen te verkrijgen.

De beoordelingscommissie: Prof. dr. R.J. van Oostenbrugge, Dr. A.F.G. Leentjens, Prof. dr. A.A.M. Masclee, Prof. dr. M.W.M. van den Brekel, hartelijk dank voor de bereidwilligheid om een deskundig oordeel te geven over dit proefschrift.

Alle stafleden en AIOS van de afdeling KNO, dank voor jullie geduld en belangstelling voor mijn onderzoek. Ik waardeer dat ik de ruimte krijg om dysfagie-onderzoek te mogen verrichten. 
Alle medewerkers van de afdeling radiologie, Stem Spraak Taal (SST), stafsecretariaat KNO en poli KNO, dank voor jullie hulp en ondersteuning van mijn werkzaamheden bij de totstandkoming van dit proefschrift.

Mama en papa, dankzij jullie ben ik de goede richting uitgestuurd om te komen waar ik nu ben. Zonder jullie onvoorwaardelijke steun en aandacht had ik dit niet gered.

Willem, Thijs en Lode, jullie aanwezigheid en steun zijn onmisbaar voor mij. Willem, dank voor al je geduld de afgelopen jaren voor wat betreft mijn drang om me bezig te houden met wetenschap en gezondheidszorg. Ik hoop dat ik met jullie oud mag worden. 
Curriculum vitae
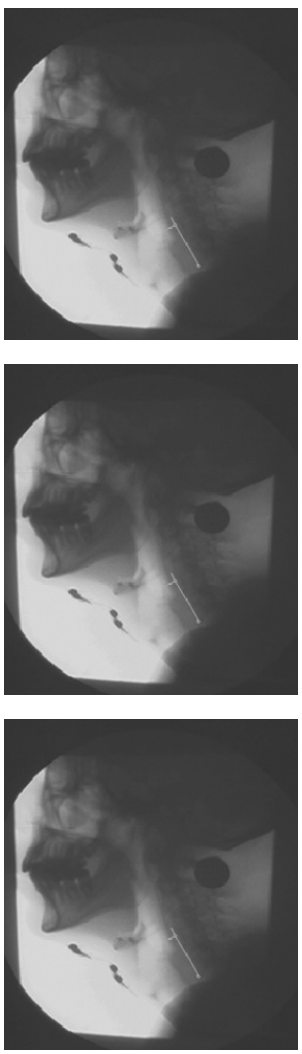


\section{Curriculum vitae}

Laura Baijens is geboren op 21 juni 1972 te Helmond. Vanaf het eerste levensjaar is zij opgegroeid in Waspik. Van 1984 tot en met 1990 heeft zij in Dongen op het Dr. Schaepmancollege het VWO gevolgd. In 1990 werd gestart met de opleiding Geneeskunde aan de Rijksuniversiteit Gent, België, dat tussentijds werd bezegeld met het behalen van de kandidatuur met grote onderscheiding. De opleiding werd in 1997 afgerond met een onderscheiding. Na een korte werkperiode als arts-assistent-niet-in-opleiding in het Henri Serruysziekenhuis te Oostende werd in april 1998 gestart met de opleiding tot Neus-, Keelen Oorarts aan de Rijksuniversiteit Gent en het Universitair Ziekenhuis Gent. Gedurende de opleiding werd een perifere stage gevolgd van 2 jaar in het Algemeen Stedelijk Ziekenhuis te Aalst. Gedurende 1 jaar werd de opleiding vervolgd op de afdeling KNO in het St. Radboud Ziekenhuis (UMCN) te Nijmegen. Per maart 2003 was de opleiding afgerond.

Vanaf juni 2003 is ze KNO-arts/laryngoloog en staflid op de afdeling KNO in het Academisch Ziekenhuis Maastricht (MUMC). Haar belangrijkste aandachtsgebied is orofaryngeale dysfagie (diagnostiek en behandeling). Zij is coördinator van het multidisciplinair spreekuur voor slikstoornissen dat bestaat uit een samenwerkingsverband tussen de afdelingen KNO, logopedie en radiologie sinds 2007. De slikstoornis van patiënten wordt door het multidisciplinair team door middel van een dagbehandeling geanalyseerd waarna een therapeutisch advies volgt. De gestandaardiseerde meetgegevens uit de databank van deze dagbehandeling geven momenteel gevolg aan nieuwe promotieonderzoeken. Zij is regelmatig gevraagd spreker op internationale cursussen en congressen aangaande orofaryngeale dysfagie. Op dit moment bestaan haar taken, naast wetenschappelijke activiteiten, uit patiëntenzorg zoals laryngologie, algemene KNO, neonatale- en kinderluchtwegproblematiek, transsfenoïdale hypofysechirurgie, (benigne) weke delen chirurgie hals, Zenker diverticulotomie en deelname aan diverse (transmurale) multidisciplinaire teams (schisis, OSAS, neurofibromatosis, thuisbeademing-CTB, cystic fibrosis, myotone dystrofie, luchtwegstenosen etc.).

Zij is voorzitter van de richtlijn diagnostiek en behandeling slikstoornissen van de Nederlandse Vereniging voor Keel-Neus-Oorheelkunde en Heelkunde van het HoofdHalsgebied.

Tijdens het promotieonderzoek is zij getrouwd met Willem Leurs en werden Thijs en Lode geboren. Dit proefschrift staat aan het begin van de huidige onderzoekslijn orofaryngeale dysfagie op de afdeling KNO in Maastricht (MUMC). 

List of publications
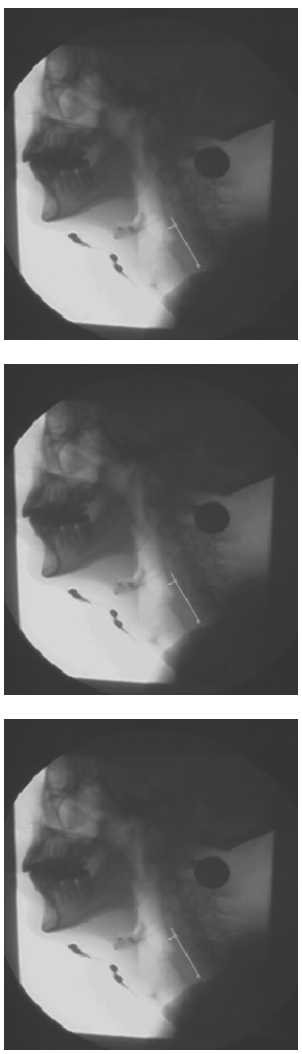


\section{List of publications}

Michou E, Baijens LWJ, Rofes L, Sanz Cartagena P, Clavé P. Oropharyngeal Swallowing Disorders in Parkinson's Disease: revisited. International Journal of Speech \& Language Pathology and Audiology. In press.

Pilz W, Baijens LWJ, Kremer B. Oropharyngeal Dysphagia in Myotonic Dystrophy: a systematic review. Dysphagia. In press.

Verdonschot R, Baijens LWJ, Serroyen JL, Leue C, Kremer B. Symptoms of anxiety and depression assessed with the Hospital Anxiety and Depression Scale in patients with oropharyngeal dysphagia. J Psychosom Res. 2013;75:451-455.

Baijens LWJ, Barikroo A, Pilz W. Intrarater and interrater reliability for measurements in videofluoroscopy of swallowing. Eur J Radiol. 2013;82:1683-1695.

Baijens LWJ, Koetsenruijter K, Pilz W. Diagnosis and treatment of phagophobia: a review. Dysphagia. 2013;28:260-270.

Baijens LWJ, Speyer R, Passos VL, Pilz W, van der Kruis J, Haarmans S, DesjardinsRombouts C. Surface electrical stimulation in dysphagic Parkinson patients: A randomized clinical trial. Laryngoscope. 2013;123:E38-44.

Baijens LWJ, Speyer R, Passos VL, Pilz W, Roodenburg N, Clavé P. The effect of surface electrical stimulation on swallowing in dysphagic Parkinson patients. Dysphagia. 2012;27:528-537.

Heijnen BJ, Speyer R, Baijens LWJ, Bogaardt HC. Neuromuscular electrical stimulation versus traditional therapy in patients with Parkinson's disease and oropharyngeal dysphagia: effects on quality of life. Dysphagia. 2012;27:336-345.

Mendels EJ, Brunings JW, Hamaekers AEW, Stokroos RJ, Kremer B, Baijens LWJ. Incidence of vocal cord injury and hoarseness due to general anesthesia: a systematic review. Arch Otolaryngol Head Neck Surg. 138:257-264, 2012.

Baijens LWJ, Speyer R, Lima Passos V, Pilz W, Roodenburg N, Clavé P. Swallowing in Parkinson patients versus healthy controls: Reliability of measurements in videofluoroscopy. Gastroenterol Res Pract. 2011;2011:380682. 
Speyer R, Heijnen BJ, Baijens LWJ, Vrijenhoef F, Otters E, Roodenburg N, Bogaardt HC. Quality of life in oncological patients with oropharyngeal dysphagia: Validity and reliability of the Dutch version of the Deglutition Handicap Index (DHI) and the MD Anderson Dysphagia Inventory (MDADI). Dysphagia. 2011;26:407-414.

van der Kruis J, Baijens LWJ, Speyer R, Zwijnenberg I. Biomechanical analysis of hyoid bone displacement in videofluoroscopy: a systematic review of intervention effects. Dysphagia. 2011;26:171-182.

Baijens LWJ, Speyer R, Roodenburg N, Hilgers F. Prosthetic tracheoesophageal voice production and swallowing function in a patient following circumferential pharyngolaryngectomy and neopharyngeal reconstruction with a jejunal free flap. Dysphagia. 2011;26:78-84.

Speyer R, Baijens LWJ, Heijnen MAM, Zwijnenberg I. Effects of therapy in oropharyngeal dysphagia by speech and language therapists (a systematic review). Dysphagia. 2010;25: 40-65.

Speyer R, Bogaardt HCA, Lima Passos V, Roodenburg N, Zumach A, Heijnen MAM, Baijens LWJ, Fleskens SJHM, Brunings JW. Maximum phonation time: variability and reliability. J Voice. 2010;24:281-284.

Baijens LWJ, Speyer R. Effects of therapy for dysphagia in Parkinson's disease (a systematic review). Dysphagia. 2009;24:91-102.

Bogaardt HCA, Speyer R, Baijens LWJ, Fokkens WJ. Cross-cultural adaptation and validation of the Dutch version of SWAL-QOL. Dysphagia. 2009;24:66-70.

Bülow M, Speyer R, Baijens LWJ, Woisard V, Ekberg O. Neuromuscular electrical stimulation (NMES) in stroke patients with oral and pharyngeal dysfunction. Dysphagia. 2008;23:302-309.

Baijens LWJ, Speyer R, Roodenburg N, Manni JJ. The Effects of Neuromuscular Electrical Stimulation for Dysphagia in Opercular Syndrome: a case study. Eur Arch Otorhinolaryngol. 2008;265:825-830.

Baijens LWJ, Speyer R, Linssen M, Ceulen R, Manni JJ. Rejection of injectable silicone used for vocal fold augmentation. Case Report. Eur Arch Otorhinolaryngol. 2007;264:565-568.

Baijens LWJ, Manni JJ. Paraneoplastic syndromes in patients with primary malignancies of the head and neck. Four cases and a review of the literature. Eur Arch Otorhinolaryngol. 2006;263:32-36. 
Baijens LWJ, Manni JJ. Isolated malleus handle fracture. Audiol Medicine. 2005;3:121-123.

Baijens LWJ, Deleenheer E, Cremers C. Stickler Syndrome type I and Stapes ankylosis. Int J Ped ORL. 2004;68:1573-1580. 


